\title{
FULL CUSTOM VLSI DESIGN OF ON-LINE STABILITY CHECKERS
}

\author{
A Thesis \\ presented to \\ the Faculty of California Polytechnic State University, \\ San Luis Obispo
}

\author{
In Partial Fulfillment \\ of the Requirements for the Degree \\ Master of Science in Electrical Engineering \\ by \\ Chris Y. Lee
}

August 2011 
(C) 2011

Chris Y Lee

ALL RIGHTS RESERVED 


\section{COMMITTEE MEMBERSHIP}

TITLE:

AUTHOR:

DATE SUBMITTED:

COMMITTEE CHAIR:

COMMITTEE MEMBER:

COMMITTEE MEMBER:
Full Custom VLSI Design of On-line Stability Checkers

Chris Y. Lee

August 2011

John Oliver, Assistant Professor

Dennis Derickson, Professor

Dean Arakaki, Assistant Professor 


\section{ABSTRACT \\ Full Custom VLSI Design of On-line Stability Checkers}

Chris Y. Lee

A stability checker is a clocked storage element, much like a flip-flop, which detects unstable and late signals in the pipeline of a digital system. The On-line stability checker operates concurrently with its associated circuit-under-test (CUT). This thesis describes the full custom very-large-scale integration (VLSI) design and testing process of On-Line Stability Checkers. The goals of this thesis are to construct and test Stability Checker designs, and to create a design template for future class projects in the EE 431 Computer-Aided Design (CAD) of VLSI Devices course at Cal Poly.

A method for concurrent fault testing called On-line Stability Checking was introduced by Franco and McCluskey [10] to detect reliability failures. Reliability failures initially manifest themselves as delay faults and transient glitching, which become progressively larger over time due to the wearout of conducting metal lines, eventually leading to functional faults. Stability checkers periodically detect reliability failures by monitoring CUT output signals for unstable and late input signals over a time period after the sampling clock edge.

The checkers are tested by applying variable delayed input test patterns to emulate reliability failures. Consequently, configurable delay chains were incorporated into the system to provide variable delays on the input signal lines. The system also includes external test signal ports. 
Circuit and layout designs were implemented in the Electric VLSI Design tool [12] and simulated with LTSPICE [13]. Electric provides Design Rule Checking (DRC) and Layout-versus-Schematic (LVS) utilities for verification. Each module was designed in a bottom-up, hierarchical cell-based approach. Functional simulation, DRC and LVS checks were performed at every subsequent higher cell layer in the design hierarchy. The final chip layout was taped out for fabrication on November 29, 2010 and finished parts were received on July 7, 2011 after two manufacturing delays.

Finished packaged parts were successfully verified for functionality based on SPICE simulations. The stability checkers were tested for flip-flop operation, glitch detection and late signal arrival detection. Configurable delay chains were tested to determine delay resolution and uniformity. Actual delay resolution and range measurements show a 3 to 4 times difference compared to simulated values.

The Electric design template created from this project includes basic CMOS logic gates with uniform standard cell heights. The template contains a 40-pin pad ring cell along with the individual pad ring components. EE 431 students would be able to create custom chips that are compatible for fabrication via the MOSIS MEP service. In future work, the template design library can be expanded to include more logic gate variants of various inputs and drive strengths as well as more complex functional modules.

Keywords: application specific integrated circuits, logic design, digital simulation, error analysis, sequential logic circuit fault testing, 


\section{ACKNOWLEDGMENTS}

I owe a great deal to my advisor Dr. John Oliver, who granted me this opportunity to work on this exciting project. I would like to express my sincerest gratitude for his time, effort and mentorship that inspired me throughout this endeavor. The success of this project would not have been possible without his supervision and guidance. I have learned invaluable lessons on getting digital chips from conception into actual physical prototypes.

I would like to thank Dr. Jacob Baker of Boise State University for taking the time to verify my designs during the critical tapeout stage. I also greatly appreciate all the documentation and tutorial videos on chip design that he provided on his homepage. To my thesis committee, Dr. Dennis Derickson and Dr. Dean Arakaki, thank you for your time and advice in helping me refine my work.

Dr. James Harris provided constructive ideas on thesis writing and test planning. I am thankful for his constant support and interest in my work. I would like to acknowledge and thank Jaime Carmo for his assistance in setting up equipment for testing, and also to Dr. Richard Savage who helped arrange my visit to the microfab lab for dice photography.

I would also like to extend my thanks to the staff at MOSIS for facilitating the entire process and for attending to all my inquiries. These people include: Helen Thompson, Wes Hanford, Michael Mekonnen, Robert Wormuth and Sam Reynolds.

Most of all, I would like to thank my family and friends. My success was only possible with all your love and support. 


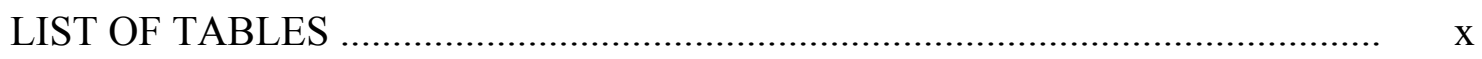

LIST OF FIGURES ……………….......................................................... CHAPTER

$1 \quad$ INTRODUCTION ......................................................................... 1

2 BACKGROUND ...........................................................................

2.1 Stability Checking .......................................................................... 3

2.1.1 Delay Fault Testing ............................................................. 4

2.1.2 Limitations of Delay Fault Testing ……………………….... 5

2.1.3 On-line Stability Checking ……………………………….... 6

2.2 Related Work on On-Line Testing ………………………………..... 8

2.3 Survey on Variable Delay Techniques .............................................. 9

2.3.1 Current Starvation Technique …………………………....... 9

2.3.2 Shunt Capacitor Technique ………………………................ 11

2.3.3 Variable Resistor Technique ………………….................... 12

2.3.4 Comparison of Variable Delay Techniques ………………... 13

$2.4 \quad$ ASIC Fabrication Services ............................................................. 15

3 CHIP DESIGN …........................................................................ 17

3.1 System Specifications …………………………........................ 17

3.1.1 Process Technology ……………………………………....... 18

3.2 Stability Checkers ………………………………...................... 19

3.2.1 Function ........................................................................... 19

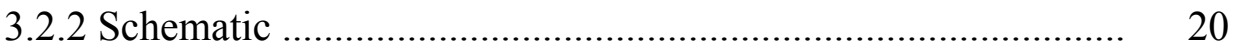

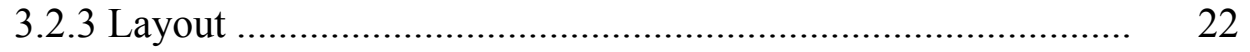

3.2.4 Verification ...................................................................... 24

3.3 Configurable Delay Chain ............................................................ 25

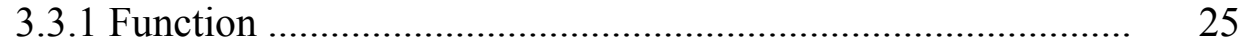

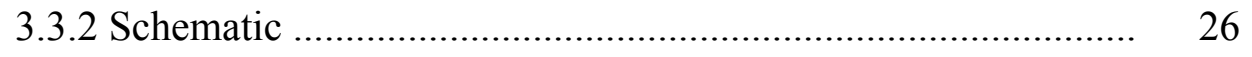

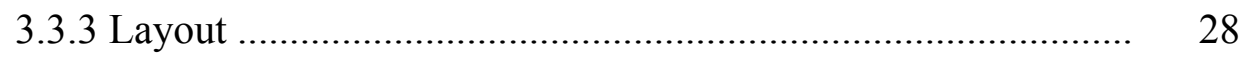


3.3.4 Verification ........................................................................ $\quad 30$

$3.4 \quad$ One-Cold Decoder ………………………………........................ 31

3.4.1 Function ......................................................................... 31

3.4.2 Schematic ..................................................................... 31

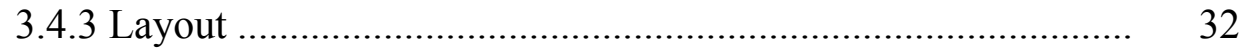

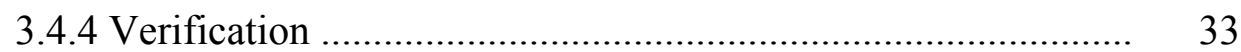

3.5 Full Chip Design ...................................................................... 34

3.5.1 Floorplan ................................................................... 34

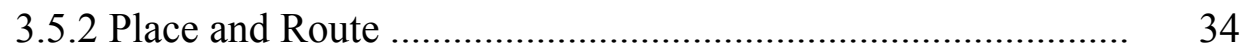

3.5.3 Verification ........................................................................ 37

$4 \quad$ POST-SILICON VERIFICATION …………...................................... 40

4.1 Manufacturing Issues .................................................................. 40

4.2 Visual Inspection ................................................................... 41

$4.3 \quad$ Test Procedures ........................................................................... 44

$4.4 \quad$ Power-up Test ...................................................................... 48

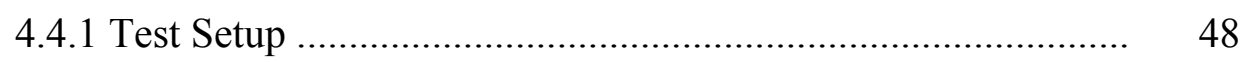

4.4.2 Test Results ........................................................................ 48

4.5 Clock Test .......................................................................... 49

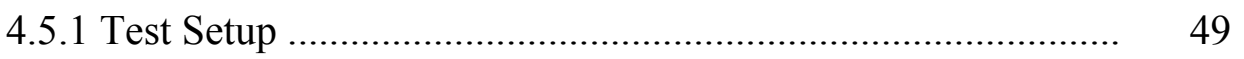

4.5.2 Test Results ................................................................. 50

4.6 Configurable Delay Test ............................................................ 52

4.6.1 Test Setup ......................................................................... 52

4.6.2 Test Results .................................................................... 53

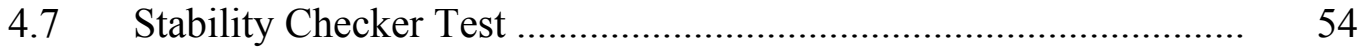

4.7.1 Test Setup ……………………..................................... 54

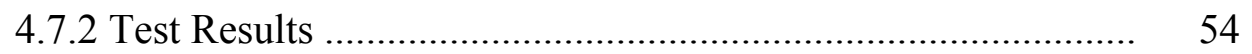

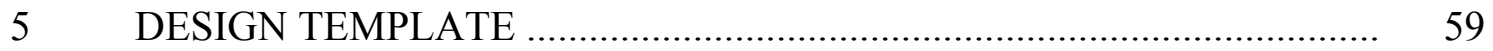

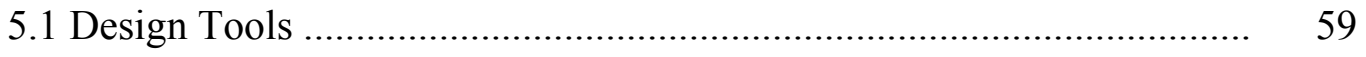

5.2 Template Project .................................................................................. 61

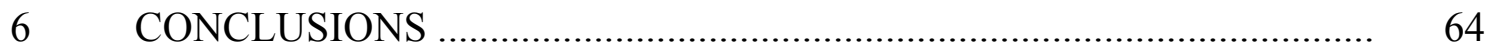

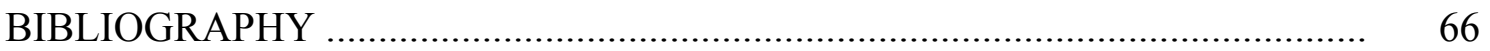


A. Endorsement Letter for MOSIS Subsidy ..................................... 72

B. MOSIS Price Quotation .............................................................. 73

C. MOSIS ON Semi C5 Characterization Data and SPICE models ..... 74

D. Full Chip Routing Layers .................................................... 78

E. SPICE Codes for Pre-Silicon Verification .................................... 81

F. Manufacturability Review Results ............................................. 82

G. Tapeout Confirmation ….......................................................... 83

H. Initial Bonding Diagram .................................................... 84

I. Notification of Fabrication Failure ........................................... 85

J. Notification of Packaging Anomaly ............................................ 86

K. $\quad$ Final Bonding Diagram ............................................................. 87

L. Die Structure Measurements .................................................... 88

M. Chip Pinout ..................................................................... 91

N. $\quad$ SPICE Codes for Post-Silicon Verification .................................... 92

N.1 Configurable Delay Chain .............................................. 92

N.2 Stability Checker Glitch Detection ................................. 94

N.2 Stability Checker Late Signal Detection ........................... 94

O. MOSIS Educational Program ...................................................... 95

O.1 Accounts for Academic Institutions ...................................... 95

O.1.1 Commercial Account ........................................... 95

O.1.2 MEP Account ................................................... 96

O.2 MEP Research Program ..................................................... 97

O.2.1 Setting Up A MOSIS Customer Account ................ 98

O.2.2 Accessing Vendor Documents ............................... 98

O.2.3 Submitting a Purchase Order to MOSIS .................. 100

O.2.4 Submitting a Design to MOSIS ............................ 100 


\section{LIST OF TABLES}

\begin{tabular}{|c|}
\hline 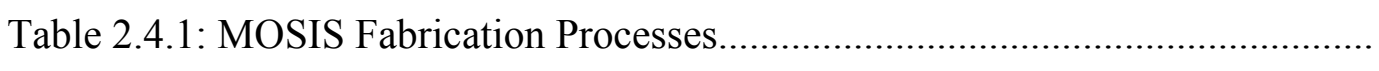 \\
\hline Table 3.2.1: Error Transistor Dimensions ............................... \\
\hline 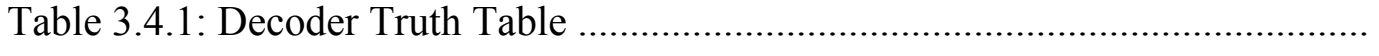 \\
\hline 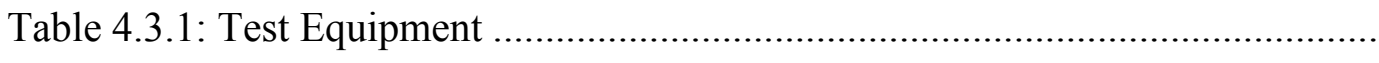 \\
\hline 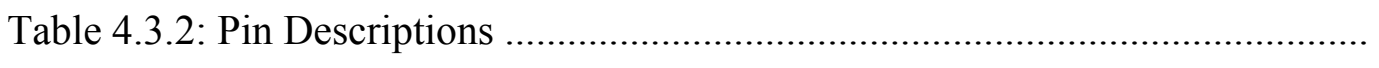 \\
\hline Table 4.4.1: Bus Pin Assignments ....... \\
\hline
\end{tabular}




\section{LIST OF FIGURES}

Page

Fig. 2.1.1 Hardware Model ............................................................................. 4

Fig. 2.1.2 Delay Fault Testing Waveforms ........................................................... 4

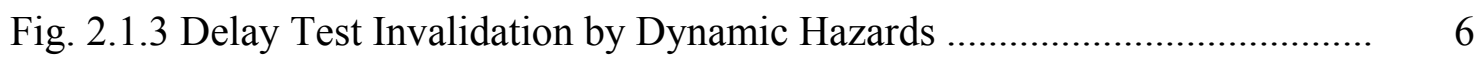

Fig. 2.1.4 Stability Checking Waveforms ........................................................ 7

Fig. 2.3.1 Current Starved Delay Element ............................................................ 10

Fig. 2.3.2 DCDE Architecture ......................................................................... 11

Fig. 2.3.3 Shunt Capacitor Cell …................................................................. 11

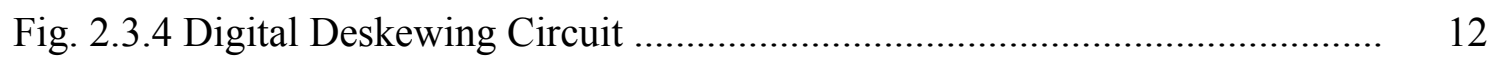

Fig. 2.3.5 Variable Resistor Configurable Delay .................................................... 13

Fig. 2.3.6 Variable Delay SPICE Simulations .................................................. 15

Fig. 3.1.1 System Block Diagram ................................................................. 18

Fig. 3.2.1 Master-Slave Flip-flop Schematic ....................................................... 20

Fig. 3.2.2 Stability Checker Schematic ........................................................... 21

Fig. 3.2.3 Stability Checker Layout ................................................................... 22

Fig. 3.2.4 Stability Checker Variants ............................................................. 23

Fig. 3.2.5 Stability Checker Simulation ............................................................... 24

Fig. 3.3.1 Configurable Delay Schematic ........................................................ 27

Fig. 3.3.2 Configurable Delay Block Diagram ................................................ 28

Fig. 3.3.3 Configurable Delay Chain Layout ....................................................... 29

Fig. 3.3.4 Configurable Delay Simulation ....................................................... 30

Fig. 3.3.5 Simulated Delay Measurements ......................................................... 30

Fig. 3.4.2 Decoder Schematic ........................................................................... 32

Fig. 3.4.3 Decoder Layout ....................................................................... 32

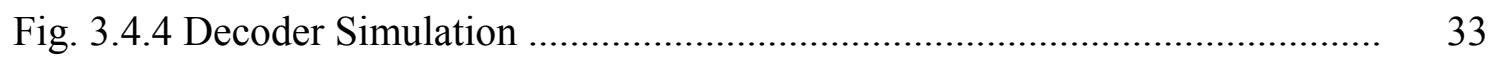

Fig. 3.5.1 Chip Floorplan .............................................................................. 34

Fig. 3.5.2 System Schematic ........................................................................ 35

Fig. 3.5.3 System Layout ............................................................................. 36

Fig. 3.5.4 Full Chip Schematic .................................................................. 36

Fig. 3.5.5 Full Chip Layout ...................................................................... 37 
Fig. 3.5.6 Full Chip Simulation ....................................................................... 38

Fig. 4.2.1 Unpackaged Dice ........................................................................... 41

Fig. 4.2.2 Packaged Chip ................................................................................ 42

Fig. 4.2.3 Die Photograph .......................................................................... 43

Fig. 4.2.4 Wire Bonded Die ...................................................................... 44

Fig. 4.3.1 Block Diagram of Test Setup ............................................................ 45

Fig. 4.3.2 Test Setup ................................................................................... 46

Fig. 4.3.3 Test Chip Setup ............................................................................ 47

Fig. 4.4.1 Power-Up Test Waveforms ………………........................................... 49

Fig. 4.5.1 Clock Simulation Waveforms ………………………….................... 50

Fig. 4.5.2 Chip Clock Test Waveforms ……………........................................ 51

Fig. 4.5.3 Propagation Delay Measurement …………......................................... 51

Fig. 4.6.1 Configurable Delay Waveforms ………………................................. 52

Fig. 4.6.2 Configurable Delay Measurements ........................................................ 53

Fig. 4.7.1 Flip-flop Operation Test Waveforms ..................................................... 54

Fig. 4.7.2 Glitch Test Waveforms ...................................................................... 55

Fig. 4.7.3 Late Signal Test Waveforms …………………................................. 56

Fig. 4.7.4 Response Time Test ......................................................................... 57

Fig. 4.7.5 Faulty Checker SD6 on Chip 7 ......................................................... 58

Fig. 4.7.6 Disconnected Wire on Chip 7 ........................................................... 58

Fig. 5.1.1 Full Custom Design Flow …………………………............................. 60

Fig. 5.2.1 Standard Cell Dimensions ...................................................................... 62

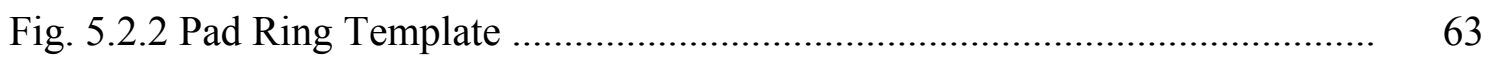

Fig. O.2.1 MOSIS Design Submission Flow ………….......................................... 101 


\section{CHAPTER 1}

\section{Introduction}

The advancement of electronic design methods and fabrication technology continues to drive the miniaturization of electronic devices, which in turn increases product reliability requirements. Studies have shown how transistor scaling impacts longterm product reliability [1]. Prior work has quantified scaling effects on lifetime reliability [2].

Common failures in chip operation are due to manufacturing defects, environmental influences, and structural deformations that occur over a long operating period. These defects manifest themselves in phenomena like electromigration $[3,4]$ and hot electron/hole injection $[5,6]$. These imperfections lead to reliability failures during operation [7].

Electromigration is the transport of mass in metals when they are stressed at high current densities [3]. The gradual movement of metal atoms creates voids in the conductor. These voids alter the electrical characteristics of the conductor, leading to signal delay and glitches that cause reliability failures.

Several methods for detecting reliability failures through on-line testing/selfchecking methods have been introduced [8,9]. One such method, called stability checking, was proposed by Franco and McCluskey to detect unstable and spurious output signals at sample times in sequential circuits [10]. 
Stability checkers concurrently monitor circuit outputs for errors during normal operation based on the system clock. These checkers are fundamentally Master-Slave flip-flops with a dynamic node that serves as the error detection mechanism. Like most CMOS logic circuits, the design of stability checkers for a specific application involves configuring device dimensions to fulfill the system's timing/frequency specifications.

The goals of this thesis are to construct and test Stability Checker designs, and to create a MOSIS [11] design template for future class projects in the EE 431 CAD of VLSI Devices course at Cal Poly. The stability checker designs are accompanied by configurable delay chains for on-chip test. The design project circuit form an expandable template library for potential student projects.

Chip design was done using free Computer Aided Design (CAD) tools, Electric VLSI Design Tool by Static Free Software [12] and LTSPICE by Linear Technology [13]. Consequently, chip fabrication and assembly are facilitated by the MOSIS service as part of the MOSIS Educational Program (MEP), under the Research Program.

The remainder of this thesis is organized as follows. Chapter two of this thesis presents a background on stability checking and related work, configurable delay techniques and fabrication service providers. Chapter three describes the entire design process from circuit design through layout verification and tapeout. Chapter four presents post-silicon inspection and verification results while chapter five describes the MEP for Cal Poly chip design projects. Finally, chapter six summarizes project findings and presents recommendations for future work. 


\section{CHAPTER 2}

\section{Background}

This chapter introduces stability checkers and configurable delay techniques, as well as application specific integrated circuit (ASIC) fabrication services. Section 2.1 defines stability checking concepts while section 2.2 examines related work on error detection. An on-chip error pattern generator facilitates test vector generation. Consequently, section 2.3 presents a survey variable delay techniques considered in this project. Section 2.4 identifies several ASIC fabrication service providers.

\subsection{Stability Checking}

On-line stability checking ensures data integrity in critical applications [10]. This technique was derived from a conventional delay testing method for faults caused by physical failure mechanisms [18].

Failures, such as electromigration, increase propagation delays in a circuit without causing catastrophic failures. Most CMOS reliability failure mechanisms are not instantaneous but instead degrade over time. Delay detection operating concurrently (online) with the system can identify these errors. Delay testing principles described in published literature [18] are reproduced below for improved comprehension on stability checking. 


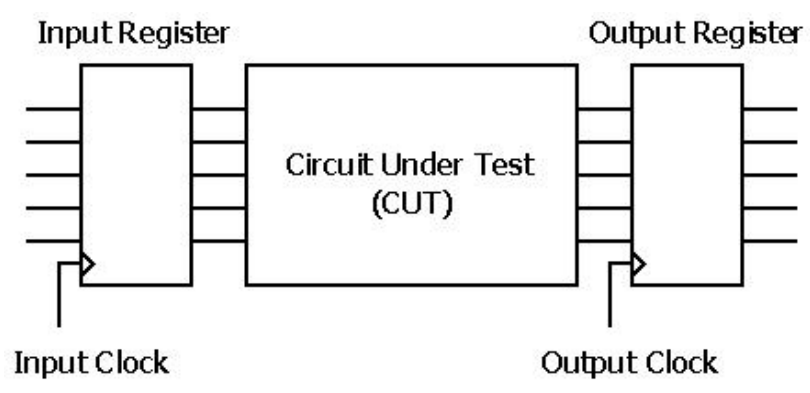

Fig. 2.1.1 Hardware Model [18]

\subsubsection{Delay Fault Testing}

Delay fault testing applies variable speed pseudorandom patterns to a circuit under test (CUT). Figure 2.1.1 illustrates the hardware model for conventional delay fault testing. The CUT is tested in a three step process. In the first step, an input pattern is clocked into the CUT to initialize it for some target test condition. In the second step, once the transients in the CUT have settled, a test vector is then clocked in excite logic level changes in the CUT outputs. The final step involves sampling the CUT output register.

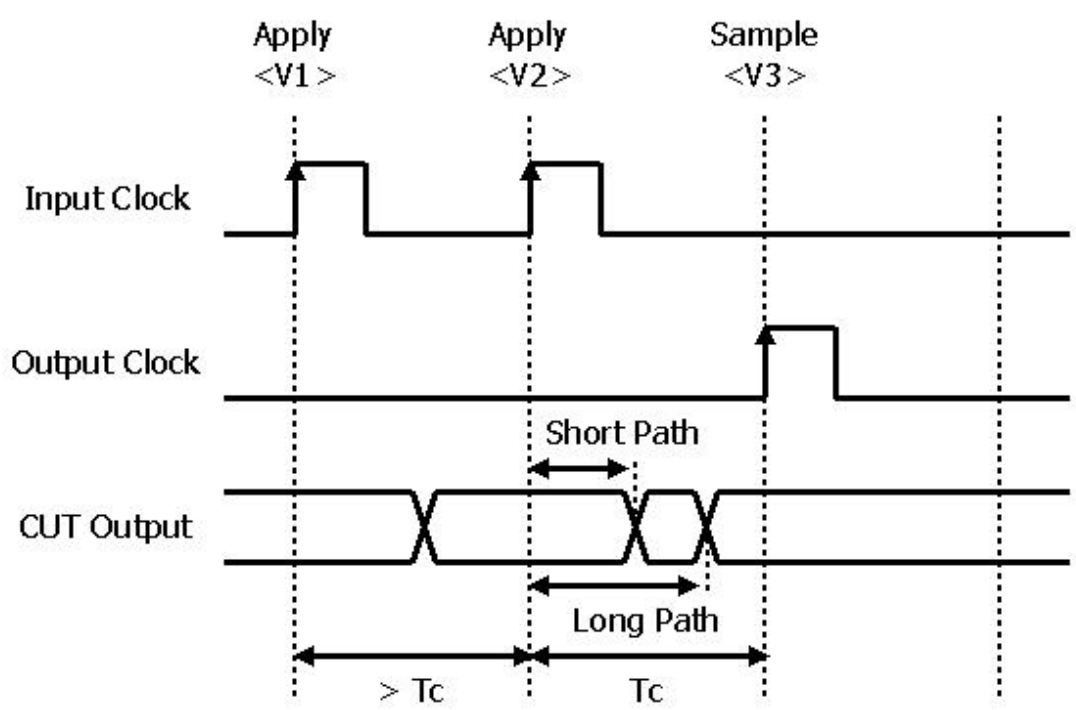

Fig. 2.1.2 Delay Fault Testing Waveforms [18] 
An off-line delay testing sequence is illustrated in Figure 2.1.2 where V1 is the initializing vector, V2, the test vector that launches transition propagation through the CUT, and V3, the output sample. V2 is applied after the cycle time, Tc, when transients in the CUT have settled.

\subsubsection{Limitations of delay fault testing}

Only a single sample output waveform sample is taken at V3. This poses the challenge of tuning sampling signal, V3, to accommodate timing requirements. Following that, the single sample limitation causes potential invalidation by dynamic hazards [18] (Fig. 2.1.3). The figure shows low to high signal transitions with switching. Each signal offset with different delays with respect to the sample time.

For a fault-free circuit, case I, the output waveform starts at logic 0 , pulses once before stabilizing at logic 1 before the sampling time. The waveforms in cases II and IV show delay faults, where the signal transitions were shifted in time, resulting in the wrong value being sampled, and which is detected as a delay fault. However, for a larger delay fault (case III) the momentary pulse is sampled as a fault-free condition, leaving the actual fault undetected. In this case, the test has been invalidated by the error. 


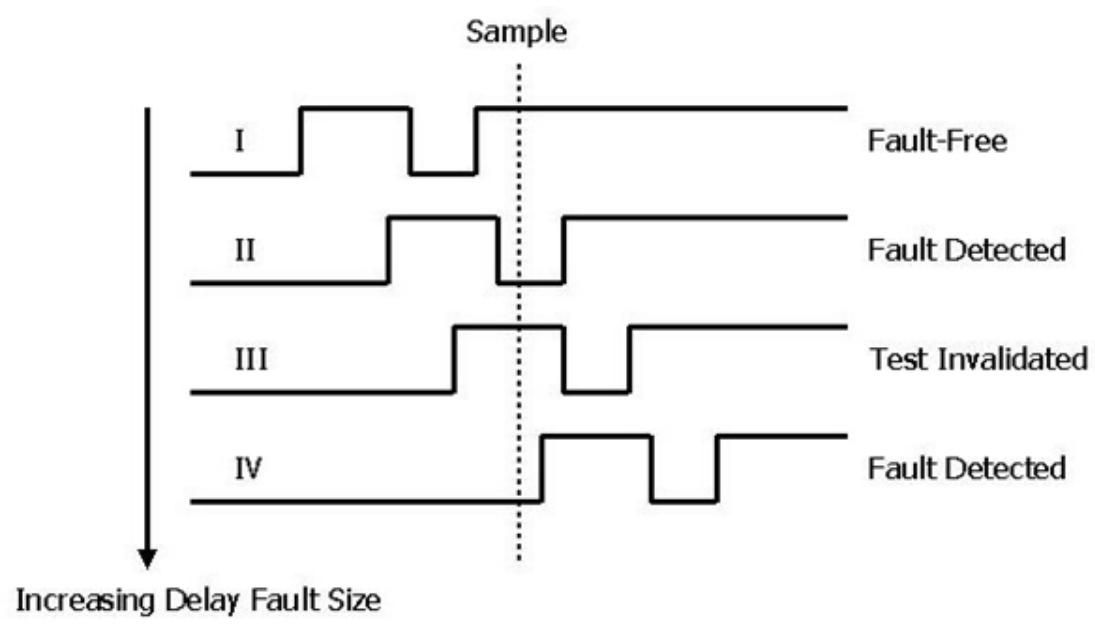

Fig. 2.1.3 Delay Test Invalidation by Dynamic Hazards [18]

\subsubsection{On-Line Stability Checking}

Stability checking [10] was introduced in an attempt to resolve the test invalidation problem. Stability checking observes the output waveform over a period of time after the sample. One of the key contributions of stability checking is to eliminate dependence on complex input test patterns. CUT output analysis allows testing in cases where inputs cannot be controlled, i.e.: on-line checking.

On-line stability checking assumes fully functional systems (no logic function errors). By extension, the outputs of a fault free circuit have stabilized by the time they are sampled. Hence, delay faults caused by wearout effects can be detected by monitoring the outputs for any changes in a time window after the sampling clock edge. The time interval during which the outputs are checked for stability is called the checking period, Tstab (Fig. 2.1.4). 
The challenge in implementing stability checking on-line is that applying $\langle\mathrm{V} 3\rangle$, which specifies the checking period, after $<\mathrm{V} 2>$ would affect the performance of the circuit. Therefore, $<\mathrm{V} 3>$ has to be shorter than the propagation delay of the shortest path in the circuit.

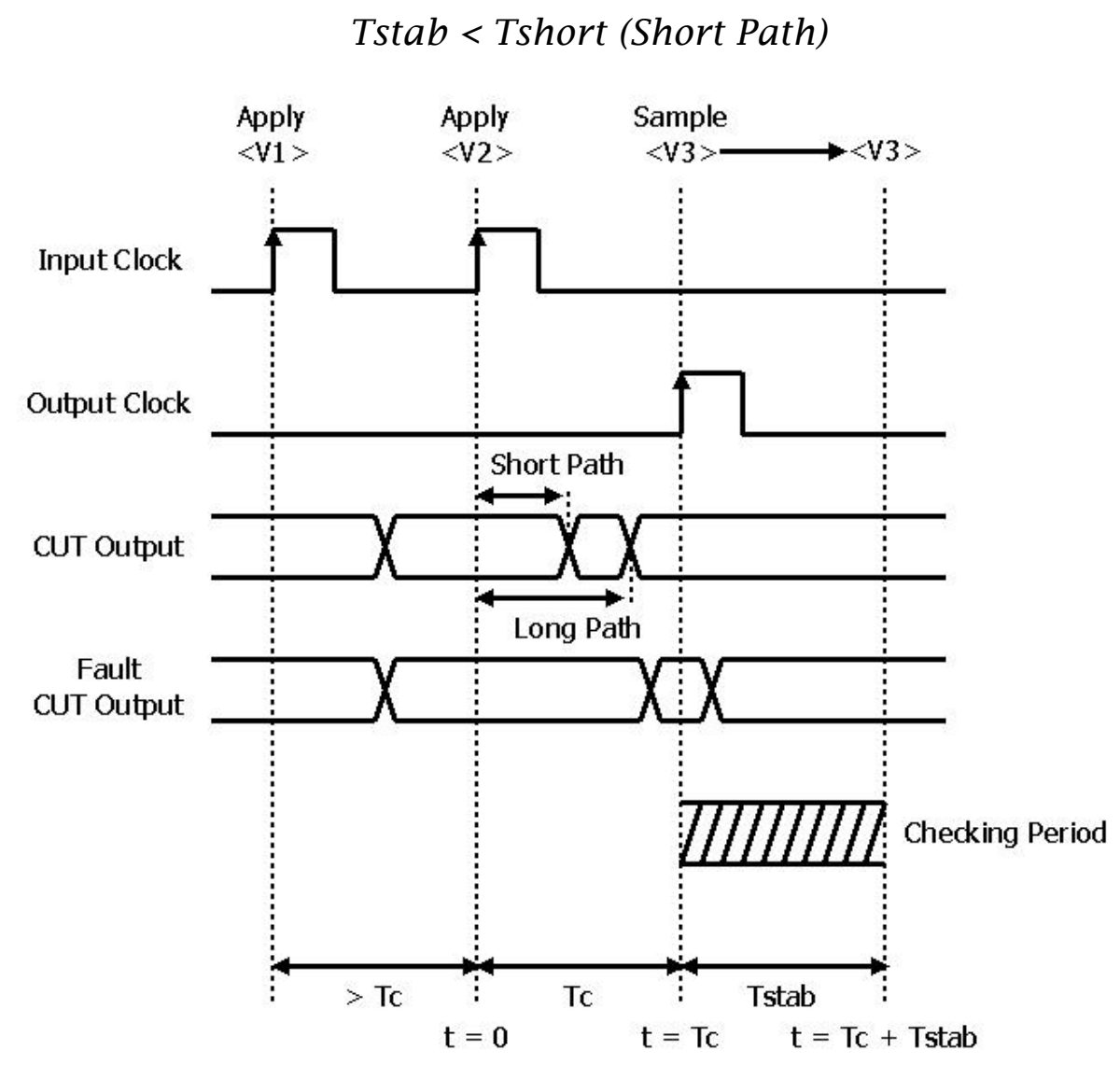

Fig. 2.1.4 Stability Checking Waveforms [10]

The direct approach in implementing a circuit for stability checking involves sharing logic with a system flip-flop using dynamic logic. In an edge triggered system, the checking period is duty cycle of the system clock of the flip-flop. Further details on stability checker design are described in Chapter 3. 


\subsection{Related Work on On-Line Testing}

On-line testing schemes are designed with four parameters in consideration [19]:

- Error coverage: The percentage of modeled errors that can be detected.

For example, stuck-at faults, bridging faults, open fault and delay faults.

- Error latency: The time period between error occurrence and detection. Safety-critical applications require lower error latency.

- Hardware redundancy: Logic overhead required for testing. Additional hardware required to perform on-line testing.

- Time redundancy: Time overhead required for error detection. For example, recomputing with shifted operands, swapped operands or duplication and comparison.

Various other on-line testing techniques have been introduced which fulfill the four parameters to different extents. Common techniques involve coding schemes that introduce additional hardware complexity. Examples of such methods are parity checking [20] [21] and self-checking systems [22]. Techniques that are invulnerable to hazard invalidations have also been discussed in detail in a series of studies [23] [24].

A method derived from stability checking was introduced by Yada et al. [25]. The modified stability checking technique is capable of detecting small delay faults during setup time (pre-sampling output waveform analysis). The design implements and XOR function during the checking period to detect unstable output signals. Although it requires additional transistors for the XOR logic, its modular design makes this technique highly scalable and simple to design. 


\subsection{Survey on Variable Delay Techniques}

Delay elements manipulate resistive-capacitive $(\mathrm{RC})$ loading on a target signal line. Adding, removing or reconfiguring MOS structures that alter parasitic effects influence signal speed. Variable delay elements exhibit differences in delay range and tuning precision. Consequently, parametric differences determine suitability for specific applications.

Variable delay elements are used to manipulate rising or falling transition times. They are used extensively in various applications, including digital delay locked loops, digitally controlled oscillators, deskewing clock distribution networks, as well as silicon debugging and path delay characterization. For this thesis, variable delays are used to generate test patterns for the stability checker circuits. This section reviews three common variable techniques:

- Current starvation technique, implemented by the digitally controlled delay element (DCDE) [26] [27]

- Shunt capacitor technique, implemented by the digital deskewing circuit for clock distribution networks [28]

- Variable resistors technique, implemented by a digitally adjustable resistor inverter [29]

\subsubsection{Current Starvation Technique}

The current starvation technique modulates currents driving the pull up and/or pull down loads in an inverter [26]. Fig. 2.3.1 shows a basic current starved delay 
element. It consists of a pair of inverters, M4-M5 and M7-M8 that act as a buffer. The input inverter M4-M5 is the current starved element.

The charging and discharging currents of the input inverter, M4-M5, are determined by the gate voltages of M3 and M6 respectively. The gate voltages of M3 and M6 limit the current drawn by the input inverter as it charges and discharges based on the input signal at In.

M3 and M6 are in turn are controlled by current mirror, M1 and M2. Further details on the architecture and operation of this technique are further described in literature [26].

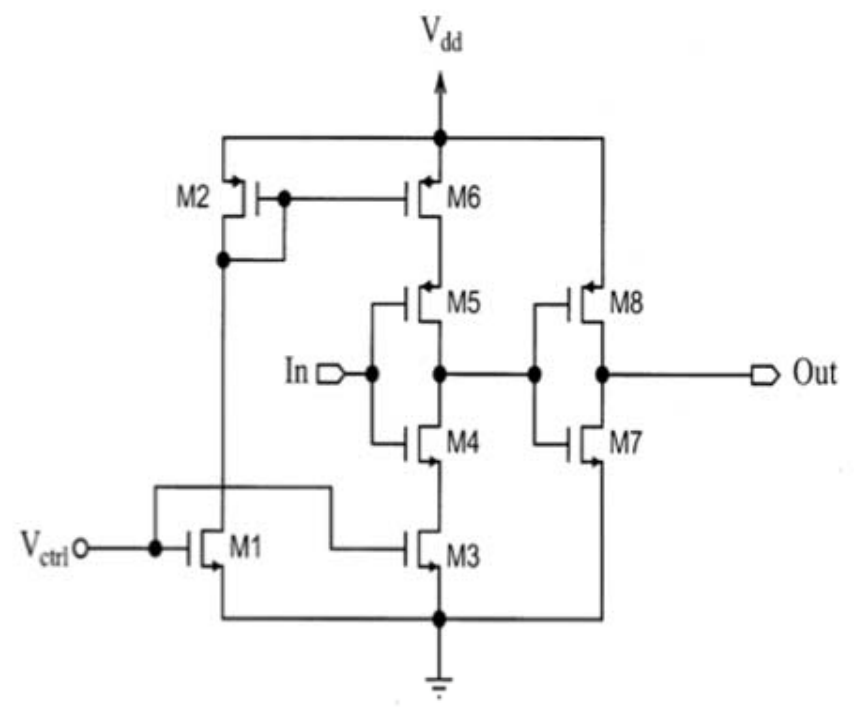

Fig. 2.3.1 Current Starved Delay Element [26]

The schematic for the DCDE with controlling transistors are shown in Fig. 2.3.2. Current starved transistors M8 and M11 determine the transition time of the inverter M9, M10. M8 and M11 are in influenced by current mirror, M5, M6 and M7. 
Transistor array M1 to M4 determine the magnitude of current, I, and provide a discrete control over the system via a digital vector. The $\mathrm{W} / \mathrm{L}$ ratios of these control transistors are sized in relative binary weights to obtain an incremental, monotonic delay.

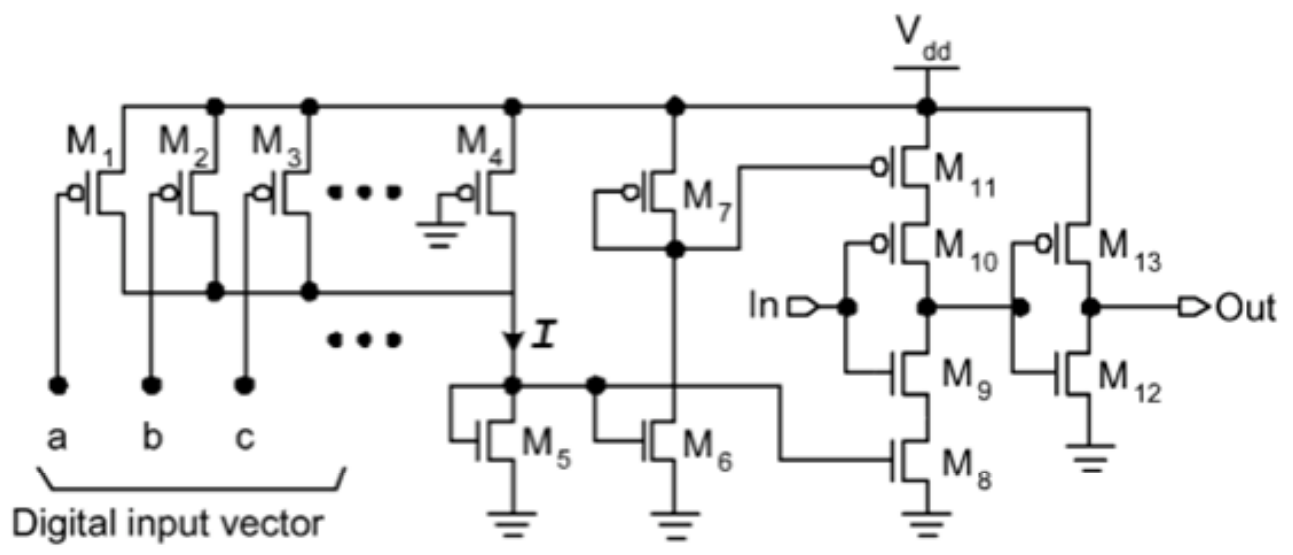

Fig. 2.3.2 DCDE Architecture [26]

\subsubsection{Shunt Capacitor Technique}

The shunt capacitor technique exploits transistor gate oxides capacitance to form capacitor cells. This method was developed at Intel [28] for clock deskewing. The capacitor cell shown in Fig. 2.4.3 uses a single transmission gate to attach or detach the capacitor pair to a delay line.

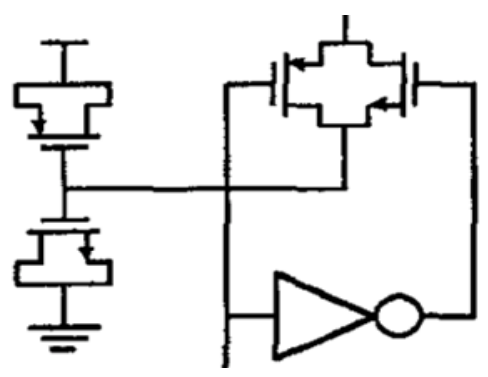

Fig. 2.3.3 Shunt Capacitor Cell [28] 
Capacitor cells are connected in a parallel chain to the delay line via the 'in/out' port. These capacitive loads are added or subtracted from the delay chain by a digital vector controlling cell enable lines. Fig. 2.3.4 shows clock deskewing using shunt capacitor cells.

As shown in Fig. 2.3.4, eight delay cells are connected to each both true and inverting phases of the delay line. The delay shift register stores the delay line state and is configured based on clock skew detected by a phase checker.

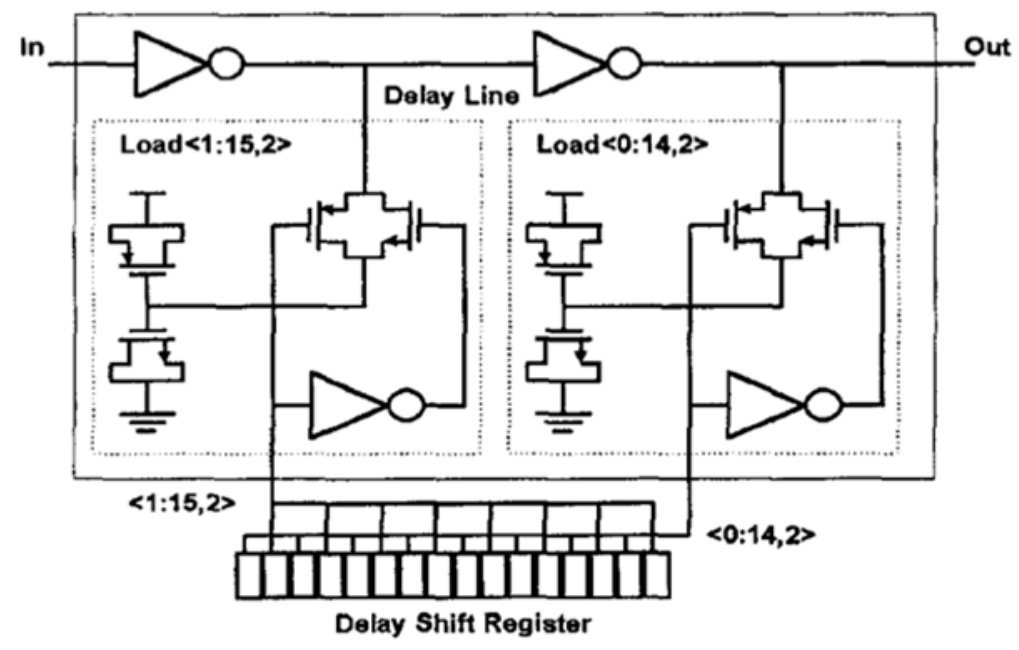

Fig. 2.3.4 Digital Deskewing Circuit [28]

The phase checker accepts clock lines from the system's time-critical sections and generates control signals based on detected skew to configure the variable delay shift register.

\subsubsection{Variable Resistor Technique}

This technique [28] is similar to the current starved transistor method, differing only in delay element fragmentation. Similar to using binary weight sized transistors to 
control a current source, this technique uses an array of NMOS or PMOS transistors connected directly to inverter source nodes in a two dimensional mesh. A transistor array at the inverter pull down stage provides multiple discharge paths, thus creating variable rising transition times. Fig. 2.3.5 shows the general topology for this technique.

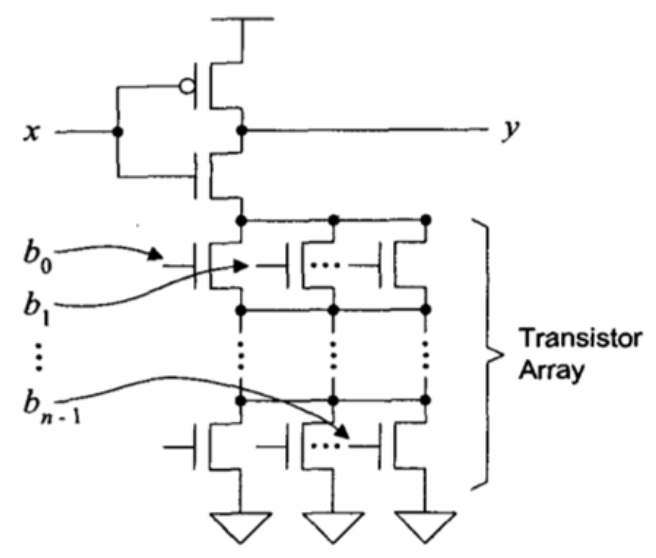

Fig. 2.3.5 Variable Resistor Configurable Delay Topology [28]

All control bits of any particular row cannot be simultaneously zero to maintain at least one path to ground, ensuring that the output can switch. For an $\mathrm{N}$ element array, there are theoretically $2^{\mathrm{N}}$ delay modes. However, choosing and determining the control vectors presents a coding problem in addition to sizing the transistors.

\subsubsection{Comparison of variable delay techniques}

The main objective of this comparison is to identify the technique with maximally uniform delay resolution to provide delayed signals to the stability checkers. The designs for each delay technique were implemented in SPICE based on a common set of parameters:

- circuits were designed for configurable rise transitions only 
- circuits were designed with 16 delay stages

- SPICE simulations with $1 \mathrm{~V}$ supply voltage at 40 Celsius

- transistor dimensions ranges (DCDE and Variable Resistor techniques):

$50 \mathrm{~nm}<\mathrm{L}<500 \mathrm{~nm}, 50 \mathrm{~nm}<\mathrm{Wn}<500 \mathrm{~nm}, 100 \mathrm{~nm}<\mathrm{Wp}<1000 \mathrm{~nm}$

From Fig. 2.3.6, it is clear that the techniques using binary weighted transistor sizing, DCDE and variable resistor, have non-uniform delay increments. It is also interesting to note the large delay contribution in the current starved element - control vectors 1 and 2 indicate modes where the current starved element is initially switched into the delay line. However, delay increments decay exponentially as the binary weighted elements are switched in.

In contrast, the shunt capacitor method provides more uniform delay resolution, i.e.: linear Trise response. Another advantage in the shunt capacitor technique is its modular architecture that simplifies design process.

The configurable delay technique used for testing stability checkers should have uniform delay increments and a simple control interface. A combination of the modular approach of the shunt capacitor method and current starved elements is adopted for the design of the configurable delay circuit. The variable resistor method was not suitable since the transistor array sizing and control vector coding was beyond the scope of this design. Further research on similar previously established techniques eventually led to another configurable delay chain topology that incorporated the current starvation approach with a modular cell-based design [39] which is described in section 3.3. 


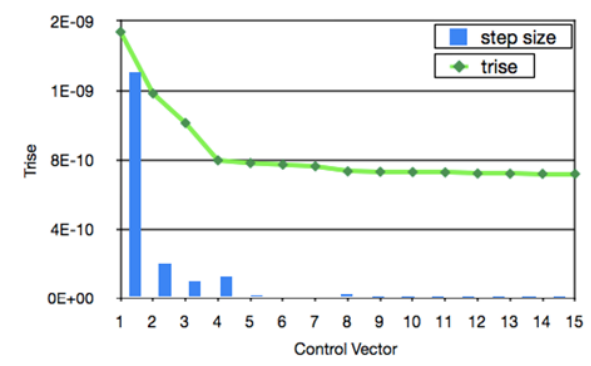

(a)

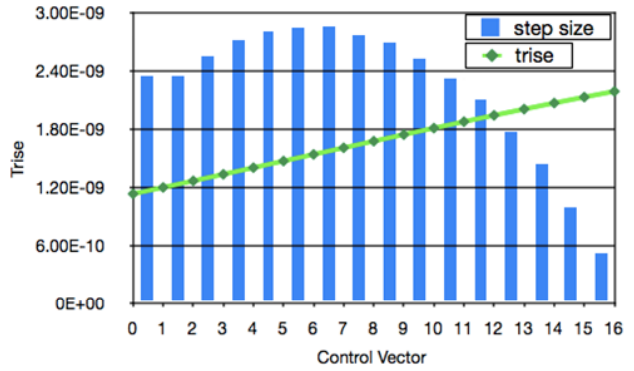

(b)

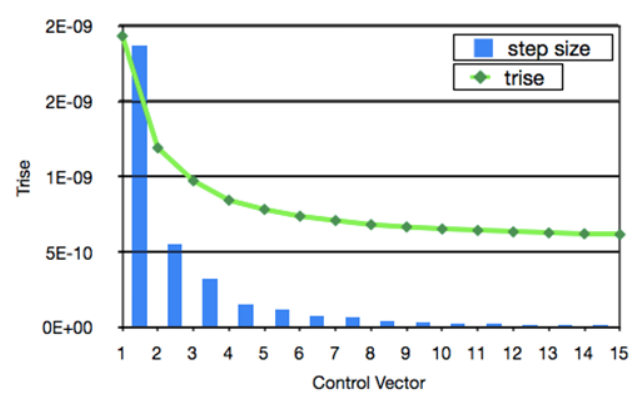

(c)

Fig. 2.3.6 Variable Delay SPICE Simulations (a) DCDE (b) Shunt Capacitor Technique (c) Variable Resistor Technique

\subsection{ASIC Fabrication Services}

MOSIS [11] provides ASIC fabrication services for low cost prototyping and low volume production. Other similar services include the EUROPRACTICE IC service [14] and Circuits Multi-Projets ${ }^{\circledR}$ Multi-Project Circuits ${ }^{\circledR}$, CMP [15]. These fabrication service providers are intermediaries between IC designers and partnering foundries (vendors), providing mask generation, wafer fabrication and device packaging services. In addition, designers are also provided process technology specific design kits (SPICE models, layout rules, etc.) by the associated vendor through the fabrication service provider.

MOSIS caters to academic projects at a reduced or subsidized cost. Commercial clients have access to all processes listed in Table 2.4.1 while academic projects are limited to older processes offered by IBM and ON Semiconductor. 


\begin{tabular}{|l|c|c|c|c|c|c|c|c|c|c|c|}
\hline \multirow{2}{*}{ Vendor } & \multicolumn{9}{|c|}{ Feature Size (nm) } \\
\cline { 2 - 14 } & 700 & 500 & 350 & 250 & 180 & 130 & 90 & 65 & 45 & 40 & 32 \\
\hline IBM & & $\bullet$ & $\bullet$ & $\bullet$ & $\bullet$ & $\bullet$ & $\bullet$ & $\bullet$ & $\bullet$ & $\bullet$ & $\bullet$ \\
\hline TSMC & & $\bullet$ & $\bullet$ & $\bullet$ & $\bullet$ & $\bullet$ & $\bullet$ & $\bullet$ & & $\bullet$ & \\
\hline ON Semi & $\bullet$ & $\bullet$ & $\bullet$ & & & & & & & & \\
\hline austriamicrosys & & & $\bullet$ & & $\bullet$ & & & & & & \\
\hline Globalfoundries & & & & & $\bullet$ & $\bullet$ & & $\bullet$ & & & \\
\hline Tezzaron & & & & & & $\bullet$ & & & & & \\
\hline Peregrine & & $\bullet$ & & $\bullet$ & & & & & & & \\
\hline
\end{tabular}

Table 2.4.1: MOSIS Fabrication Processes

The MOSIS service has been utilized by IC design courses at Boise State University and Harvey Mudd College. Senior and graduate IC design courses [16] taught by Dr. R. Jacob Baker at Boise State University involved the use of both Electric and LTSPICE to generate layouts for fabrication with the MOSIS service. In 2007, the E158 Introduction to CMOS VLSI Design class taught by Dr. David Harris at Harvey Mudd fabricated a 32-bit MIPS processor using Electric [17]. 


\section{CHAPTER 3}

\section{Chip Design}

This chapter describes the design process from component design, schematics, layout and verification through to tapeout. Section 3.1 presents an overview of chip subcomponents and process technology while sections 3.2 to 3.4 describe component design. Section 3.5 presents the final integrated design that was sent to MOSIS for fabrication.

\subsection{System Specifications}

The main system specification is the pad ring layout provided by MOSIS, and by extension, available chip packages with suitable cavity sizes. For the given 40 pin pad ring, the recommended package is a ceramic DIP by Kyocera [37], costing \$23 per die.

The purpose of this prototype chip is to provide a test platform for stability checker characterization. Consequently, the system was designed for eight stability checkers of varying error transistor dimensions, coupled with configurable delay modules for both the clock and data signal lines (Fig. 3.1.1). The 16-stage configurable delay modules are each controlled by a 4-bit vector via a one-cold decoder.

In addition to that, the system also provides ports for internal and external test signals. Test signals can be routed directly into the stability checker array or into the onchip configurable delay chains. 


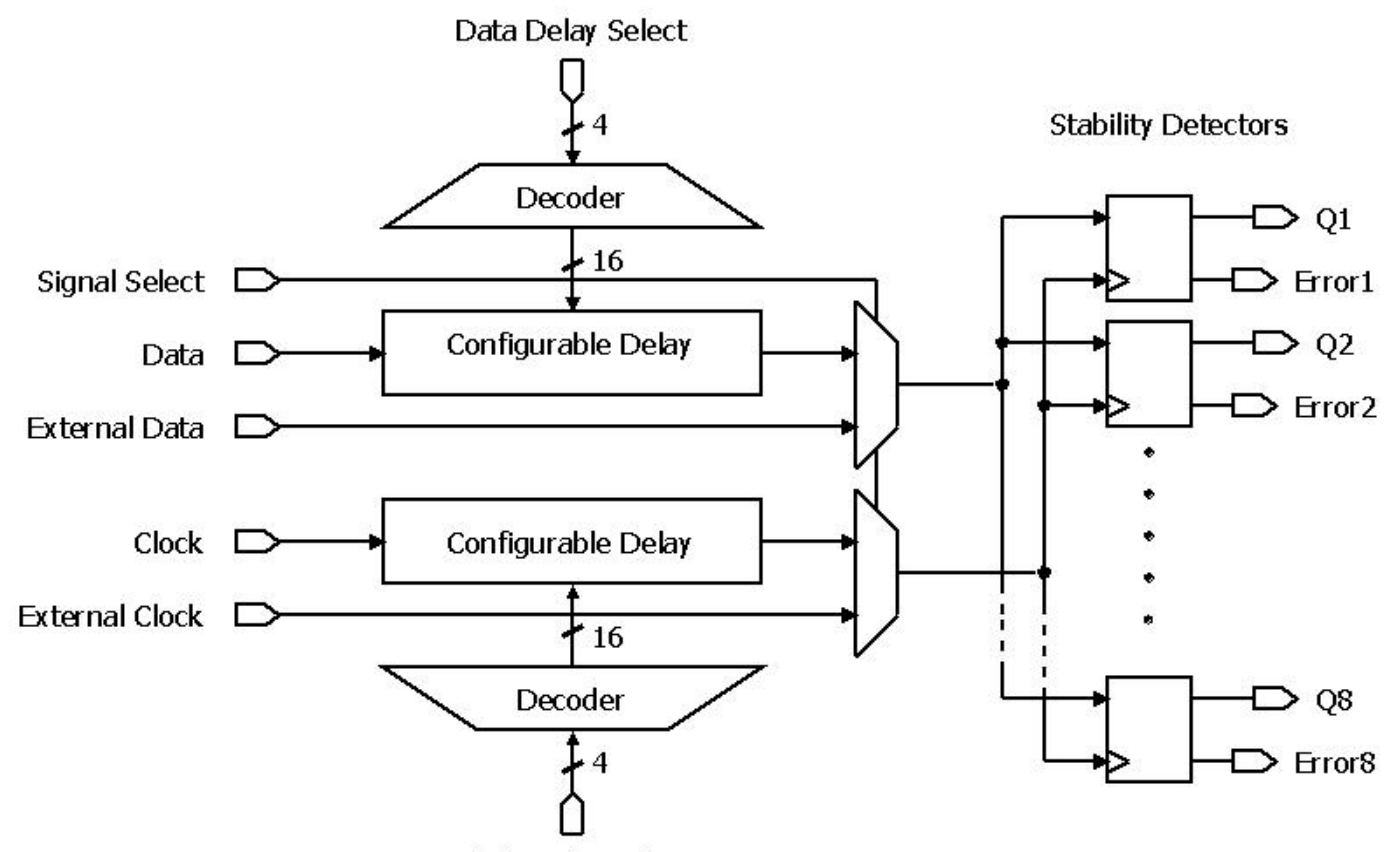

Clock Delay Select

Fig. 3.1.1 System Block Diagram

\subsubsection{Process Technology}

MOSIS provides Scalable CMOS (SCMOS) design rules that are compatible with the ON Semiconductor C5N process [38]. Electric is pre-configured with MOSIS SCMOS rules using a feature size, $\lambda$, of $0.3 \mu \mathrm{m}$. To verify design functionality, Electric writes the SPICE deck from its schematic/layout editor and invokes LTSPICE for simulations. Appendix C lists characterization data and CMOS SPICE models for the ON Semiconductor C5 process technology. This $0.5 \mu \mathrm{m}$ process operates at a $5 \mathrm{~V}$ supply voltage, $\mathrm{V}_{\mathrm{DD}}$. 
The $\mathrm{C} 5 \mathrm{~N}$ process provides 3 metal layers and a convention was defined for all layers:

- Metal 1 was used for cell supply rails ( $\mathrm{V}_{\mathrm{DD}}$ and ground) and to form logic/transmission gates. The connections are primarily aligned vertically.

- Metal 2 was used primarily for horizontal routing for transistors and logic/transmission gates within a cell. It is also used as an alternate routing for metal 3 lines that form inter-cell connections, to avoid metal 3 overlap.

- Metal 3 was used primarily for vertical routing for transistors and logic/transmission gates between cells. It is also used for clock distribution within stability checker cells.

As each CMOS cell was created using the layout instances provided by Electric,

the well and substrate were connected to power and ground implicitly [41]. This greatly simplifies the layout process as the designer is not required to separate substrate or well connections to the supply rails; but only has to ensure connections to the transistor source or drain terminals.

\subsection{Stability Checkers}

\subsubsection{Function}

Franco and McCluskey's stability checker designs were derived from transmission-gate Master-Slave flip-flops [36]. Fig. 3.2.1 shows an MS flip-flop schematic. It consists of two latches connected in series, with the input latch being the Master and the output latch being the Slave. Both latches are assigned complementary 
clock signals. The flip-flop passes the signal from input ' $d$ ' to output ' $q$ ' only during the rising clock transition. At all other times, the flip-flop retains its previous state.

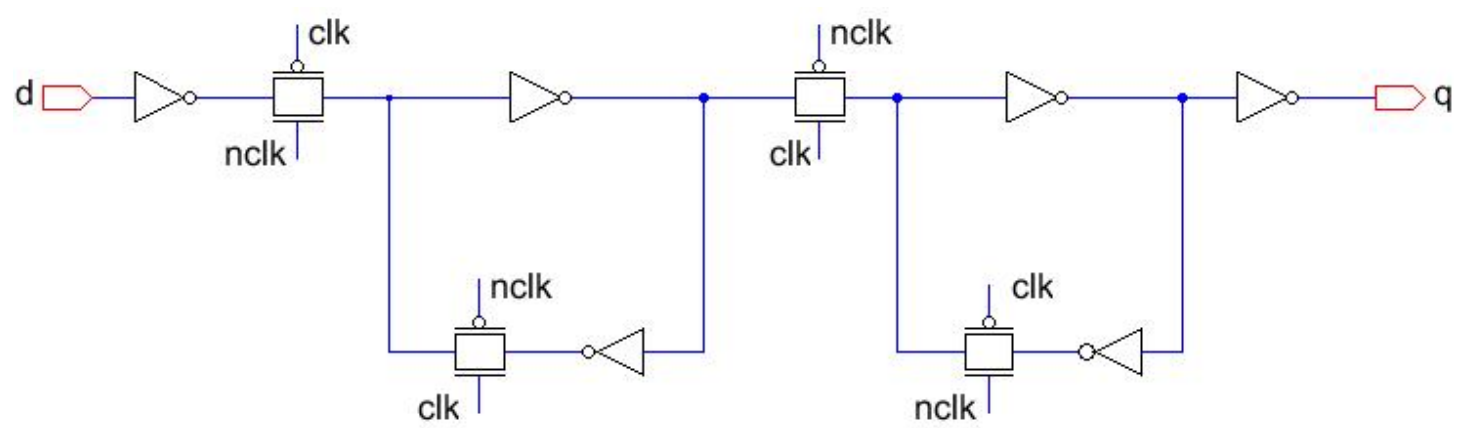

Fig. 3.2.1 Master-Slave Flip-flop Schematic

The stability checker design is based on this flip-flop. The critical observation from a fault-free system is that the input signal, 'd', should be stable after the sampling clock edge. Any changes in the input signal during the time period after the sampling edge should be identified as an error. Consequently, Franco and McCluskey redesigned the input latch to include a dynamic node that discharges if the input changes during the checking period.

All checkers function as regular flip-flops under normal conditions - no unstable or late signals. In the event of a late or unstable Data signal, the error transistor flags the error with a logic high output. The checker resets its error output at the end of the checking period.

\subsubsection{Schematic}

The stability checkers were designed for rising clock edge sampling. As mentioned previously in section 2.1 .3 , the checking period defines when the outputs of a flip-flop are checked for errors. The workaround to the problem of asserting a signal to 
start and end the checking period is to utilize the High half cycle of the system clock [10]. Thus, the rising edge of the sampling clock indicates the start of the checking period while its falling edge marks the end.

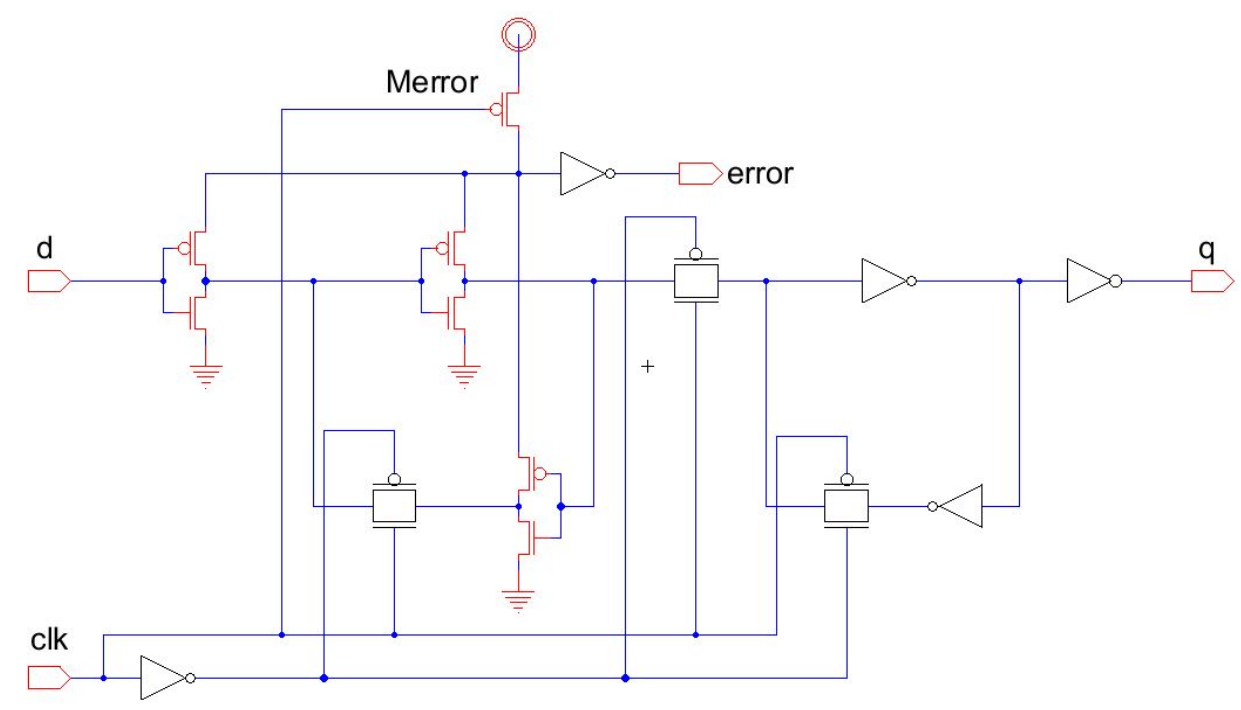

Fig. 3.2.2 Stability Checker Schematic

The drain of the error transistor, Merror, is shared with the inverter PMOS sources in the Master stage of the flip-flop to form a dynamic node (Fig. 3.2.2). When the clock (clk) is low before the rising sampling edge, Merror is $\mathrm{O}$ and the dynamic node is charged to approximately $V_{D D}-V_{t h, p}$, where $V_{\text {th, }}$ is the threshold voltage of a PMOS transistor.

At the rising sampling edge of the clock, the logic value latched in the Master portion of the flip-flop is transferred to the Slave output (q). After the rising edge, the Slave latches this value, ignoring any changes in the data line input (d) to the Master portion of the flip-flop. If the logic value at ' $\mathrm{d}$ ' is stable during the checking period, the dynamic node will retain its charge of about $\mathrm{V}_{\mathrm{DD}}-\mathrm{V}_{\mathrm{th}, \mathrm{p}}$, which in turn keeps the 'error' output at logic Low. 
During the checking period, if a stability fault or late signal event occurs at 'd', the switching activity (current discharge) of the inverters in the Master portion of the flipflop would cause a voltage drop at the dynamic node. This voltage drop causes a logic level change in the dynamic node and is indicated by a High at the 'error' output. The error signal resets to a Low at the Low half cycle of the clock.

\subsubsection{Layout}

The checkers are designed with minimum sized transistors based on the C5 process $-\mathrm{W} / \mathrm{L}$ ratio of $10 / 2(3.0 \mu \mathrm{m} / 0.6 \mu \mathrm{m})$. Each checker was drawn with discrete transistors that form the inverters and transmission gates (no diffusion sharing). Minimum length transistors were abutted to form continuous $\mathrm{P}$ and $\mathrm{N}$ wells. Fig. 3.2.3 shows the layout of a stability checker cell with a 10/2 error transistor located at the top right corner.

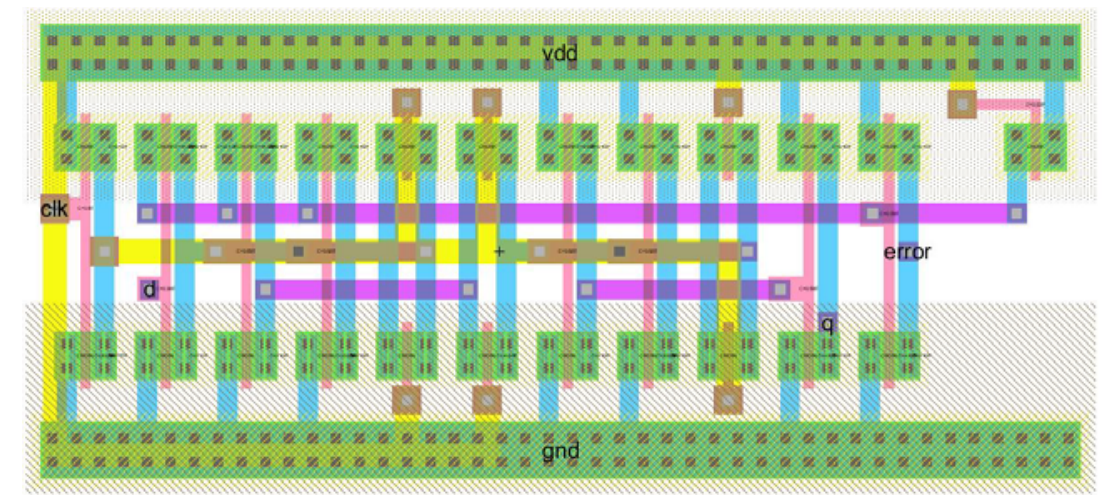

Fig. 3.2.3 Stability Checker Layout with 10/2 Error Transistor

Eight checker cell variants were designed based on error transistor dimensions listed in Table 3.2.1. To achieve transistor lengths, $L$, greater than $2 \lambda$, several transistors are chained end-to-end fashion (in series) with a common gate. As for transistor widths, 
$\mathrm{W}$, greater than $10 \lambda$, transistor folding is used - parallel diffusion-contact regions are drawn with common source and drain nodes. Fig. 3.2.4 shows eight stability checkers dimensions corresponding to Table 3.2.1 entries.

\begin{tabular}{|c|c|c|c|}
\hline Stability Checker & Width/Length, W/L $(\lambda / \lambda)$ & Width, $\mathrm{W}(\mu \mathrm{m})$ & Length, $\mathrm{L}(\mu \mathrm{m})$ \\
\hline SD0 & $10 / 2$ & 3 & 0.6 \\
\hline SD1 & $10 / 4$ & 3 & 1.2 \\
\hline SD2 & $10 / 8$ & 3 & 2.4 \\
\hline SD3 & $10 / 16$ & 3 & 4.8 \\
\hline SD4 & $20 / 16$ & 6 & 4.8 \\
\hline SD5 & $40 / 16$ & 12 & 4.8 \\
\hline SD6 & $40 / 8$ & 12 & 2.4 \\
\hline SD7 & $20 / 8$ & 6 & 2.4 \\
\hline
\end{tabular}

Table 3.2.1: Error Transistor Dimensions
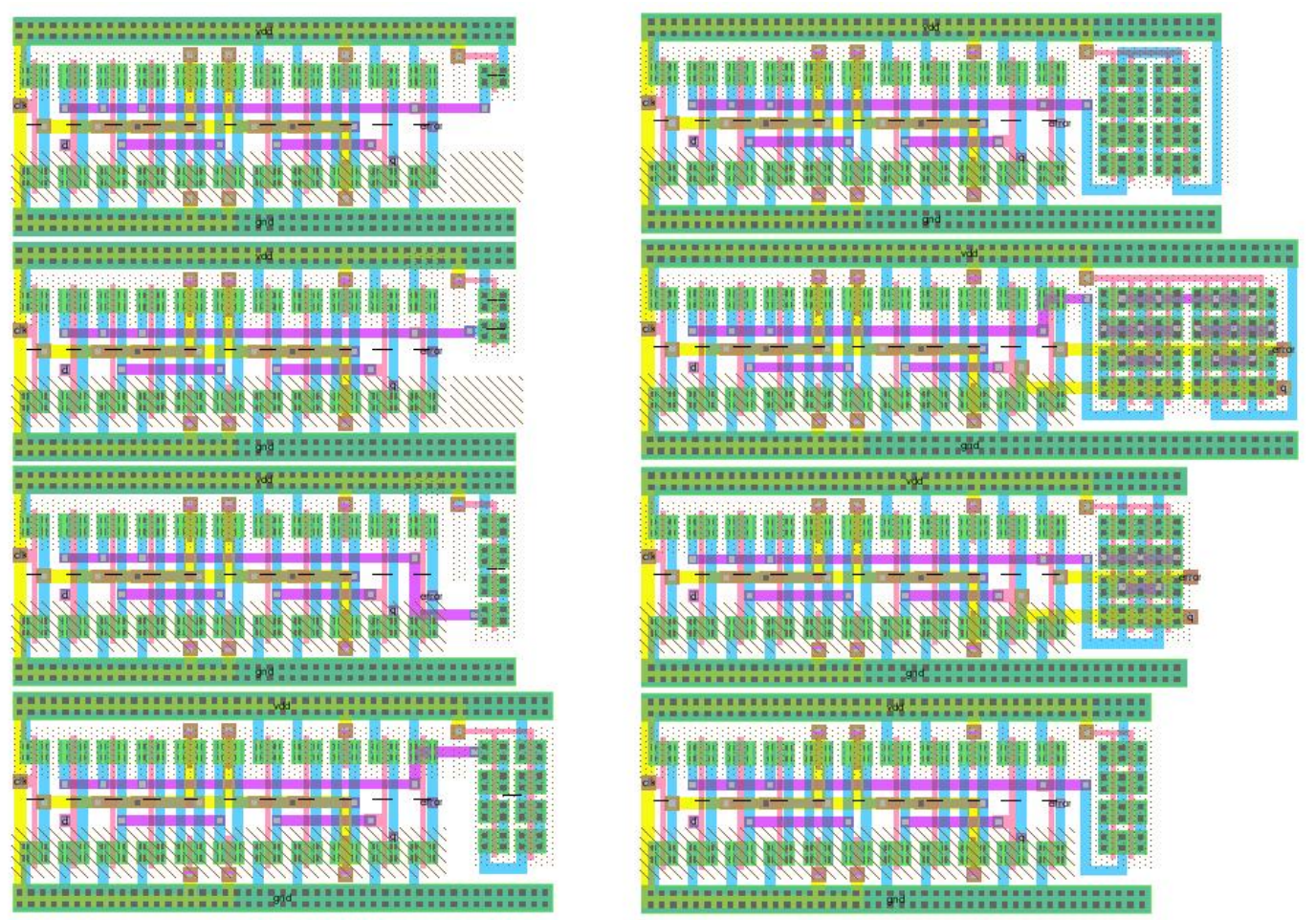

Fig. 3.2.4 Stability Checker Variants 


\subsubsection{Verification}

Each stability checker schematic and layout was verified for three functionality aspects. Fig. 3.2.5 shows the output waveforms of an operational stability checker design. The functional simulation verifications were conservative:

- No timing restrictions were imposed on checker operating frequency.

- Error patterns did not test for flip-flop setup and hold time requirements

The first requirement is that the stability checker must not impede regular flipflop operations. This is observed at the $3 \mu$ s mark, where the data input $V(d)$ has stabilized at the sampling clock edge $\mathrm{V}(\mathrm{clk})$ and output $\mathrm{V}(\mathrm{q})$ correctly displaying the input value. The error signal, V(error), remains Low, indicating that no error had occurred.

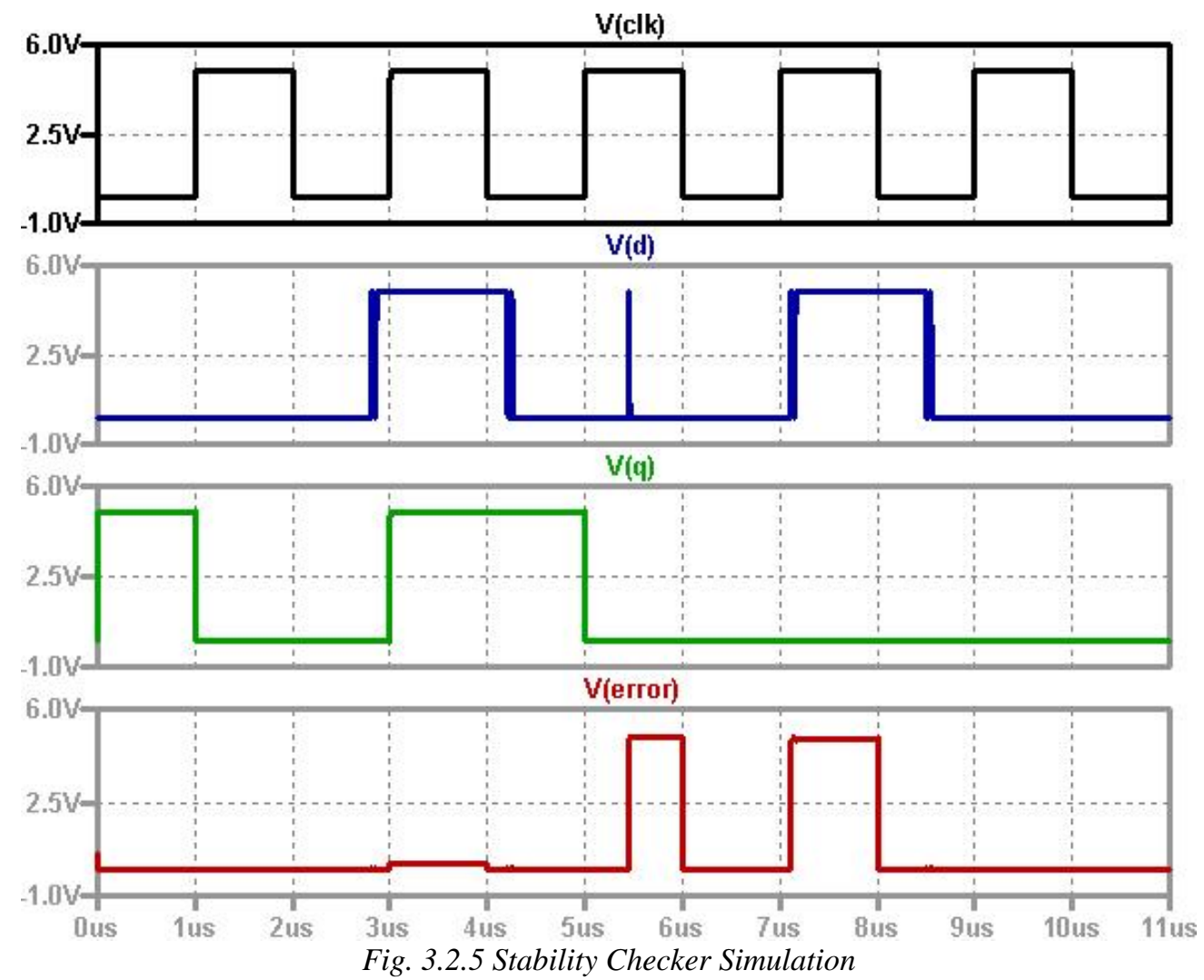


Secondly, checkers were tested for unstable signal detection. An unstable signal was modeled by a glitch in the $5 \mu$ s to $6 \mu$ s interval on V(d). V(error) correctly captured the glitch during the interval and reset at the end of the V(clk) High half cycle.

The final test ensures that the checker captures a late signal arrival event. A late signal was modeled by time shifting the input pulse $V(d)$ after the sampling $V(c l k)$ edge at $7 \mu$ s. The checker accurately captures the late signal on V(d) by setting V(error) to High and resetting at the end of the checking period.

\subsection{Configurable Delay Chain}

\subsubsection{Function}

As mentioned in section 2.3.4, the configurable delay technique used for this design was based on a combination of the modular approach of the shunt capacitor method with current starved elements. A recently patented configurable delay method based on a chain of stacked inverters [39] has many similarities to the principles discussed previously. This new method uses the current starvation technique to form delay cells, which are then chained together and coupled with switching logic to control the delay applied to a signal line.

The configurable delay chain module used in this design includes a single delayed signal line and 16 delay stages. Consequently, the delay stages are switched in on a 16-bit active-high control input. For every additional delay stage, the previous delay stages must remain connected. 


\subsubsection{Schematic}

The configurable delay module was constructed in 3 levels: a stacked inverter buffer, delay cell, and a top-level delay chain (Fig. 3.3.1). The stacked inverter consists of 2 PMOS in series and 3 NMOS in series. The W/L sizes for both PMOS and NMOS transistors were $5 / 2(1.5 \mu \mathrm{m} / 0.6 \mu \mathrm{m})$.

The delay cell in Fig. 3.3.1 (b) and Fig. 3.3.2 (a) consists of a stacked inverter buffer indicated as a buffer symbol in a rectangle. This cell incorporates the switching signal, 's', that routes either one of 2 inputs to the 'out' port:

- ' in' routes the delayed signal from the next stage.

- 'delay_in' routes the delayed signal from the previous stage.

Cells are connected in series as shown in Fig. 3.3.1 (c) and Fig. 3.3.2 (b). A delay cell is activated by asserting a Low at the ' $\mathrm{s}$ ' line. This specifies the activated cell as the last delay stage in the current mode. Setting 's' to High routes the delayed signal from the next stage to the current cell's input. 

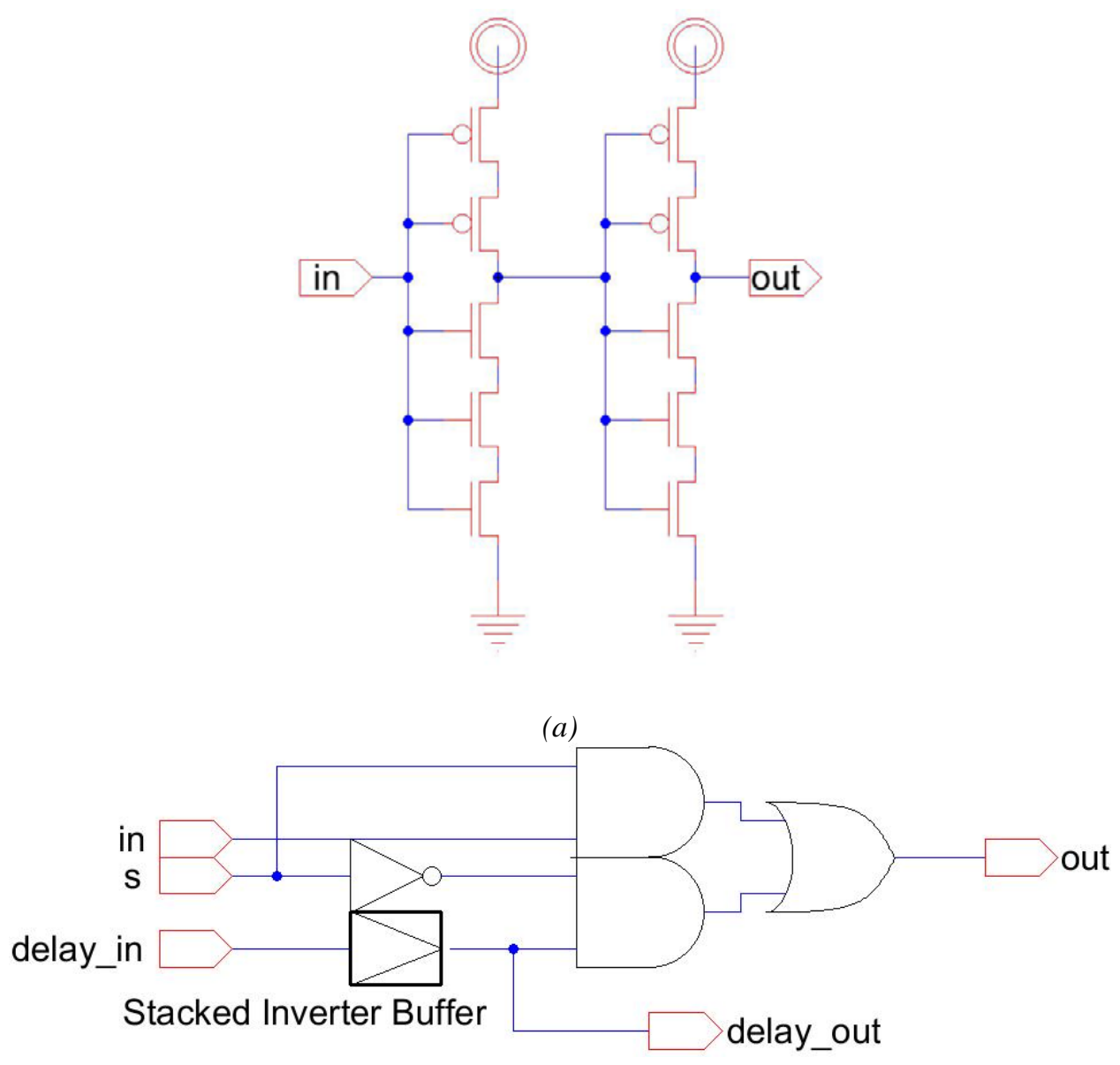

(b)

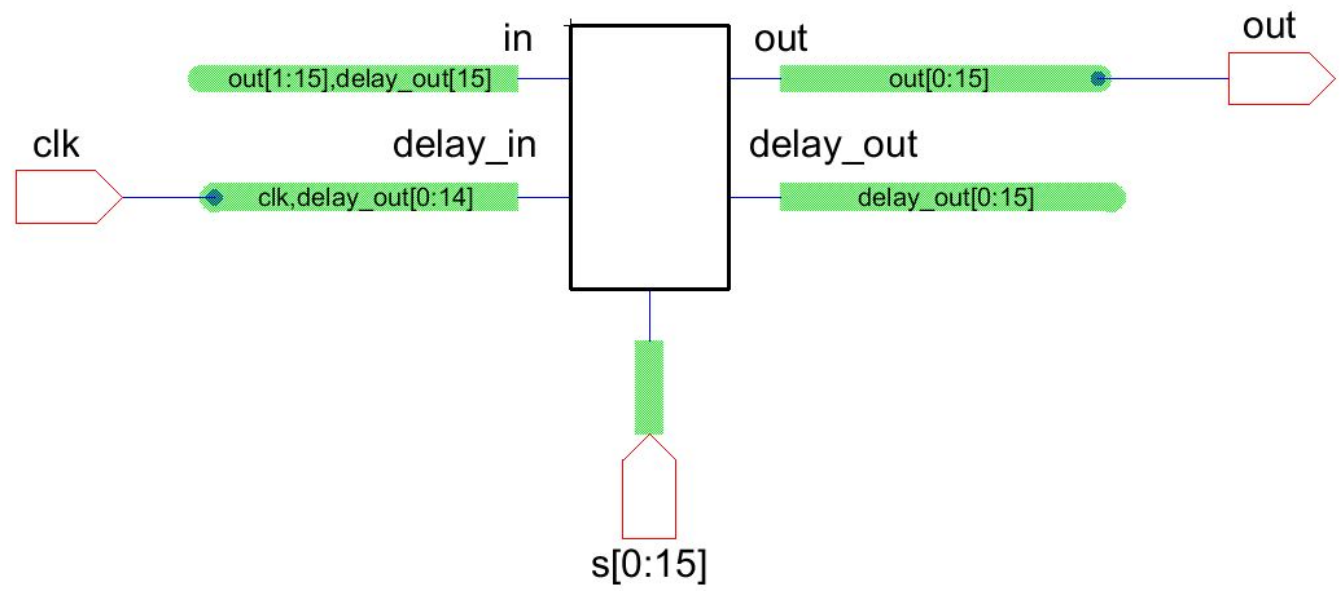

(c)

Fig. 3.3.1 Configurable Delay Schematic (a) Stacked Inverter Buffer (b) Delay Cell (c) Top Level Schematic 


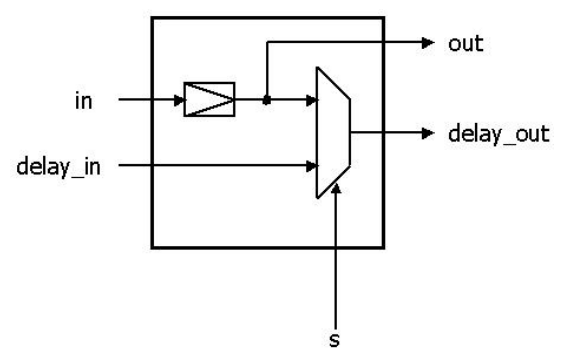

(a)

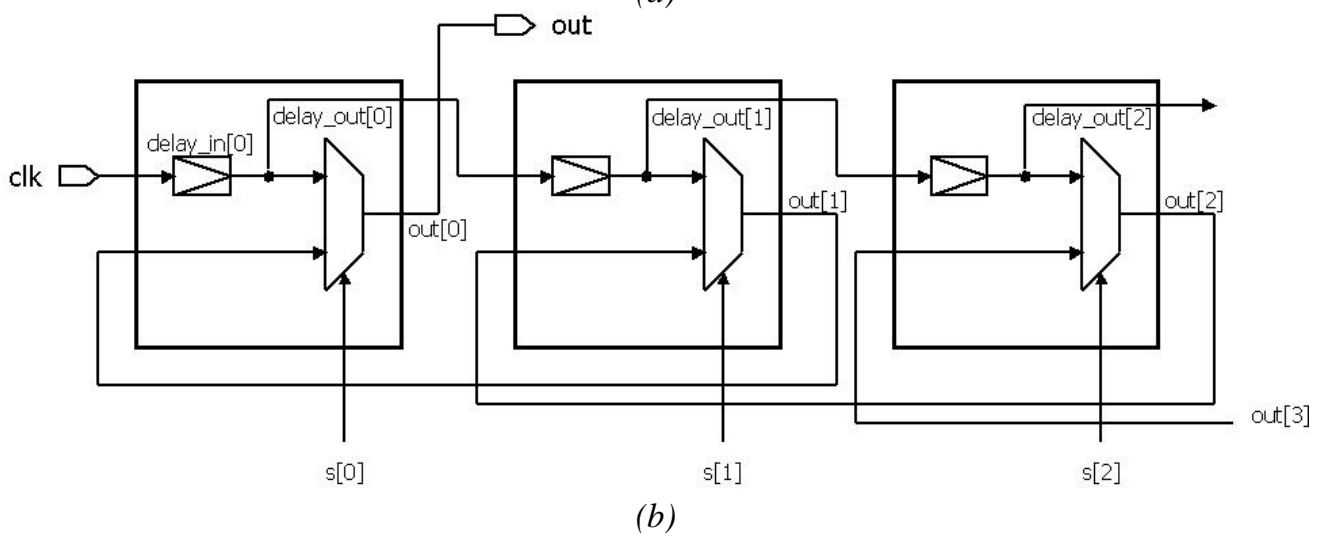

Fig. 3.3.2 Configurable Delay Block Diagram (a) Delay Cell

(b) Top Level of First 3 Delay Cells

\subsubsection{Layout}

The layout for this module was created hierarchically beginning from the stacked inverter buffer cell. Cell heights were set at $100 \lambda$ and transistors were aligned $8 \lambda$ from the supply rails. Layouts for the stacked inverter buffer, delay cell, and top level design are shown in Fig. 3.3.3. The stacked inverter layout was drawn with additional unused area to facilitate cell redesign for larger/smaller delay variants. The top level module consists of four staggered of four delay cells each. This staggered layout simplified intercell routing and greatly improved DRC checking time. 


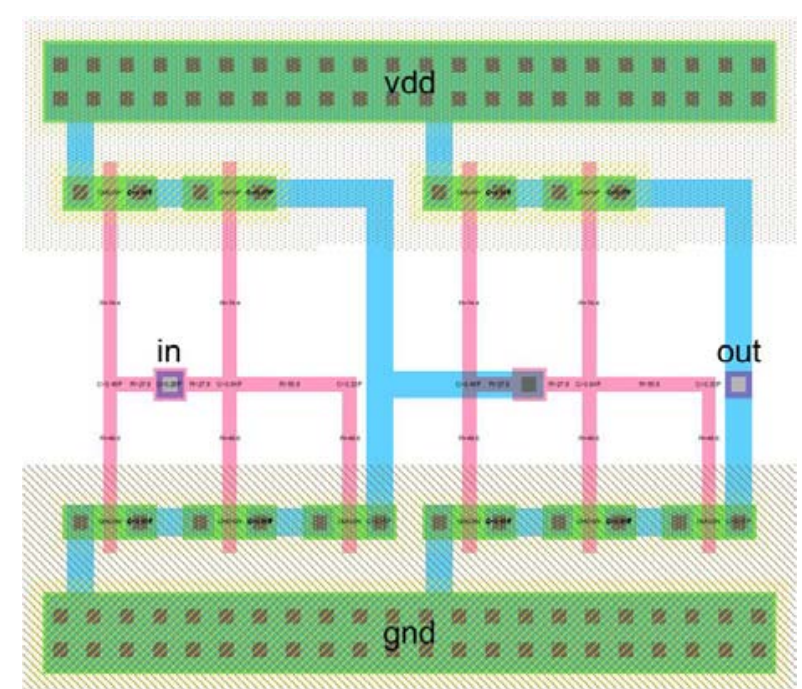

(a)

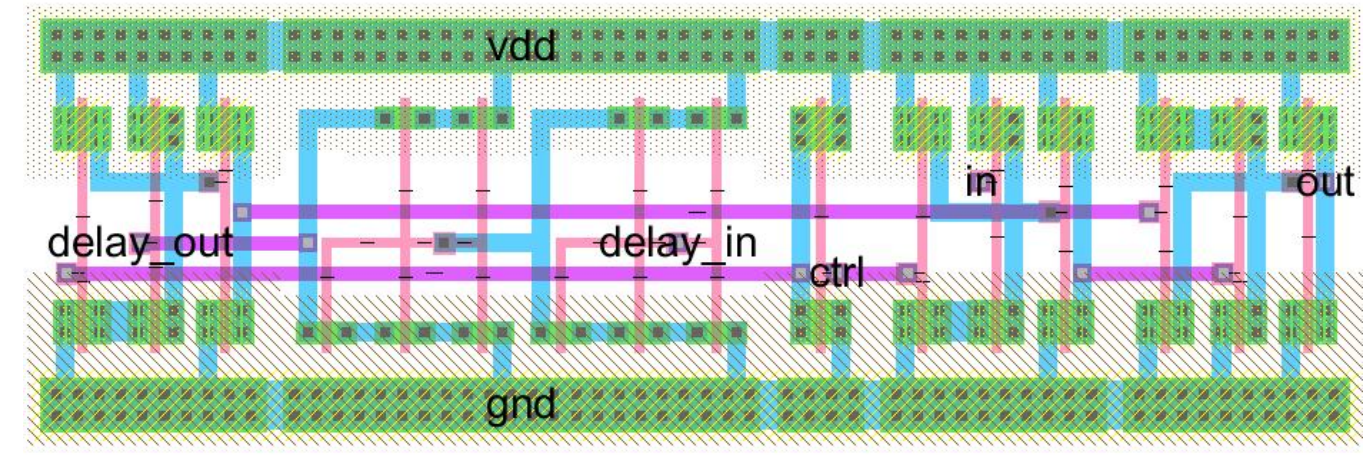

(b)

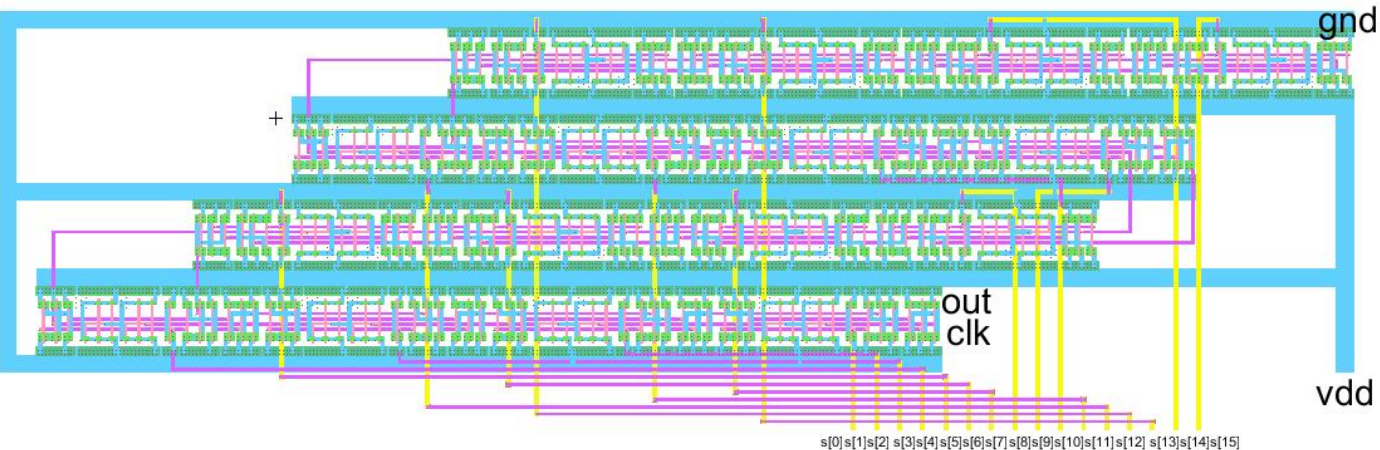

(c)

Fig. 3.3.3 Configurable Delay Chain Layout (a) Stacked Inverter Buffer (b) Delay Cell (c) Top Level Layout 


\subsubsection{Verification}

The simulated clock signal has a rise time of $0.1 \mathrm{~ns} ; 16$ iterations were completed for each delay stage. The delay waveforms for the first eight stages are shown in Fig. 3.3.4. Delay measurements were taken from the $50 \%$ crossing of the input rising signal to the $50 \%$ point of the corresponding delayed output. Fig. 3.3 .5 plots the simulated delays for all stages. The average delay increment is $0.771 \mathrm{~ns}$.
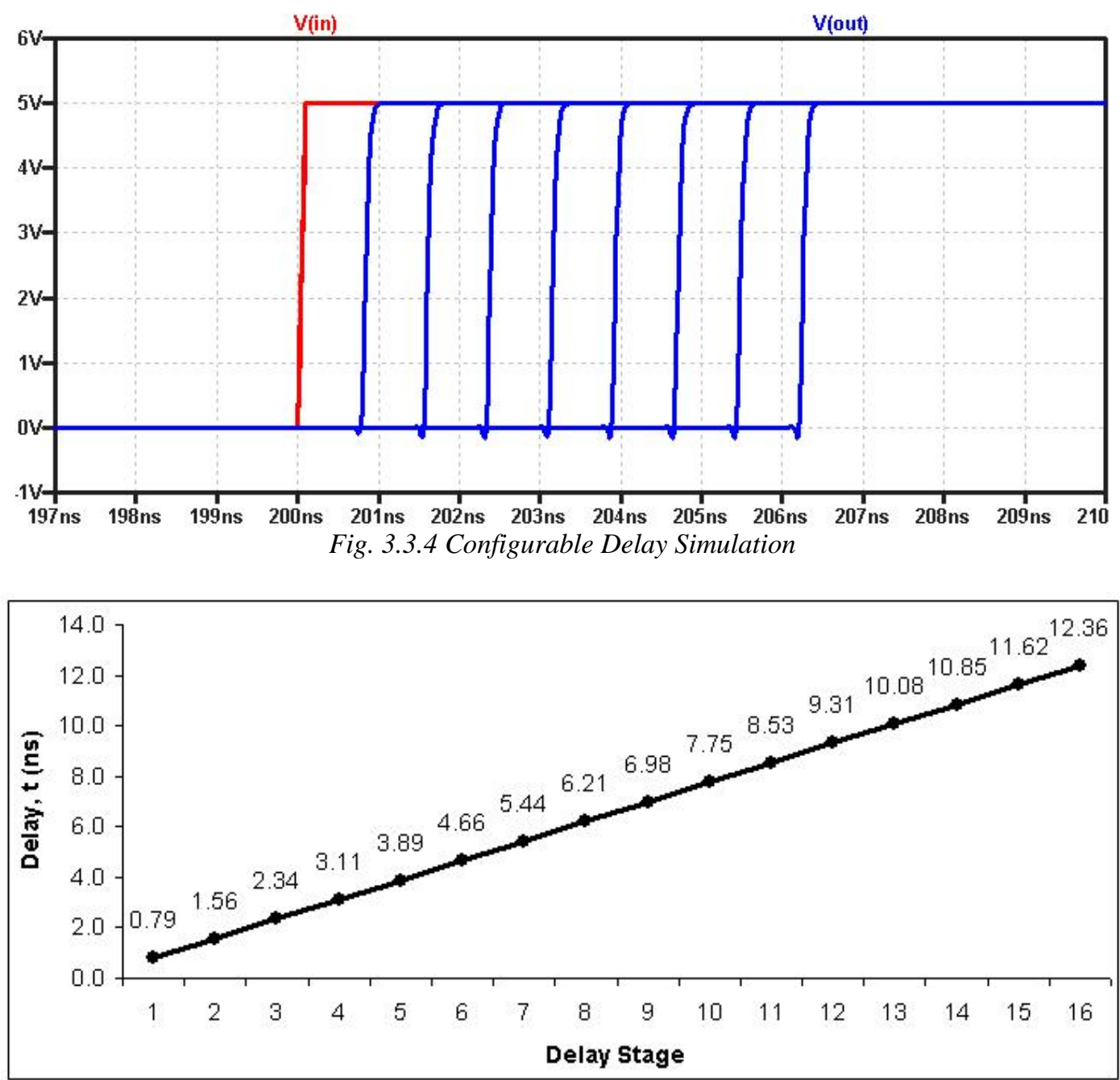

Fig. 3.3.5 Simulated Delay Measurements 


\subsection{One-Cold Decoder}

\subsubsection{Function}

This decoder was designed to provide a 4-bit interface to the 16-stage configurable delay chain. Each input state enables 1 of 16 configurable delay modes. The delay cell associated with the current mode has its control line set to Low while all other cells are set to High. The truth table of the one-cold decoder is shown in Table 3.4.1.

\begin{tabular}{|c|c|}
\hline Input, $\mathrm{A}[3: 0]$ & Output, X[15:0] \\
\hline 0000 & 1111111111111110 \\
\hline 0001 & 1111111111111101 \\
\hline 0010 & 1111111111111011 \\
\hline 0011 & 1111111111110111 \\
\hline 0100 & 1111111111101111 \\
\hline 0101 & 1111111111011111 \\
\hline 0110 & 1111111110111111 \\
\hline 0111 & 1111111101111111 \\
\hline 1000 & 1111111011111111 \\
\hline 1001 & 1111110111111111 \\
\hline 1010 & 1111101111111111 \\
\hline 1011 & 1111011111111111 \\
\hline 1100 & 1110111111111111 \\
\hline 1101 & 110111111111111 \\
\hline 1110 & 101111111111111 \\
\hline 1111 & 0111111111111111 \\
\hline Table 3.4.1: Decoder Truth Table
\end{tabular}

\subsubsection{Schematic}

The decoder architecture was implemented using 4-input OR gates based on the truth table.. As depicted in Fig. 3.4.2, the decoder generates complements for each input bit, a[n]. A buffer was inserted after every four connections to the OR gate array. 


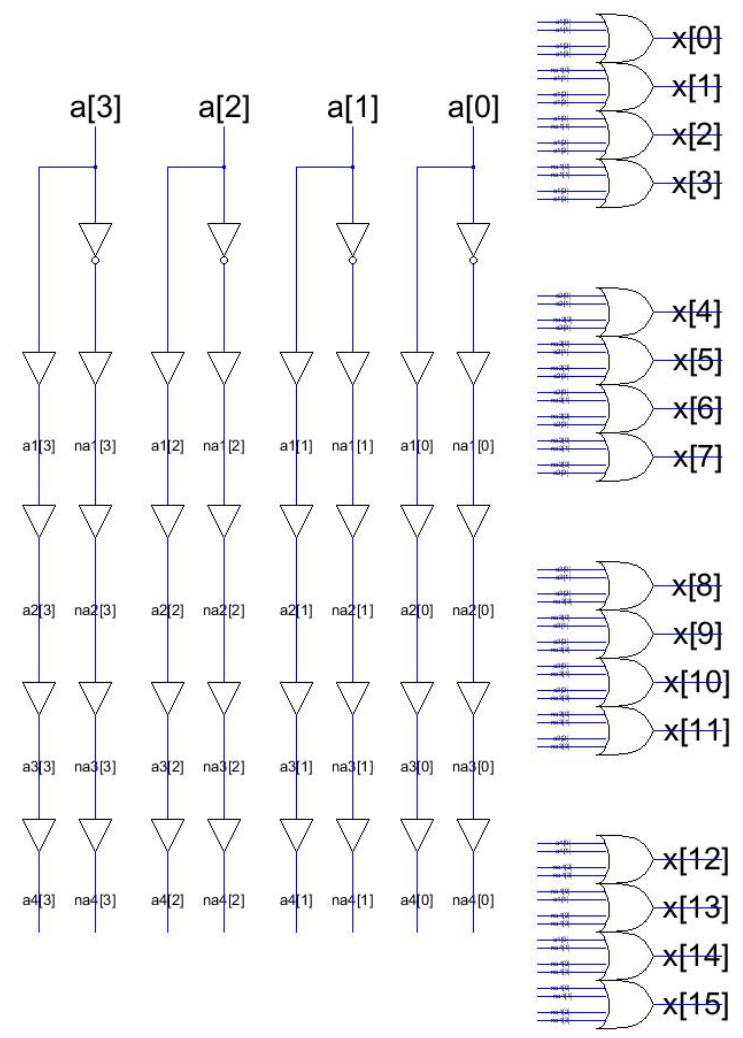

Fig. 3.4.2 Decoder Schematic

\subsubsection{Layout}

The decoder was laid out by abutting OR gate cells in a row and routing them to the corresponding bit lines. With a large available chip area and to facilitate manual routing, the layout was distributed over a broad region. Fig. 3.4.3 illustrates the top level decoder layout.

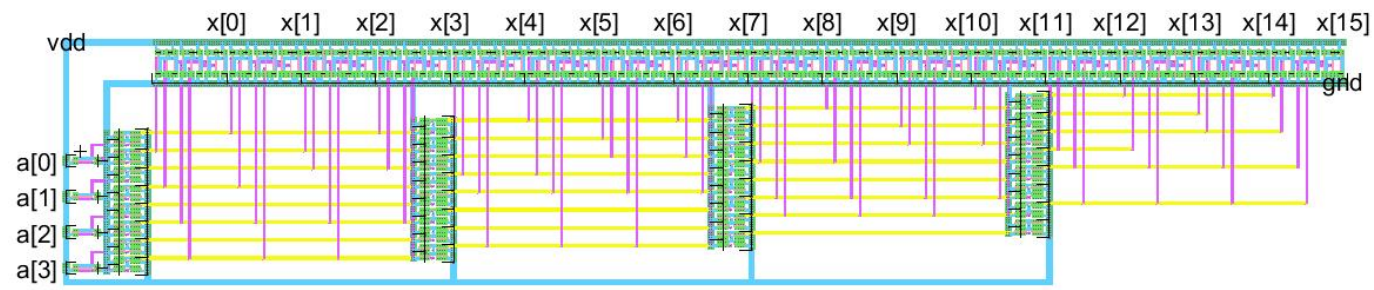

Fig. 3.4.3 Decoder Layout 


\subsubsection{Verification}

Simulated waveforms for the one-cold decoder are displayed in Fig. 3.4.4. The waveforms are plotted in a single pane to improve visibility. The ' $\mathrm{V}(\mathrm{a}[0])+80$ ' signal denotes the input vector where the first Low half cycle at 0 ns represents 0000 , High at 8 ns indicates 0001 , and so on up to 1111 at $80 \mathrm{~ns}$. Each output, $\mathrm{V}(\mathrm{x}[\mathrm{N}])$, switches Low at the corresponding 4-bit input value and remains High in all other cases.

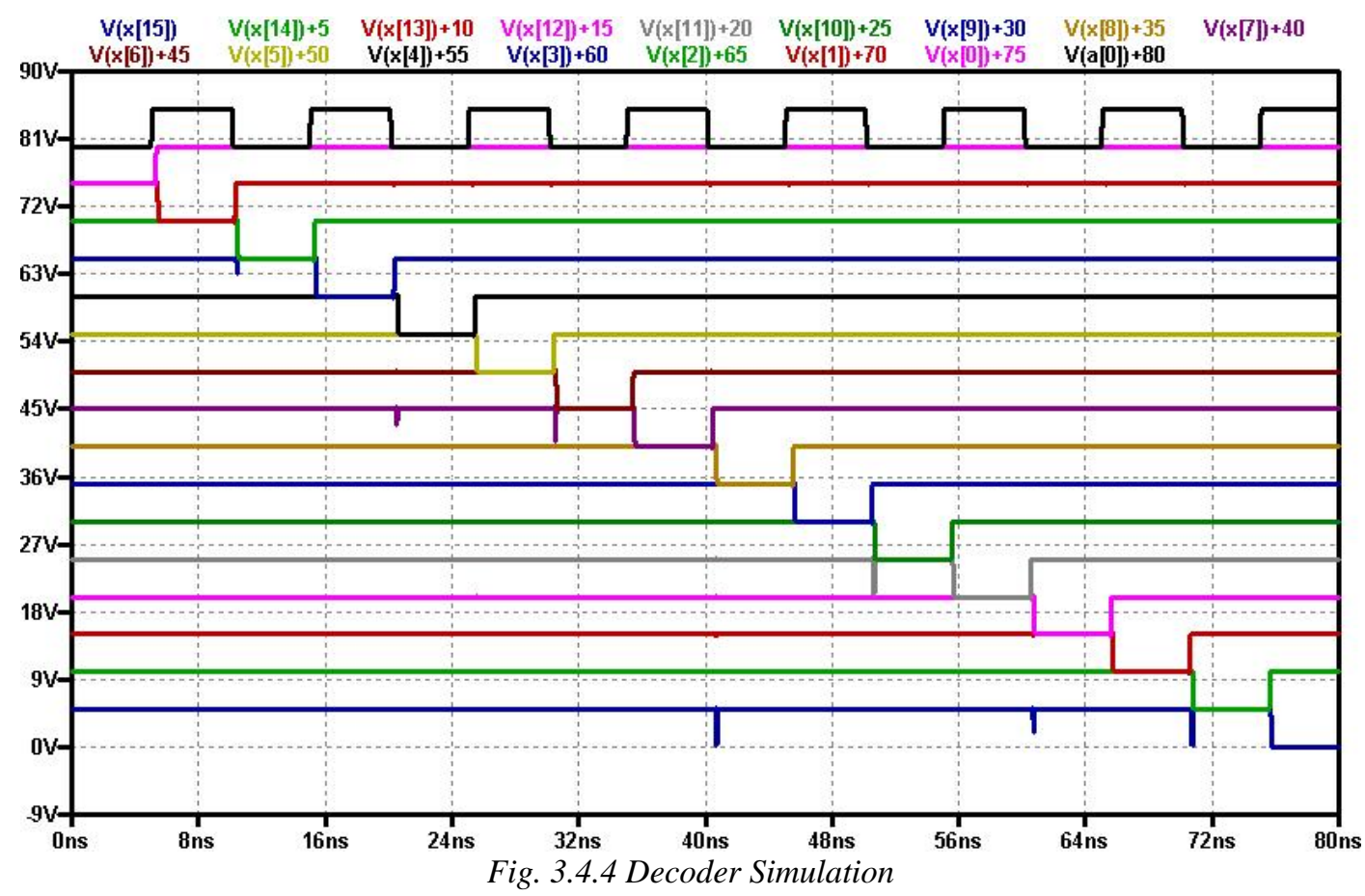




\subsection{Full Chip Design}

\subsubsection{Floorplan}

After the design and verification of the various cells and functional blocks, the next place-and-route stage incorporates the pad ring. Fig. 3.5.1 shows how the blocks are arranged within the pad ring, based on the system block diagram in Fig. 3.1.1. Input signals are routed from the left half of the chip while output signals are directed to the right, top and bottom half.

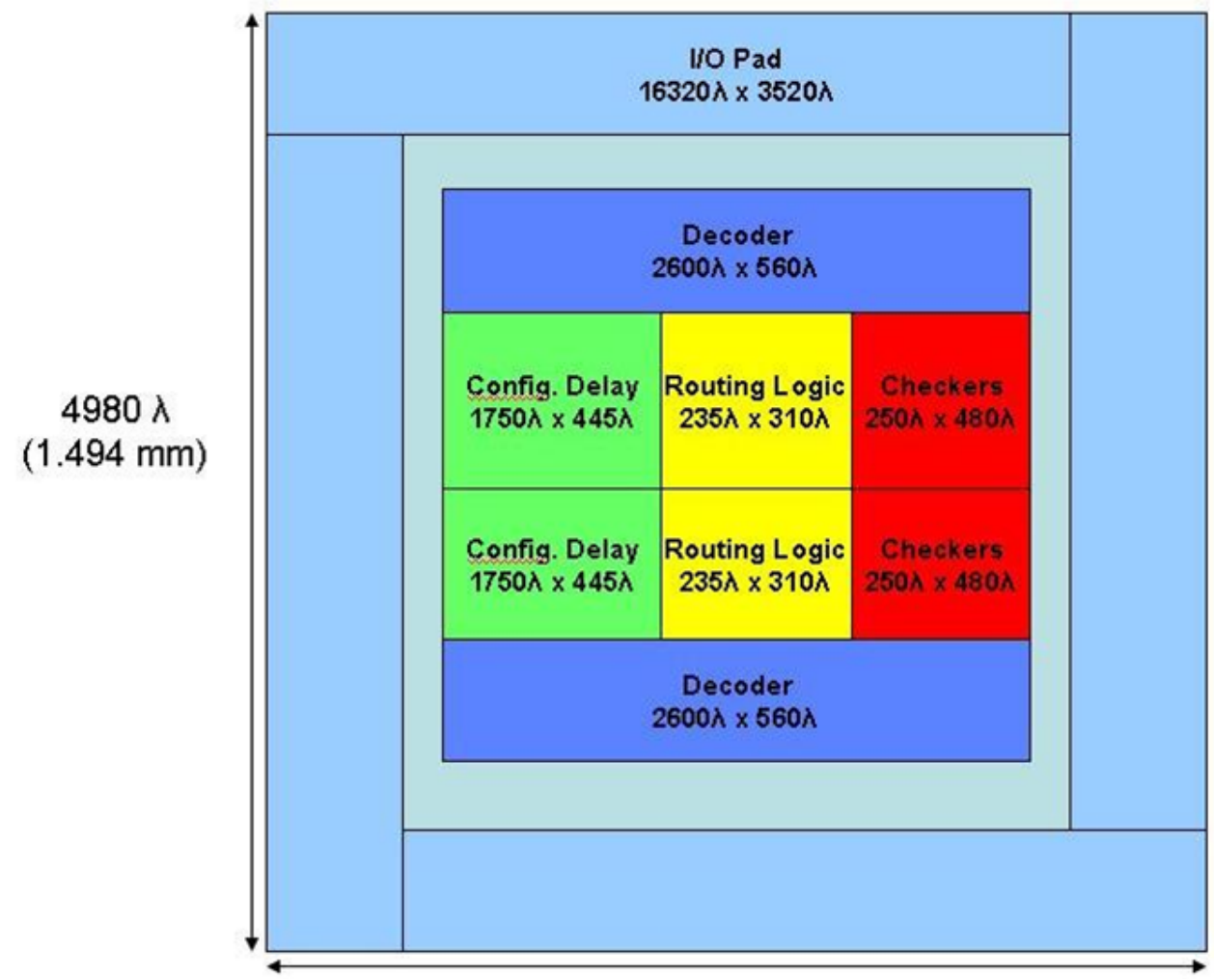

Fig. 3.5.1 Chip Floorplan

\subsubsection{Place and Route}

The pad ring was obtained from [34] as an Electric library file. It contains pads for $\mathrm{V}_{\mathrm{DD}}$, ground, analog signals and input/output ( $\left.\mathrm{I} / \mathrm{O}\right)$ (bidirectional) digital signals. $\mathrm{A}$ 
system schematic containing all modules except the pad ring was constructed (Fig. 3.5.2). This schematic includes routing logic which consists of multiplexers that switch signal sources for the checkers. The multiplexers were designed to pass the Clock/Data from the delay chain to the checker array when their common control input is Low. Setting a High to the multiplexer control input passes the external Clock/Data signal through to the checker array instead. The entire system was then implemented in layout and verified to be DRC and LVS clean (Fig. 3.5.3).

Following that, the system was routed to the pad ring in both schematics (Fig. 3.5.4) and layout (Fig. 3.5.5). Full chip layout screenshots highlighting each routing layer are included in Appendix D.

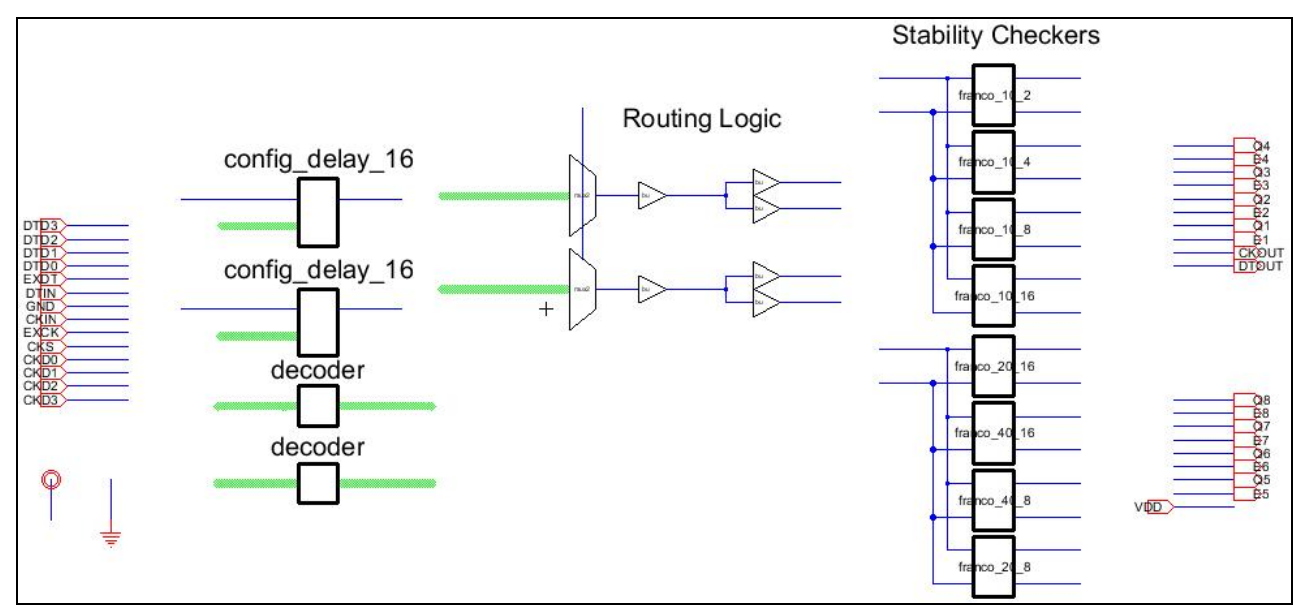

Fig. 3.5.2 System Schematic 


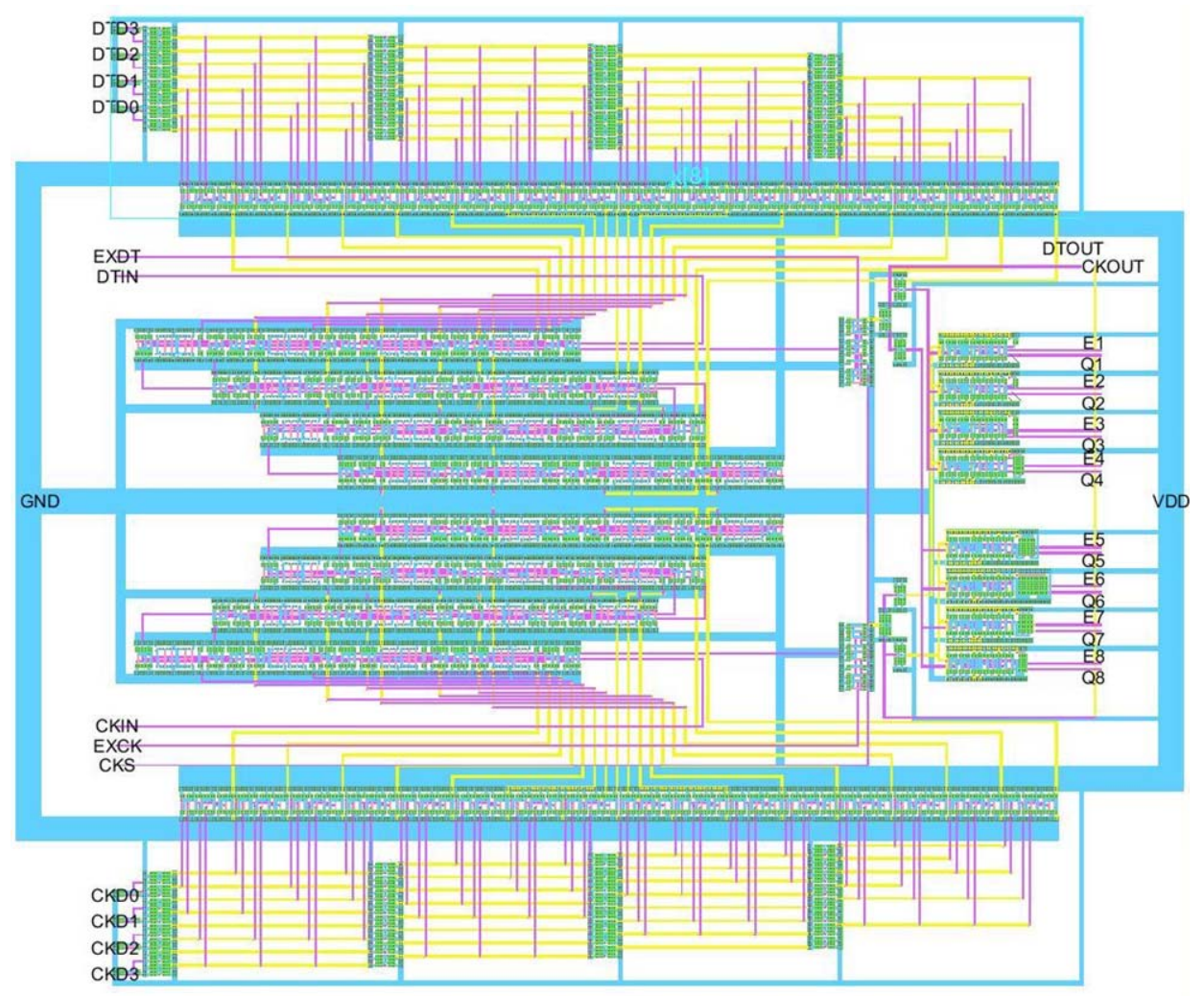

Fig. 3.5.3 System Layout

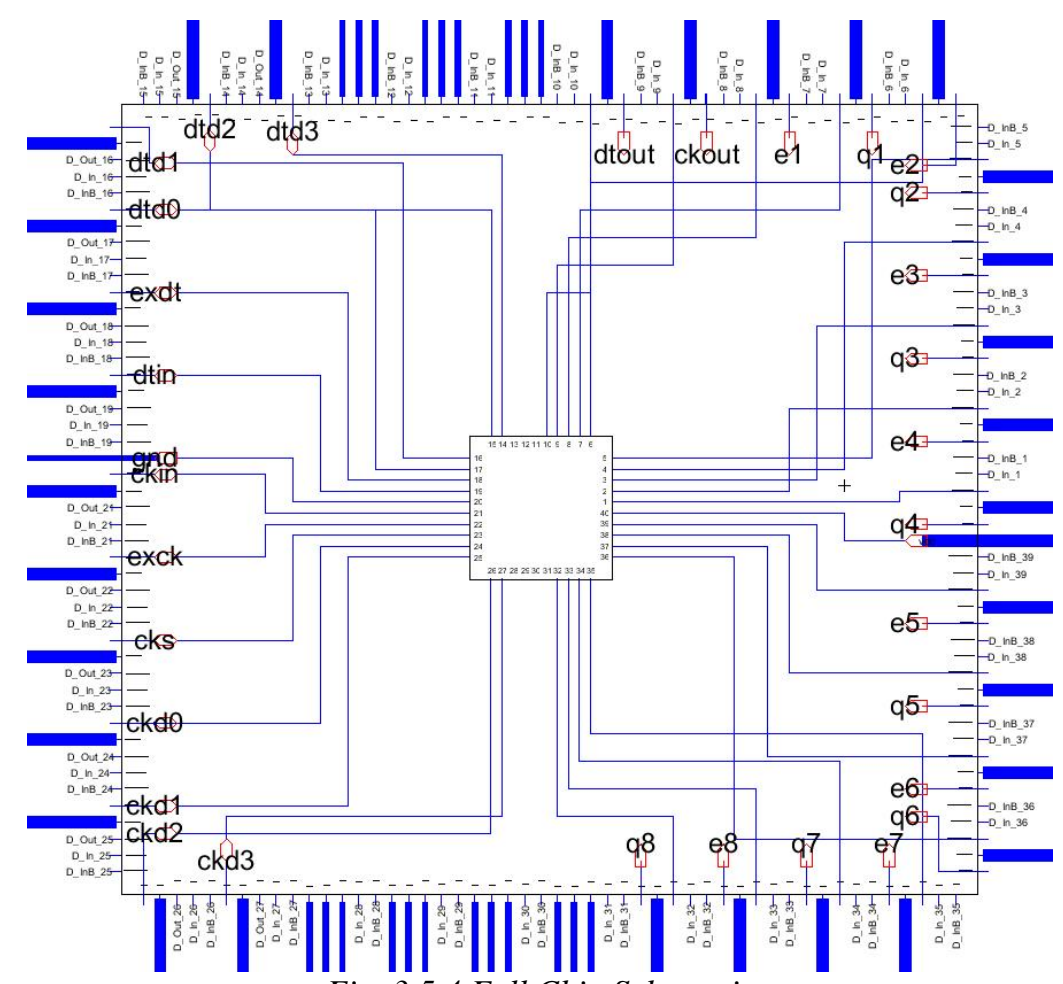

Fig. 3.5.4 Full Chip Schematic 


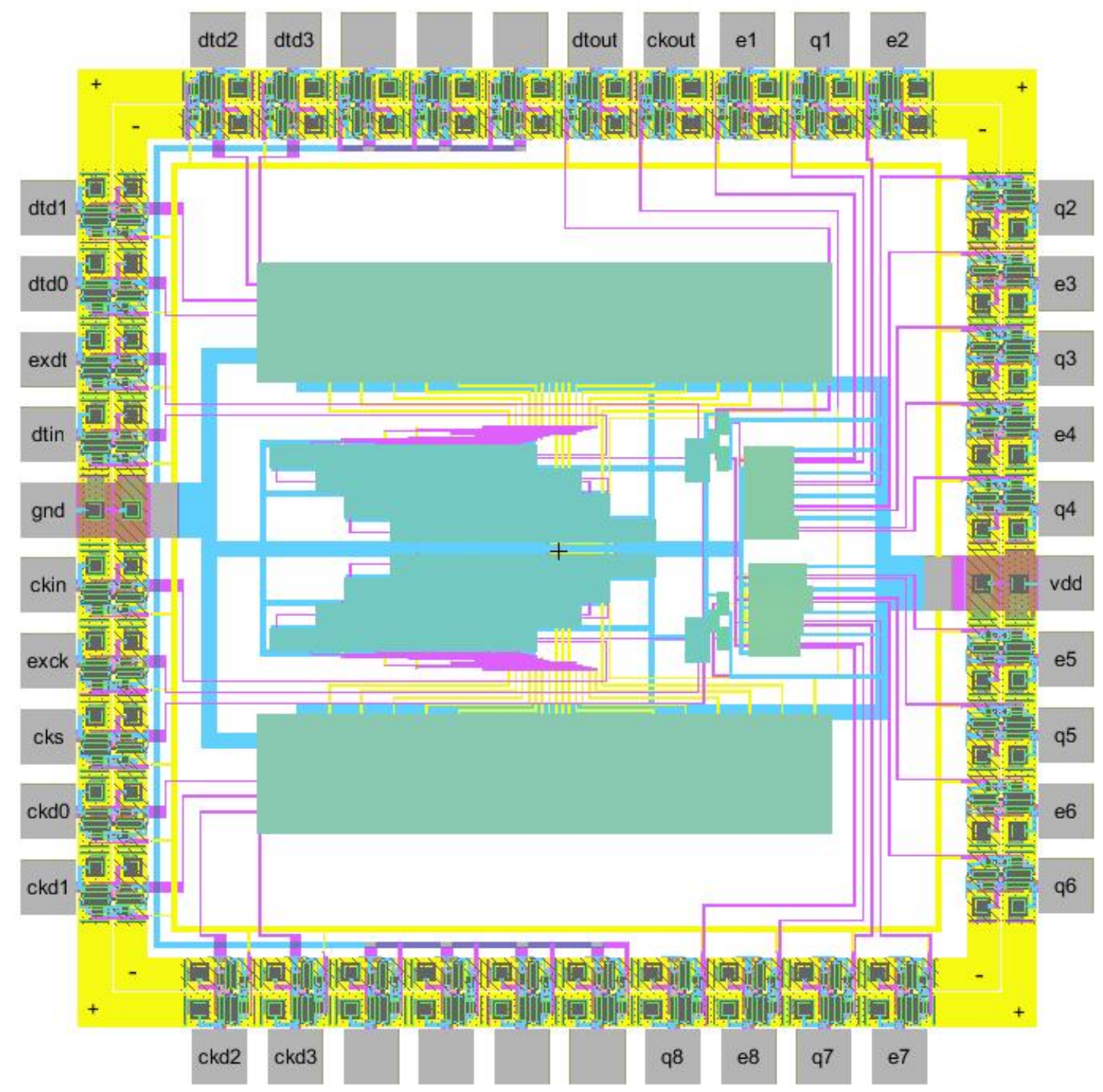

Fig. 3.5.5 Full Chip Layout

\subsubsection{Verification}

The DRC and LVS-cleaned full chip layout was then simulated to verify stability checker functionality. The configurable delays were set to generate both valid and late signals on the common data line. SPICE directives were used to generate glitches to test for instability and are listed in Appendix E. Simulations were done only to verify functionality of circuit components for this project. No timing restrictions were imposed. 


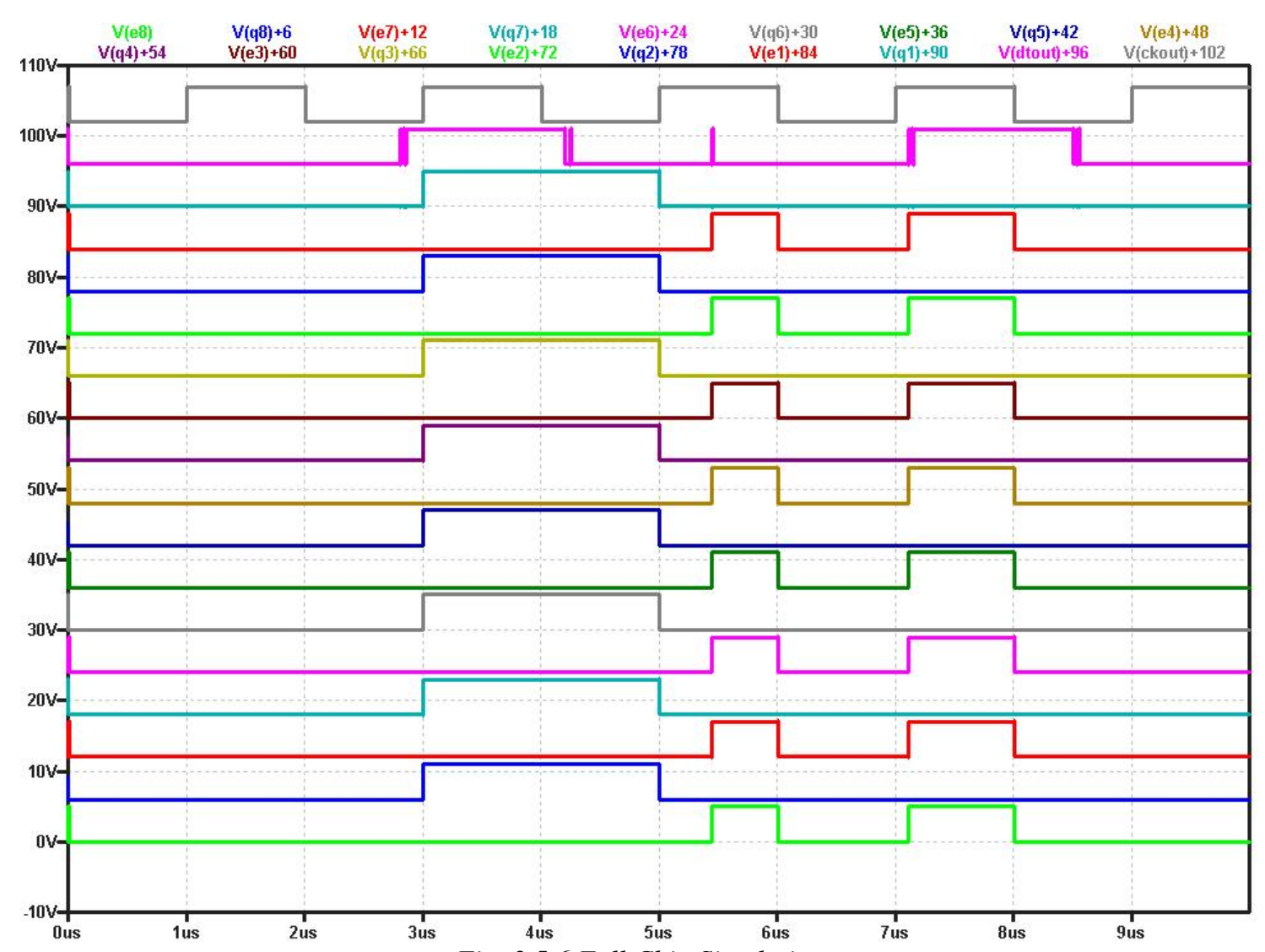

Fig. 3.5.6 Full Chip Simulation

Fig. 3.5.6 displays the waveforms of the fully responsive full chip layout design. The topmost waveform, V(ckout) denotes the common clock signal used by the checkers while the V(dtout) data shows valid data until $3 \mu$ s, a glitch at about $5.5 \mu$ s and a late signal at about $7.2 \mu \mathrm{s}$. The remaining waveforms are displayed in flip-flop output pairs, $\mathrm{V}(\mathrm{qN})$, and the error signal, $\mathrm{V}(\mathrm{eN})$, where $\mathrm{N}$ corresponds to the stability checker number as listed in Table 3.2.1. All checkers appeared to be functioning normally as flip-flops are able to detect unstable as well as late signals.

Once the chip was verified, the layout was exported to a GDS II file. The GDS II file (Graphic Database Stream) is an industry standard file format for IC layout designs. The GDS II file was taped out to MOSIS via file transfer protocol (FTP) on September 
27, 2010 for the run date scheduled the same day. MOSIS then ran a manufacturability review check to verify design syntax and layer names. MOSIS does not perform any DRC checking but examines chip dimensions, counts the pads and compares actual values with declared values.

The manufacturability review returned several warnings regarding the density of polysilicon and metal layers. Warning details are included in Appendix F. After consulting with MOSIS, the warnings were deemed irrelevant to chip functionality.

On October 11, 2010 after fulfilling all requirements, MOSIS updated the account page and sent confirmation email. This tapeout confirmation is included in Appendix G. MOSIS also provided the initial bonding diagram (Appendix H) used in test planning. The project was scheduled for the next fabrication run on November 29, 2010 and was estimated to be completed on, March 30, 2011. 


\section{CHAPTER 4}

\section{Post-Silicon Verification}

This chapter presents results on various tests conducted on the completed parts. Section 4.1 covers manufacturing issues while section 4.2 presents photographs of the completed parts. The remaining sections describe test procedures and functional verification results. Section 4.3 summarizes the test setup and equipment, while section 4.4 describes the power-up test, which checks for internal short circuits. Section 4.5 details the clock test, which verifies the straight-through routing trace of both clock and data lines. All chips passed section 4.4 and 4.5 tests. Section 4.6 presents details on the configurable delay test for Chip 1. Delay measurements for each mode were recorded and compared to SPICE simulations for analysis. Finally, section 4.7 describes stability checker tests, which verify flip-flop operation as well as glitch and late signal detection. All but Chip 7 demonstrated full functionality with respect to SPICE simulations. Details on the defect on Chip 7 are included at the end of this chapter.

\subsection{Manufacturing Issues}

MOSIS reported a fabrication failure on May 5, 2011 caused by high VIA2 resistance. The notification email sent by MOSIS (Appendix I) informed all customers about the fabrication delay and the scheduled rerun in July.

In addition to the fabrication glitch, a pad metal anomaly was reported by the packing vendor. The anomaly was claimed to affect some packaged parts in the V0BL 
run. After a series of electrical and mechanical tests completed by another customer, it was found that the affected parts met military standard MIL-STD-883 specifications. The customer concluded that all parts with the anomaly are acceptable in their critical application, allowing MOSIS to ship all parts back to its customers. Details of this issue are included in Appendix $\mathbf{J}$.

The completed parts arrived on July 7, 2011. The 10 DIP chips were shipped in packaging tubes while the 30 unpackaged dice were packed in a separate electrostatic discharge (ESD) protected case. The final bonding diagram was shipped with the parts and is included in Appendix $\mathbf{K}$.

\subsection{Visual Inspection}

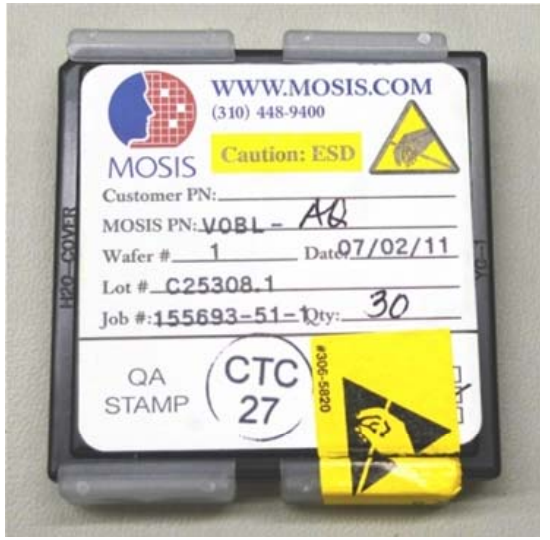

(a)

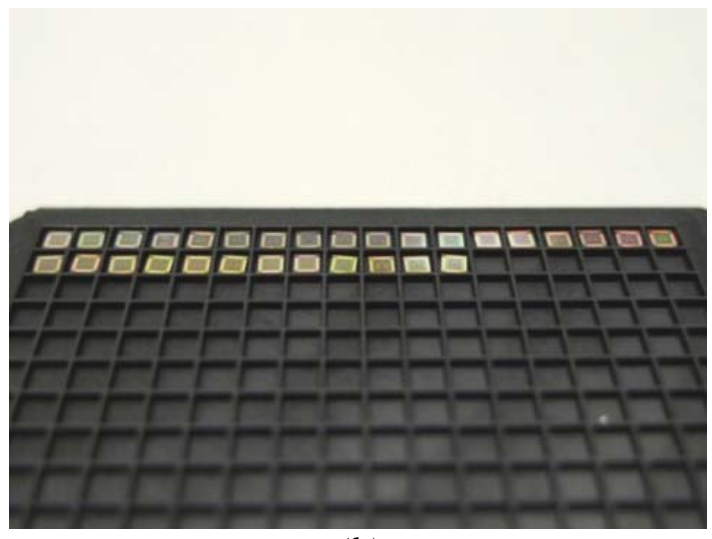

(b)

Fig. 4.2.1 Unpackaged Dice (a) ESD protected shell (b) Dice housing

The 30 unpackaged dice were housed in a plastic shell (Fig. 4.2.1 (a)) and each placed in a grid of square cavities, Fig. 4.2.1 (b). Fig. 4.2.2 shows a series of photographs of a packaged chip. The bonded dice were mounted in an open cavity package and covered by a removable ceramic plate. 


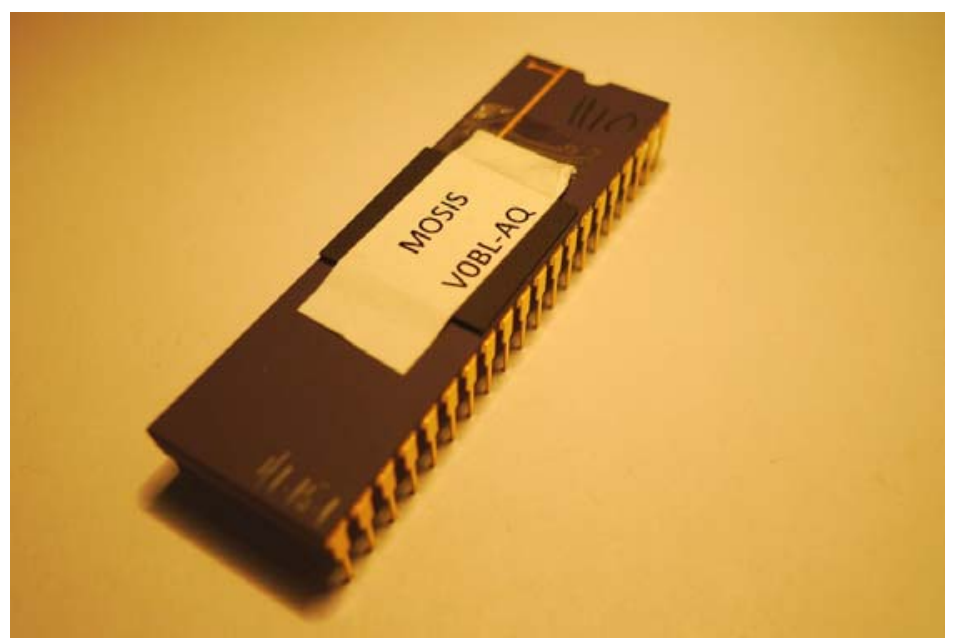

(a)

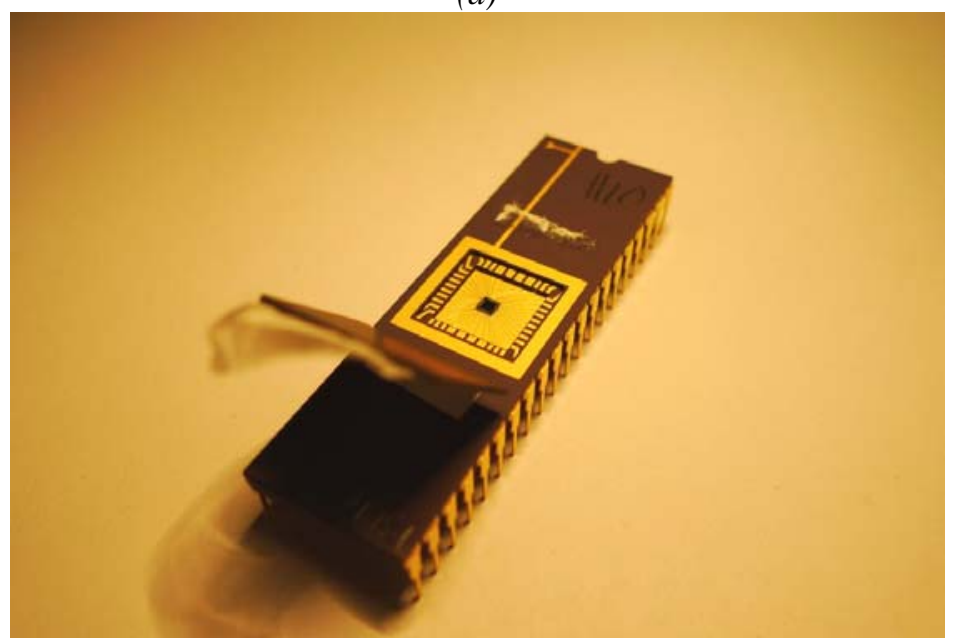

(b)

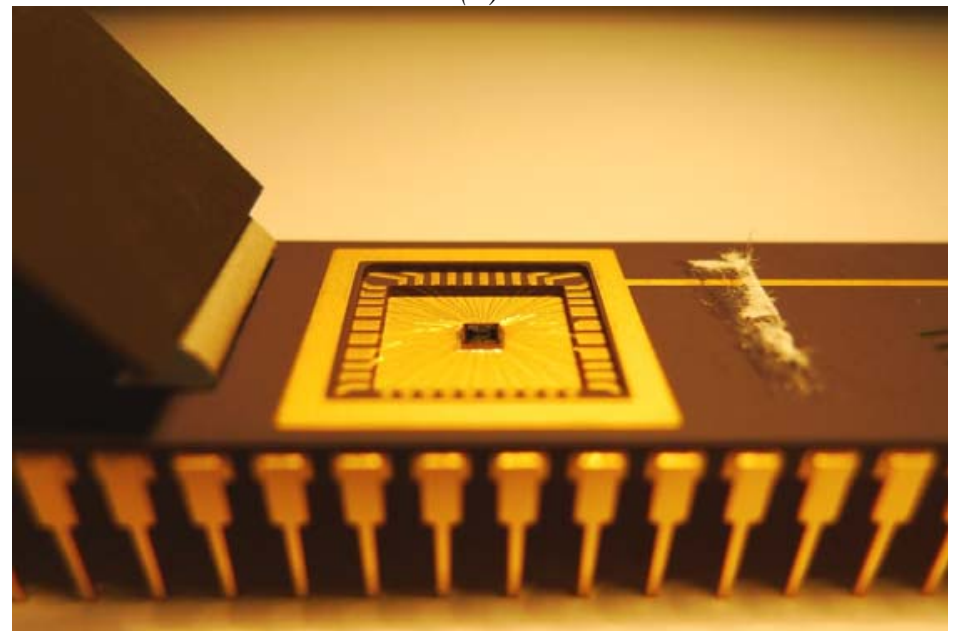

(c)

Fig. 4.2.2 Packaged Chip (a) Sealed Package (b) Exposed Package Cavity (c) Close-up View of Cavity 
Photographs of bare dice were taken through a microscope in the microfab lab in Building 41. Fig. 4.2.3 and Fig. 4.2.4 show photos taken at 20x magnification. Visual measurements on die size and on-chip structures were taken using the microscope image acquisition software and are included in Appendix L. Compared to the taped-out dice dimensions, $2.217 \mathrm{~mm}^{2}(1490 \mu \mathrm{m} x, 1490 \mu \mathrm{m})$, the finished cut die was approximately $2.551 \mathrm{~mm}^{2}(1560 \mu \mathrm{m} \times 1635 \mu \mathrm{m})$, about $15 \%$ of additional area containing MOSIS inscriptions and scribe lines.

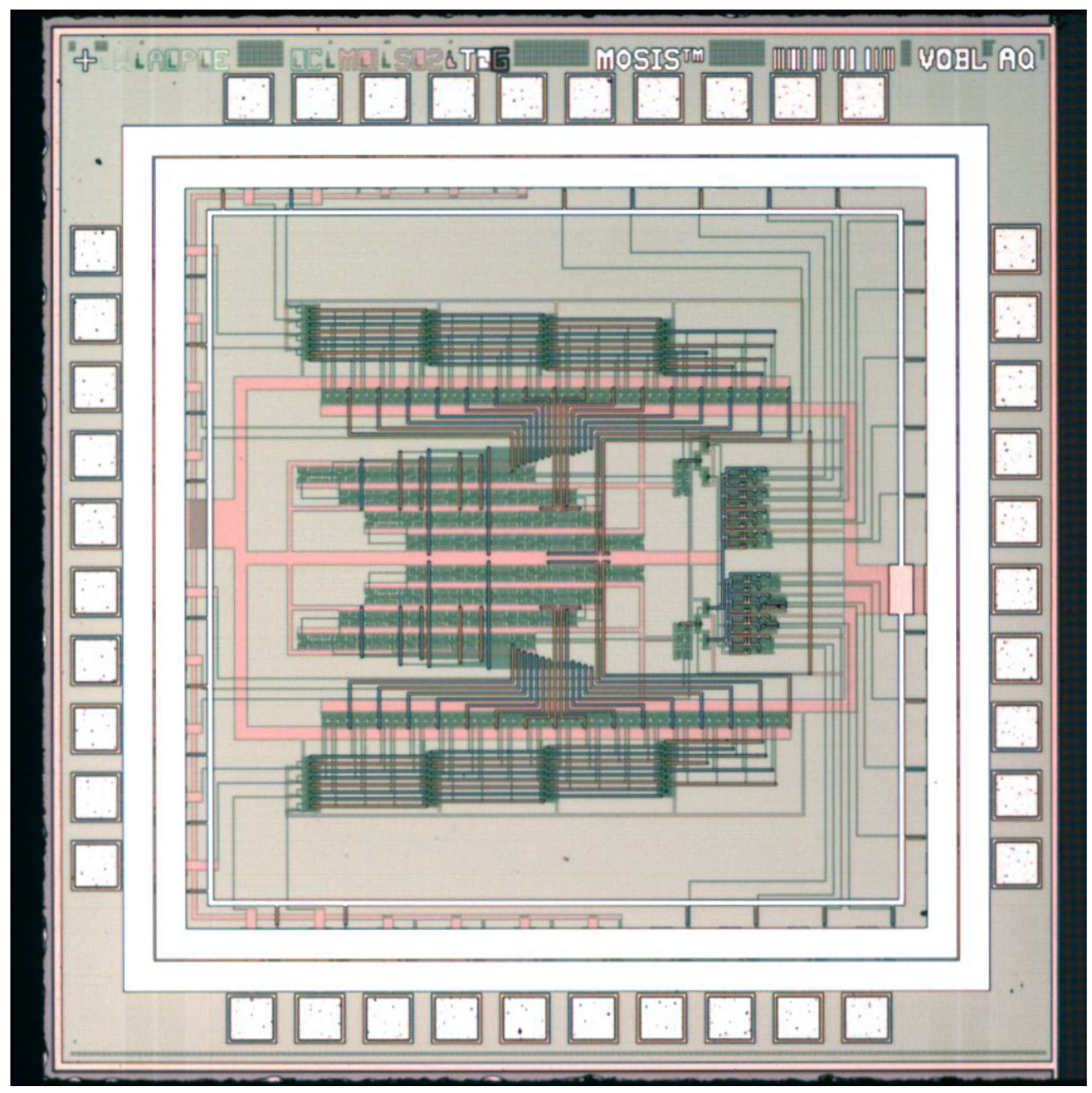

Fig. 4.2.3 Die Photograph 


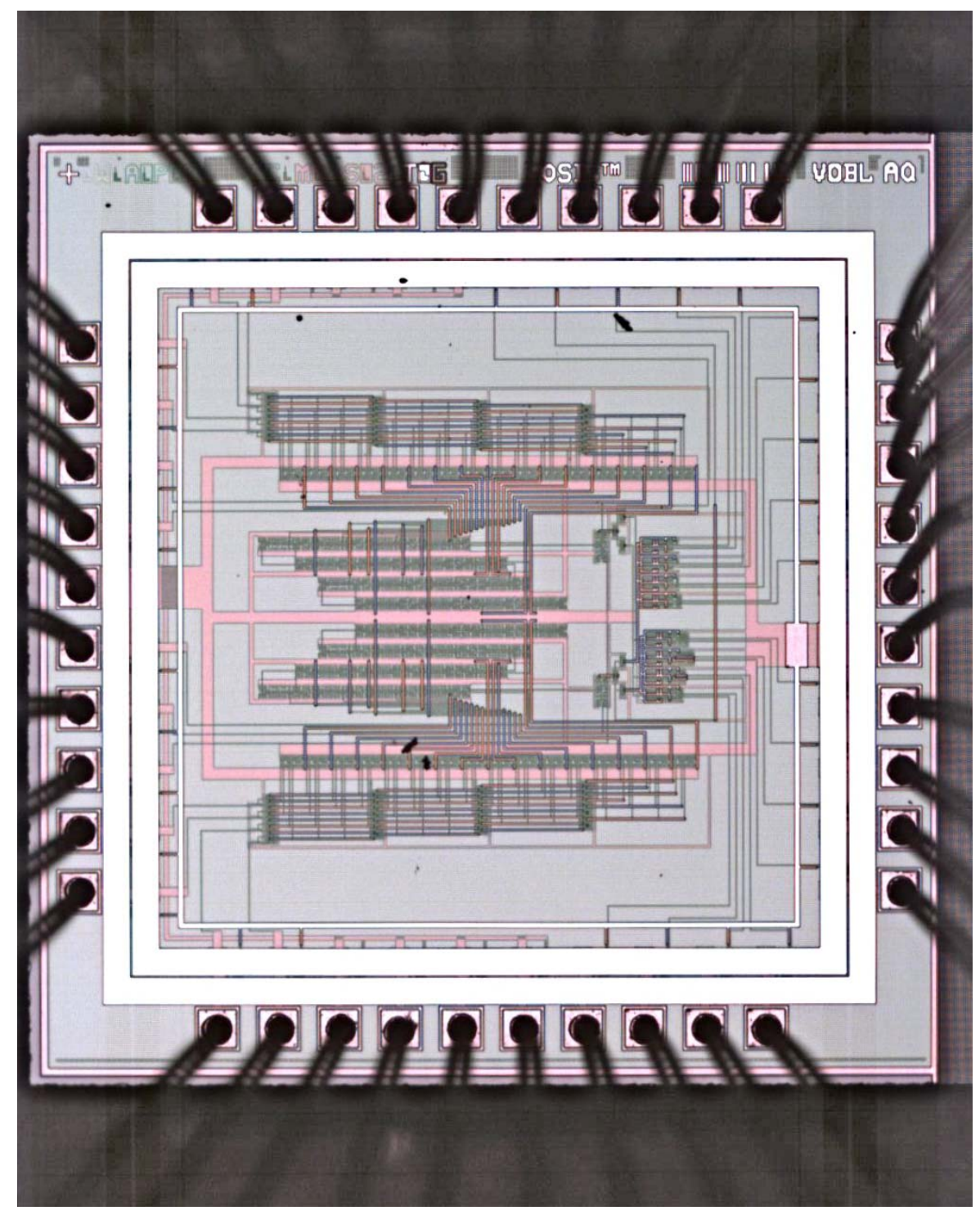

Fig. 4.2.4 Wire Bonded Die

\subsection{Test Procedures}

The 10 packaged chips were verified through four tests:

- Power-up

- Clock

- Configurable Delay

- Flip-flop and Stability Checker 
The tests require a logic analyzer to observe and capture output and error signals. Additionally, two separate function generators are required to assert test patterns at the clock and data inputs of the stability checkers. Table 4.3.1 lists the test equipment used.

\begin{tabular}{|l|l|l|}
\hline \multicolumn{1}{|c|}{ Equipment } & \multicolumn{1}{c|}{ Model } & \multicolumn{1}{c|}{ Function } \\
\hline Power Supply & Agilent E3630A & Supplies 5V V DD to test chip \\
\hline Function Generator & Agilent 33120A & Generates Clock \\
\hline \multirow{2}{*}{ Mixed Signal Oscilloscope } & Agilent MSO-X 3012A & Generates Data patterns \\
\cline { 3 - 3 } & & Logic Analyzer samples output \\
\hline
\end{tabular}

Table 4.3.1 Test Equipment

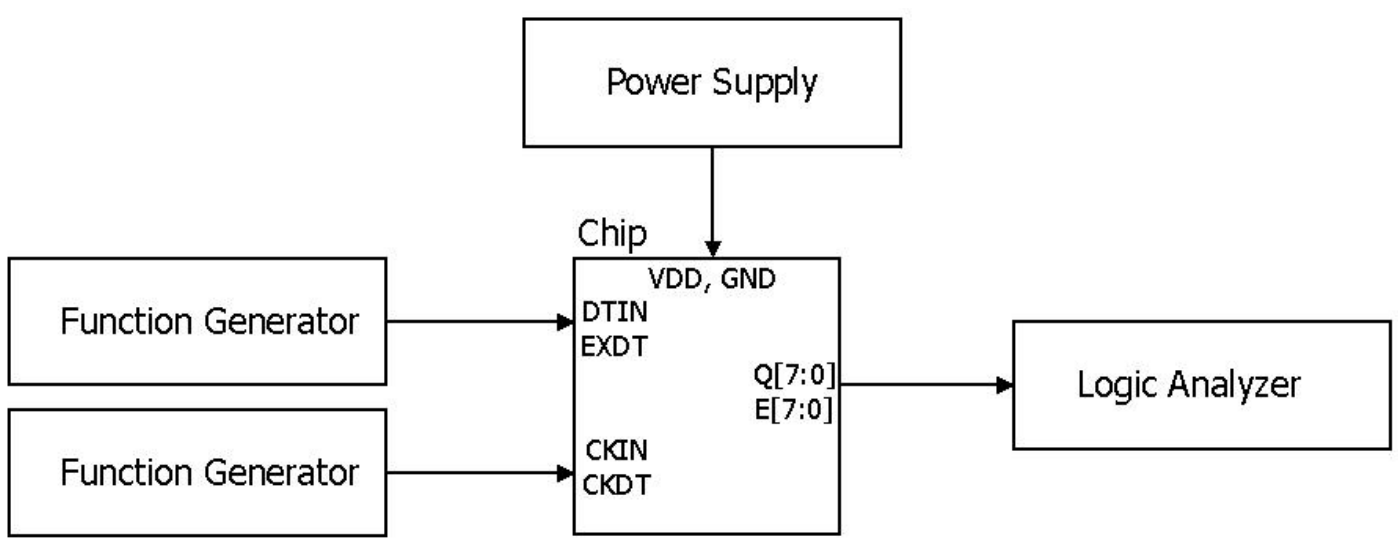

Fig. 4.3.1 Block Diagram of Test Setup

Referring to the block diagram in Fig. 4.3.1, 2 separate signals were required to provide the Clock and Data test patterns to the chip. The Clock was generated by the Agilent 33120A Function Generator while the Agilent MSO-X 3012A Mixed Signal Oscilloscope was used as to generate the Data signal. The Mixed Signal Oscilloscope also functioned as a logic analyzer to observe and capture test vectors and output signals. 


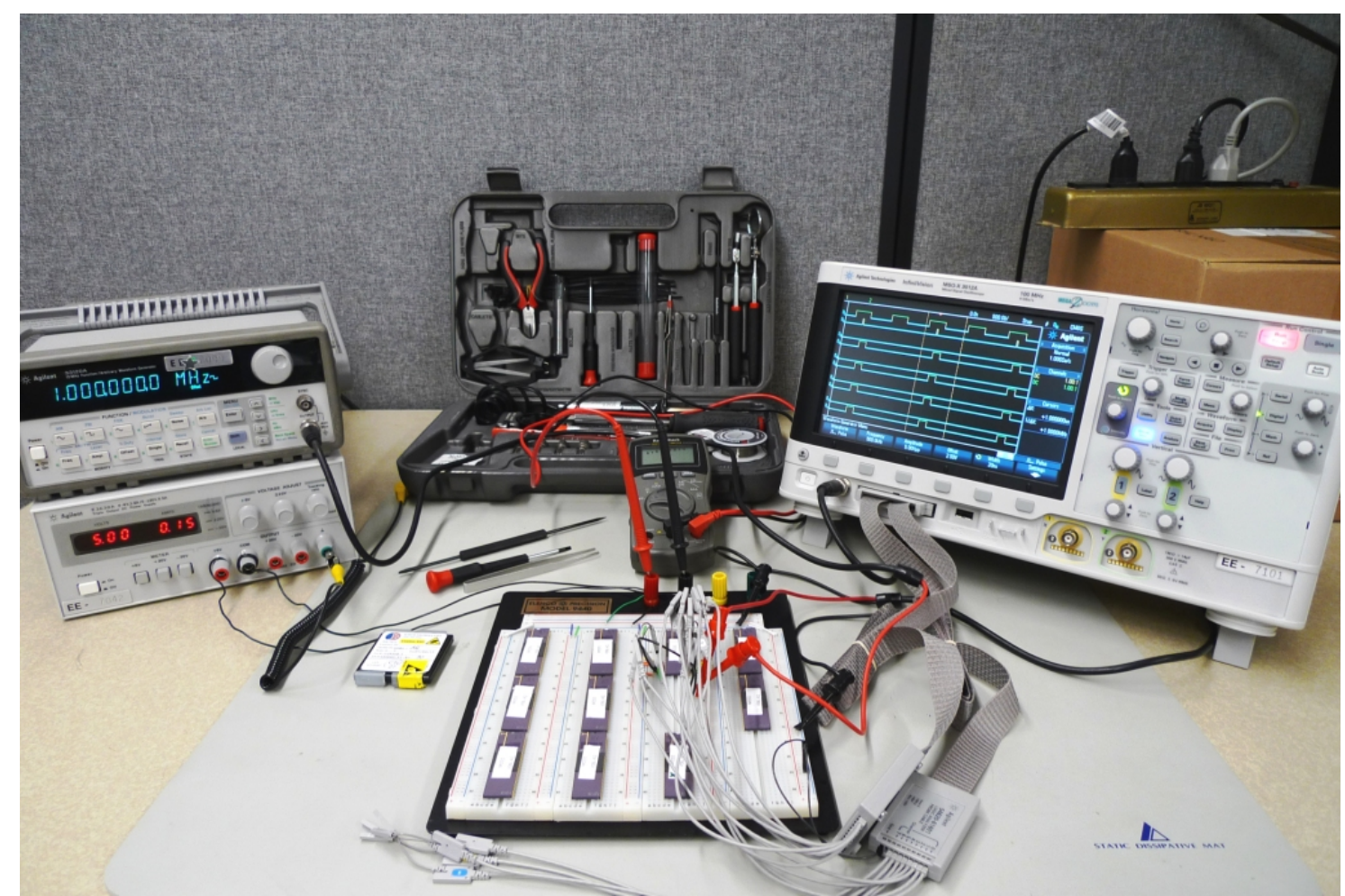

Fig. 4.3.2 Test Setup.

Fig. 4.3.2 shows the entire test setup with the power supply and function generator on the left and mixed signal oscilloscope on the right side of the photo. The test chip was mounted on a breadboard and connected according to the pinout illustrated in Appendix M. Table 4.3.2 describes pin labels and functions while Fig. 4.3.3 shows a closeup view of the logic probe connections for stability checker testing. All chips were assigned a number from 1 to 10 for testing.

The output pair [CKOUT, DTOUT] displays the common Clock and Data signals routed to the stability checker array. These signals are selected by applying the appropriate logic level to the CKS input, which is the select line of the multiplexers described in section 3.5.2. CKS selects between the internally delayed signal pair, [CKIN, DTIN], and the external signal pair [EXCK, EXDT]. 


\begin{tabular}{|c|c|l|}
\hline Pin Name & Type & \multicolumn{1}{|c|}{ Description } \\
\hline EXCK & Input & Clock without delay \\
\hline EXDT & Input & Data without delay \\
\hline CKIN & Input & Clock to delay chain \\
\hline DTIN & Input & Data to delay chain \\
\hline CKS & Input & Selects signal source \\
\hline CKOUT & Output & Clock to checkers \\
\hline DTOUT & Output & Data to checkers \\
\hline CKD[3:0] & Input & Selects magnitude of delayed clock \\
\hline DTD[3:0] & Input & Selects magnitude of delayed data \\
\hline Q[7:0] & Output & Flip-flop output \\
\hline E[7:0] & Output & Stability checker error indicator \\
\hline
\end{tabular}

Table 4.3.2 Pin Descriptions

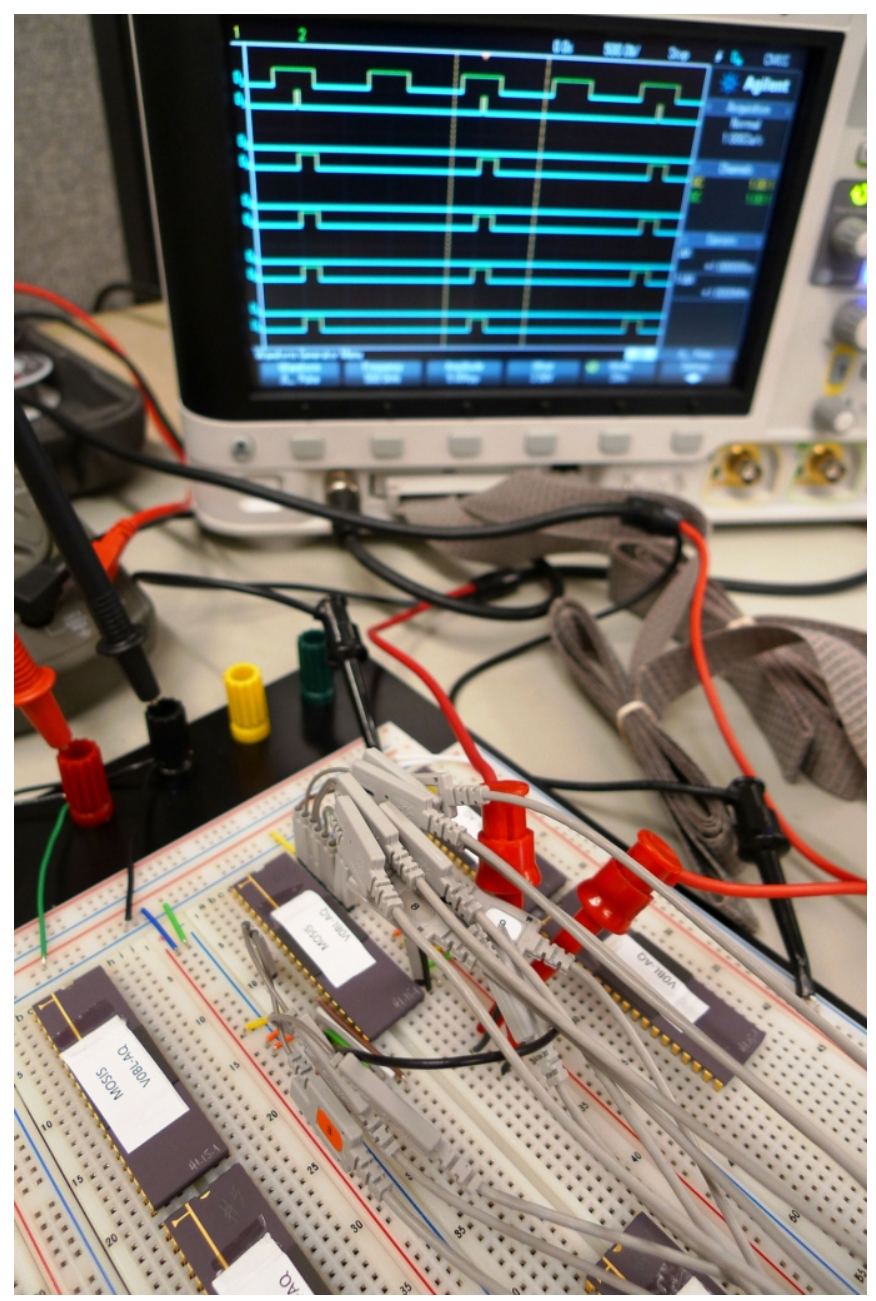

Fig. 4.3.3 Test Chip Setup 
Inputs CKD and DTD are 4-bit configurable delay chain control vectors for the Clock and Data signals, respectively. These signals were connected directly to the power rails during test.

Finally, the Q[7:0] and E[7:0] outputs are flip-flop outputs and checker error indicator lines, respectively. The vector numbering of each output pin corresponds to the stability checker labels listed in Table 3.2.1, i.e.: Q[0] and E[0] are outputs of SD0.

\subsection{Power-up Test}

\subsubsection{Test Setup}

The objective of this initial test is to detect chip defects that could cause a short circuit. All clock inputs were asserted to High including CKS, which selects [EXCK, EXDT]. Both delay chain control vectors were set to Low. Logic probes were connected to all outputs and the chip was gradually powered up $0 \mathrm{~V}$ to $\mathrm{V}_{\mathrm{DD}}$. A functional chip displays Highs at [CKOUT, DTOUT], Lows at all Q outputs since no sampling clock pulse has been asserted, and Highs at all E outputs since all checkers have not been reset.

\subsubsection{Test Results}

Fig. 4.4.1 shows a screenshot of a successful power-up test where all signals are stable and holding correct logic values. The Bus1 signal includes [DTOUT,CKOUT,CKIN,DTIN], which also corresponds to the first four signals listed at the top of the screen. 


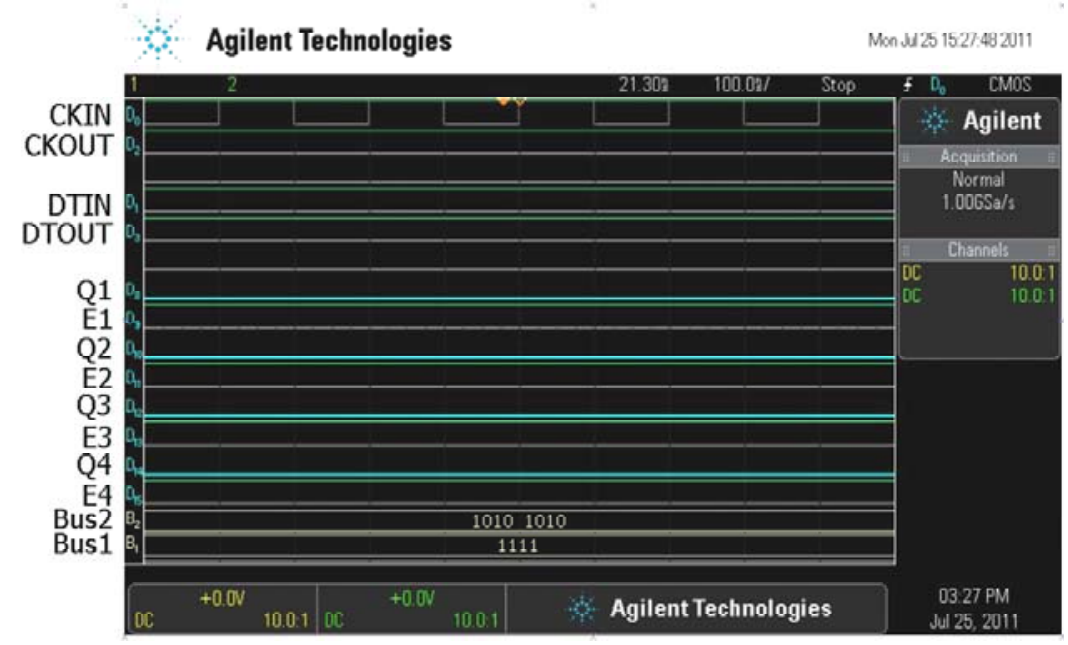

Fig. 4.4.1 Power-Up Test Waveforms

Bus2 consists of checker signal pairs listed in Table 4.4.1. All chips passed the power-up test except for a checker error on Chip 7. The E output of SD6 on Chip 7 displayed a Low unlike its counterparts. Details on this fault are discussed in section 4.7.

\begin{tabular}{|c|c|}
\hline Signal Name & Output \\
\hline Bus2[0] & Q1 \\
\hline Bus2[1] & E1 \\
\hline Bus2[2] & Q2 \\
\hline Bus2[3] & E2 \\
\hline Bus2[4] & Q3 \\
\hline Bus2[5] & E3 \\
\hline Bus2[6] & Q4 \\
\hline Bus2[7] & E4 \\
\hline \multicolumn{2}{|c}{ Table 4.4.1 }
\end{tabular}

\subsection{Clock Test}

\subsubsection{Test Setup}

This test verifies the clock routing logic by injecting waveforms into the [EXCK, EXDT] input and observing the outputs at [CKOUT, DTOUT]. CKS was set to High to 
select the appropriate clock source. A $1 \mathrm{MHz}$ square wave with 50\% duty cycle was used Both the Clock and Data lines were tested and signal propagation delay through the chip was estimated on the logic analyzer.

\subsubsection{Test Results}

Fig. 4.5.1 (b) shows successful clock test results based on SPICE simulation waveforms in Fig. 4.5.1 (a). CKOUT displays the input waveform from EXCK, in-phase and toggling at $1 \mathrm{MHz}$ as expected. Propagation delay was measured using cursors on the analyzer; approximately 4 ns on the data line (Fig. 4.5.2). All chips passed the clock test.

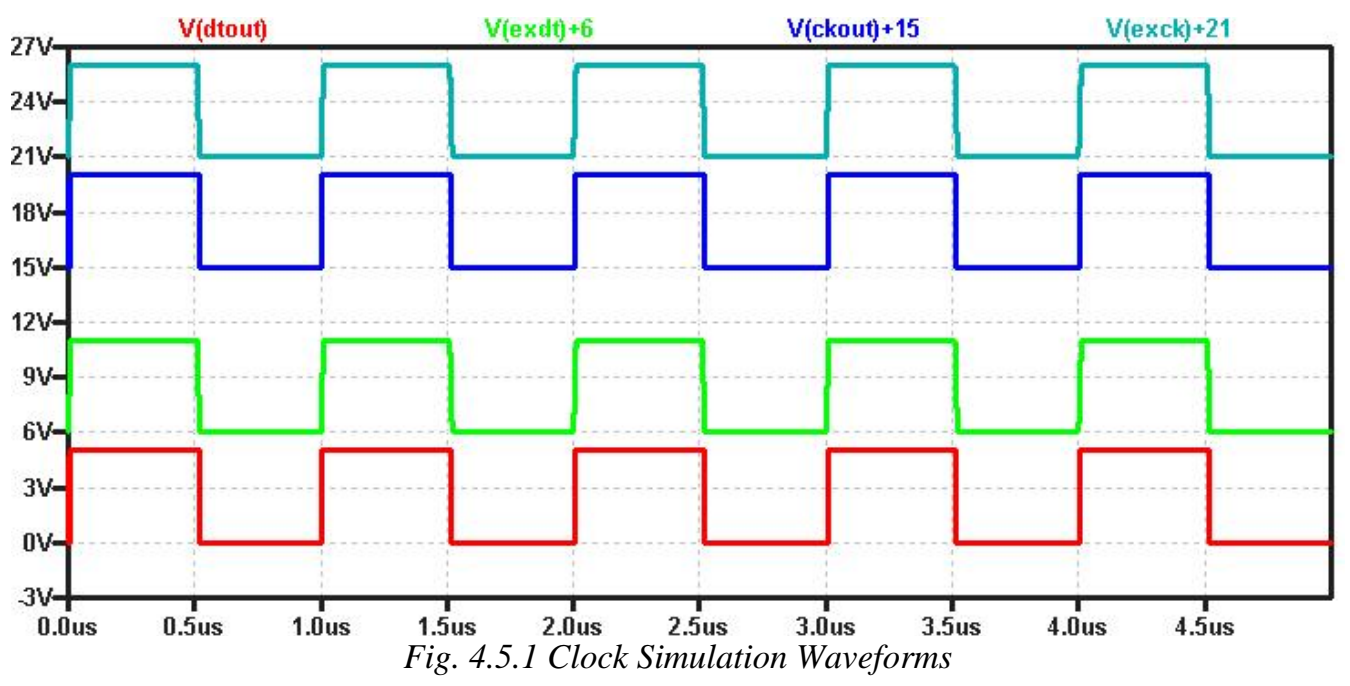




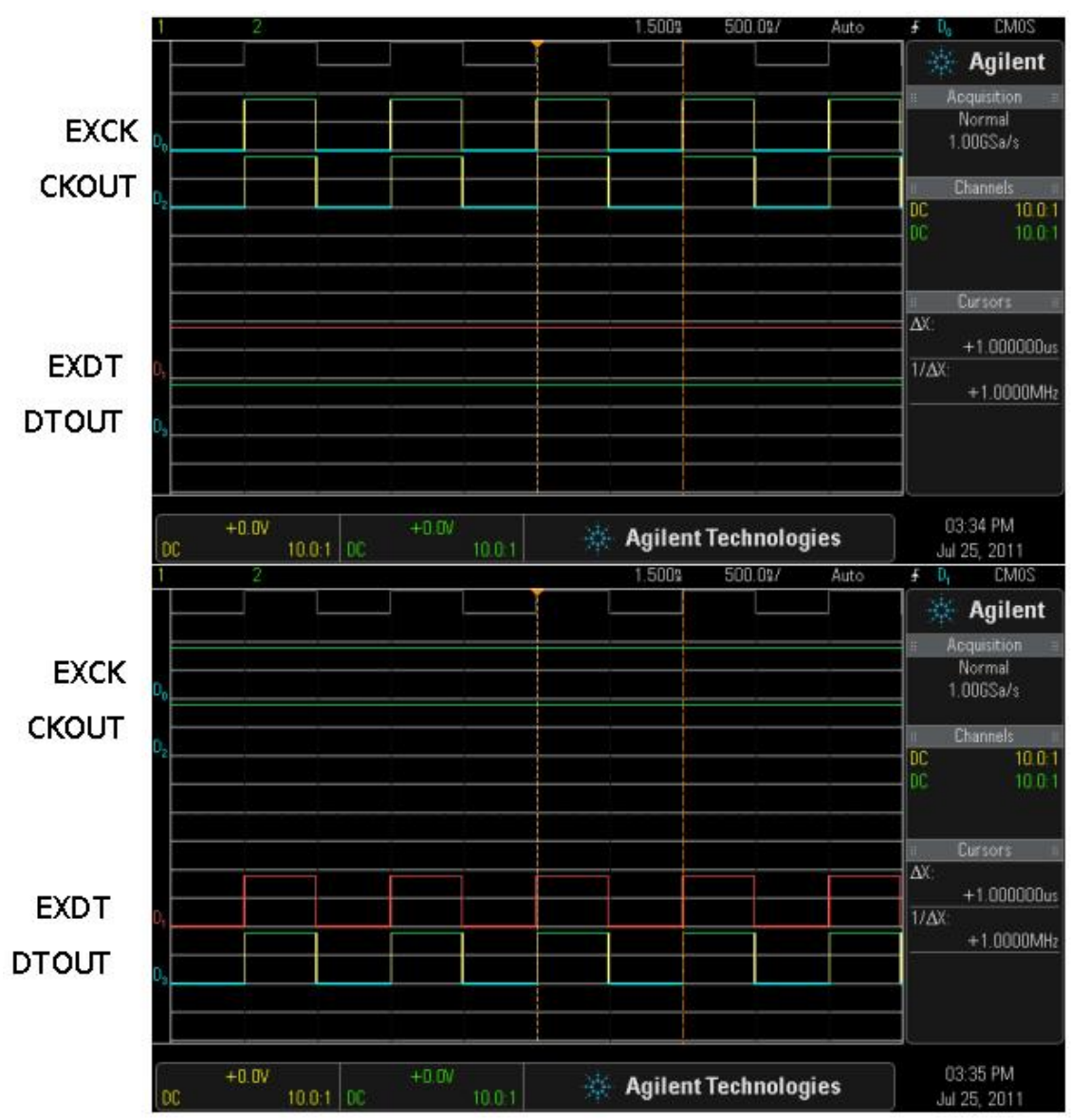

Fig. 4.5.2 Chip Clock Test Waveforms

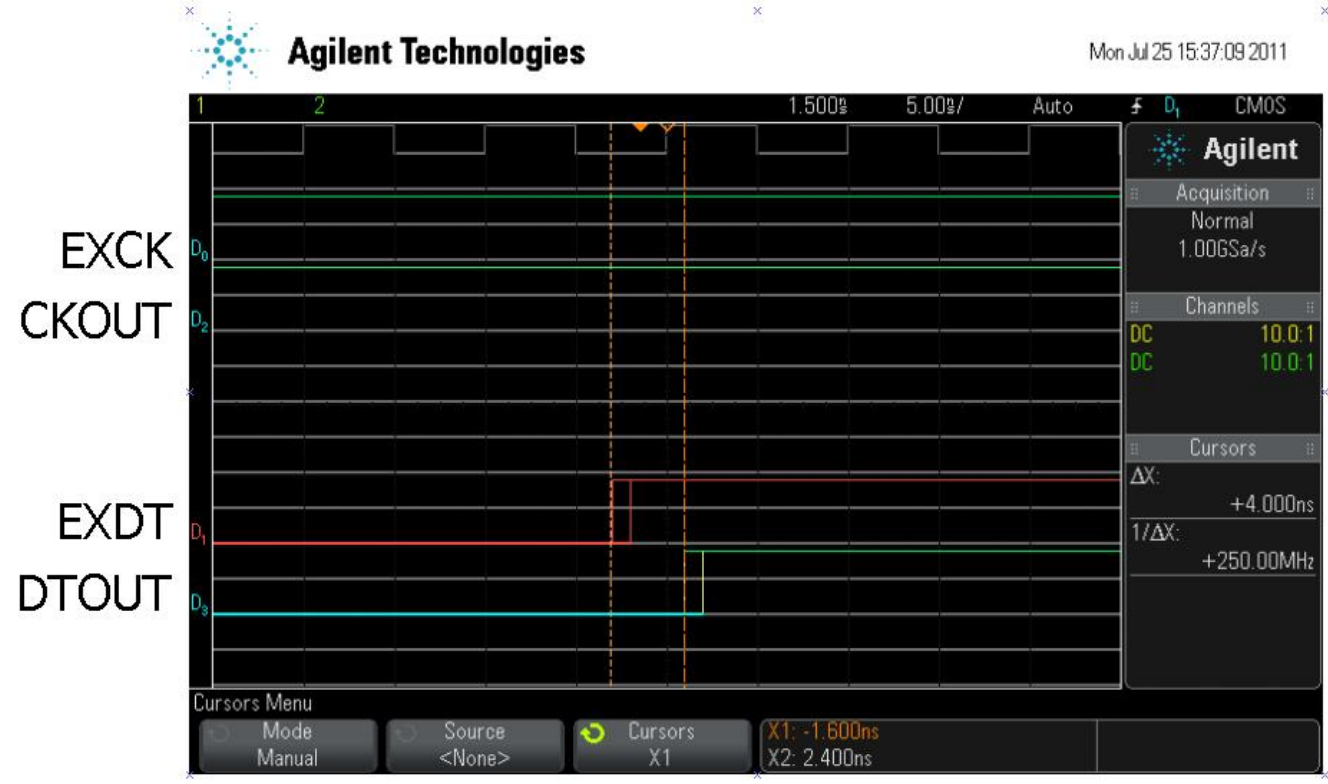

Fig. 4.5.3 Propagation Delay Measurement 


\subsection{Configurable Delay Test}

\subsubsection{Test Setup}

This test examines the delay range and precision of each configurable delay chain. The control vectors were stepped through the 16 delay stages. A $1 \mathrm{MHz}$ square wave was injected into CKIN and DTIN, and the resulting outputs were sampled at CKOUT and DTOUT, respectively. Fig. 4.6.1 shows delay measurements of the first and last delay stage for CKIN and EXCK. Delay measurements were recorded for each delay mode plotted for Chip 1 only.

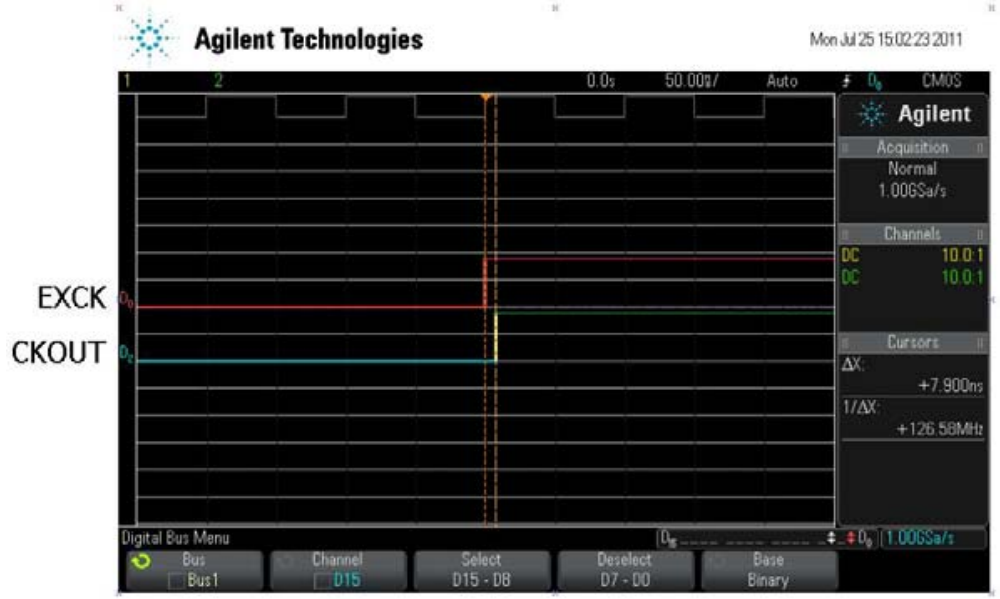

(a)

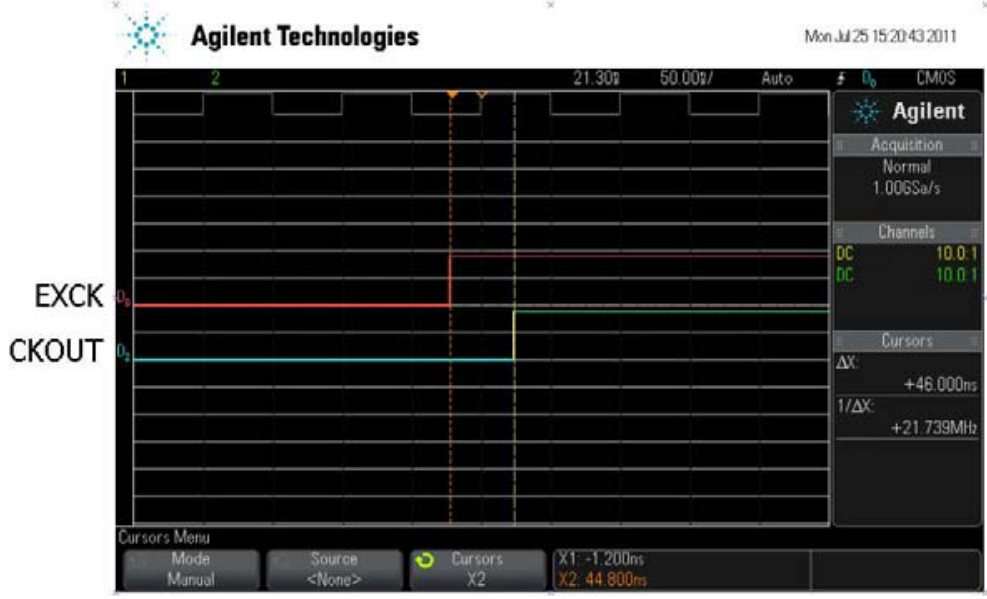

(b)

Fig. 4.6.1 Configurable Delay Waveforms (a) Delay Stage 1, Chip 1 (b) Delay Stage 16, Chip 1 


\subsubsection{Test Results}

Delay measurements for both Clock and Data lines are listed and plotted in Fig. 4.6.2. The plot shows a consistent delay increment for both delay chains relative to one another. For both delay chains, the average delay is approximately $2.5 \mathrm{~ns}$ per stage compared to SPICE simulated values of $0.7 \mathrm{~ns}$ per stage, indicating a difference greater than 3 times. Delay ranges differ by about 4 times with the simulated range being $11 \mathrm{~ns}$ and the actual measurement being $38 \mathrm{~ns}$. This difference reflects the discrepancy of timing estimates between simulation and actual values. This inaccuracy in timing estimates should be heavily considered in speed critical designs in future projects. However, results show a consistent linear delay increment for each delay stage as expected. The SPICE code used for verification is listed in Appendix N.1.

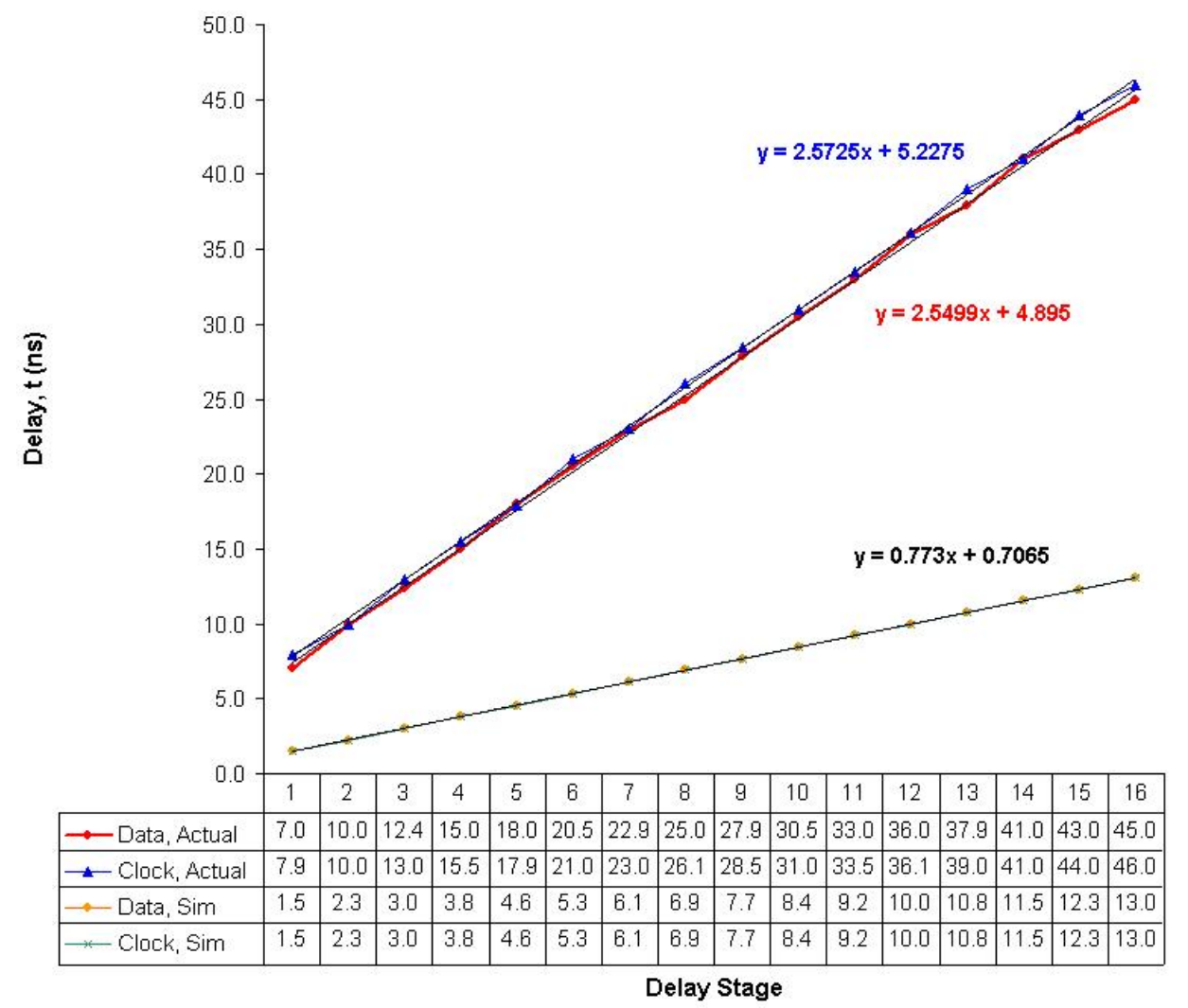

Fig. 4.6.2 Configurable Delay Measurements 


\subsection{Stability Checker Test}

\subsubsection{Test Setup}

This test verifies stability checker functionality as described in sections 3.2 .4 and 3.5.3. The checkers were tested to verify flip-flop operation, glitch detection and late signal arrival detection. All tests were conducted using a $1 \mathrm{MHz}$ sampling clock signal with an initial duty cycle of 50\%, which defines the checking period. The MSO-X 3012A was set to generate a $20 \mathrm{~ns}$ High pulse to simulate a glitch on the Data line. Late signal events were captured by executing several single sample captures on the logic analyzer, and by varying the data signal pulse width until the events were observed.

\subsubsection{Test Results}

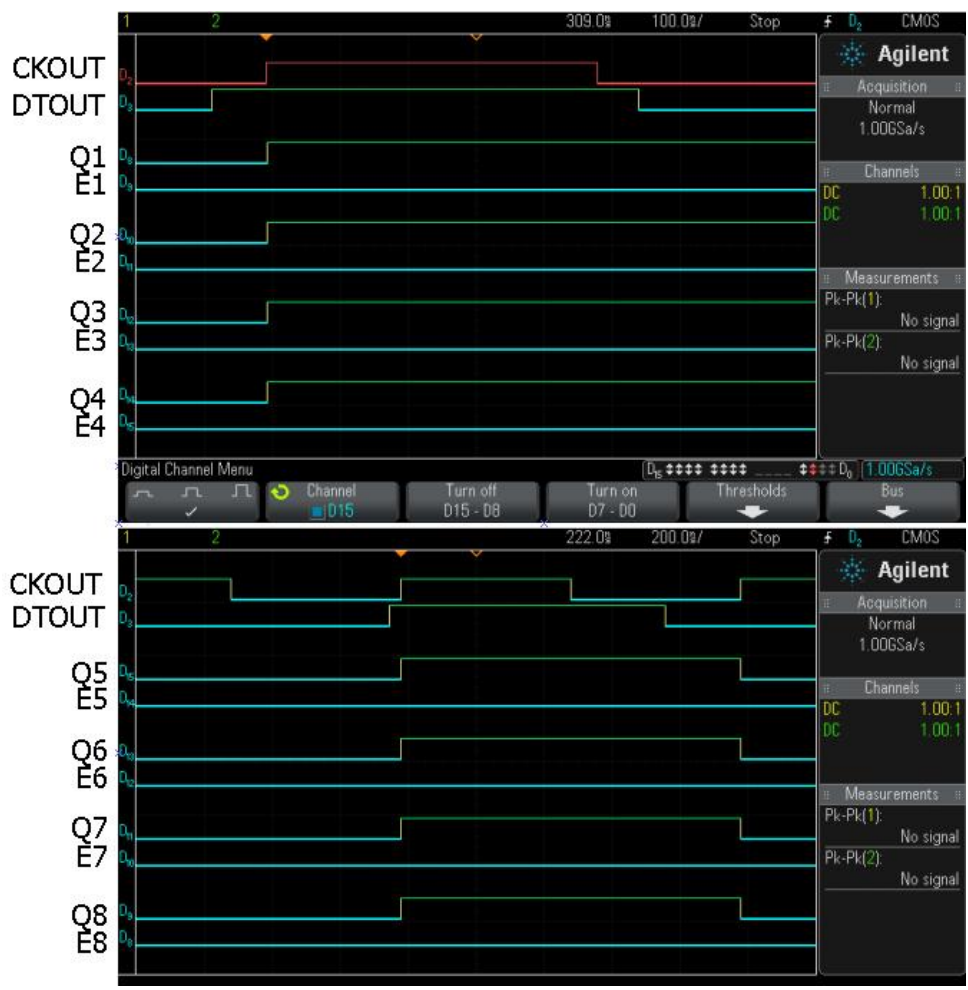

Fig. 4.7.1 Flip-flop Operation Test Waveforms 
A successful test result for flip-flop operation is shown in Fig. 4.7.1. All checkers correctly sample Data at the rising Clock edge while error signals remain inactive.

Fig. 4.7.2 displays output waveforms of a successful glitch detection test. All checkers were able to capture the 20 ns glitch on the Data line correctly, Fig. 4.7.2 (a). This result was verified against SPICE simulation waveforms in Fig. 4.7.2 (b). Appendix N.2 lists the SPICE code used to simulate this glitch.
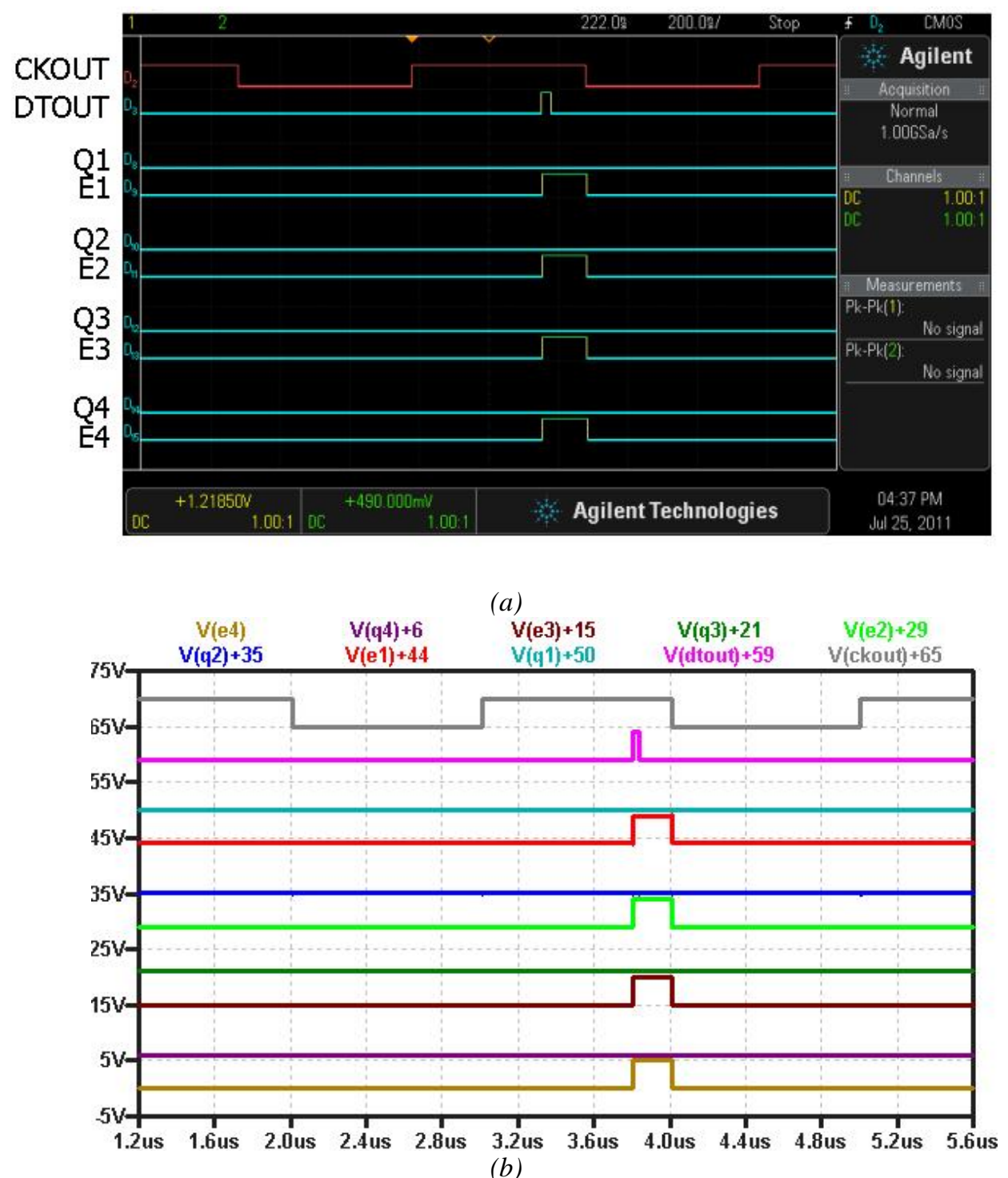

Fig. 4.7.2 Glitch Test Waveforms (a) Chip Test Waveforms (b) SPICE Simulation Waveforms 
All checkers responded as expected to simulated late signal errors, Fig. 4.7 .3 (a) based on SPICE simulations shown in Fig. 4.7.3 (b). All error signals reset correctly at the end of the checking period. The SPICE code is included in Appendix N.3.

However, a late signal occurring about 5 ns before the end of the checking period was not captured by SD6 through SD8 (Fig. 4.7.4). The larger error transistor W/ L ratios of SD6 through SD8 correspond to a larger RC delay and hence slower transition time, which in turn limits the response time of checkers with wider error transistors. This reflects on checker sensitivity for different error transistor sizes.
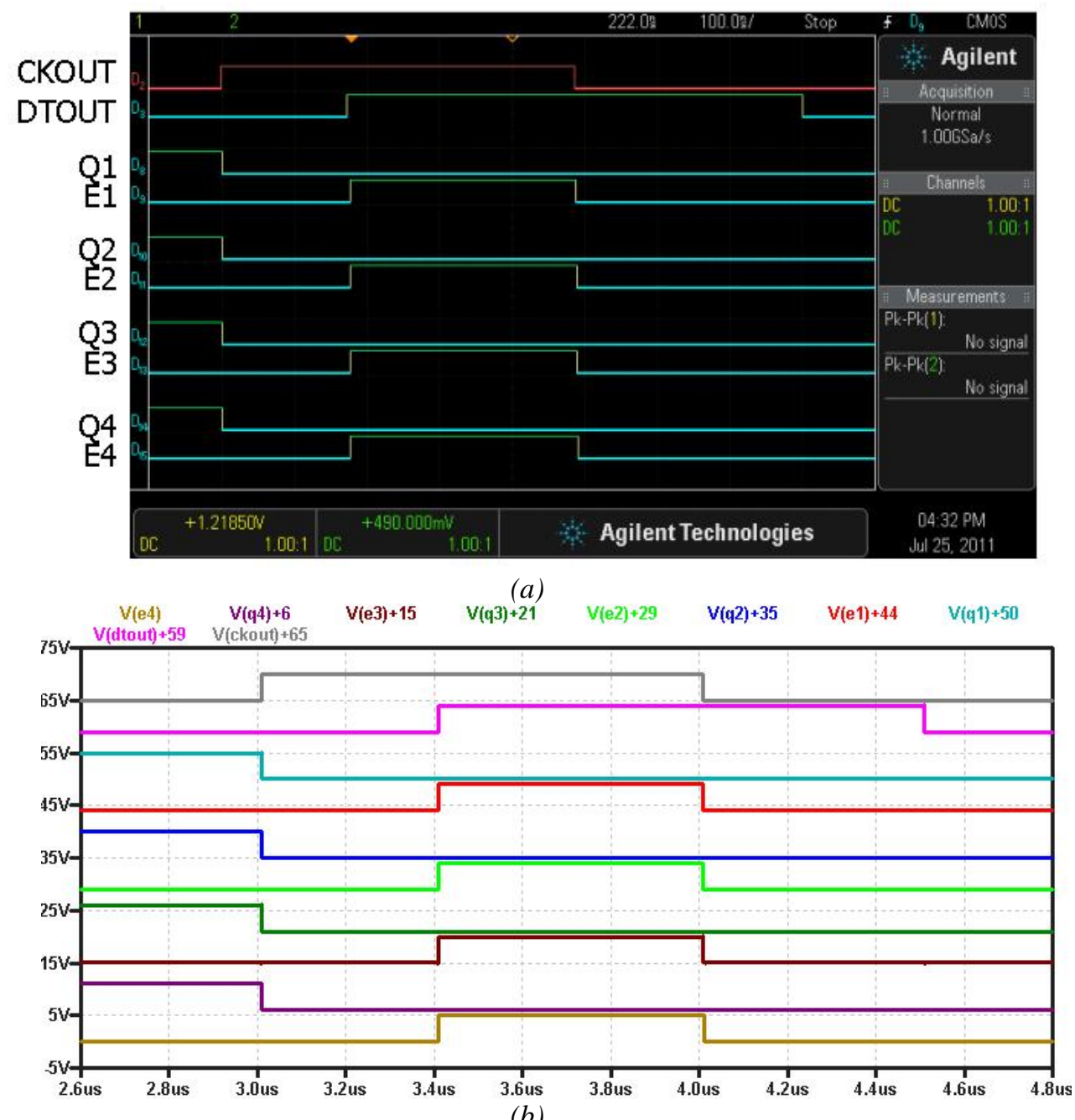

Fig. 4.7.3 Late Signal Test Waveforms (a) Chip Test Waveforms (b) SPICE Simulation Waveforms 


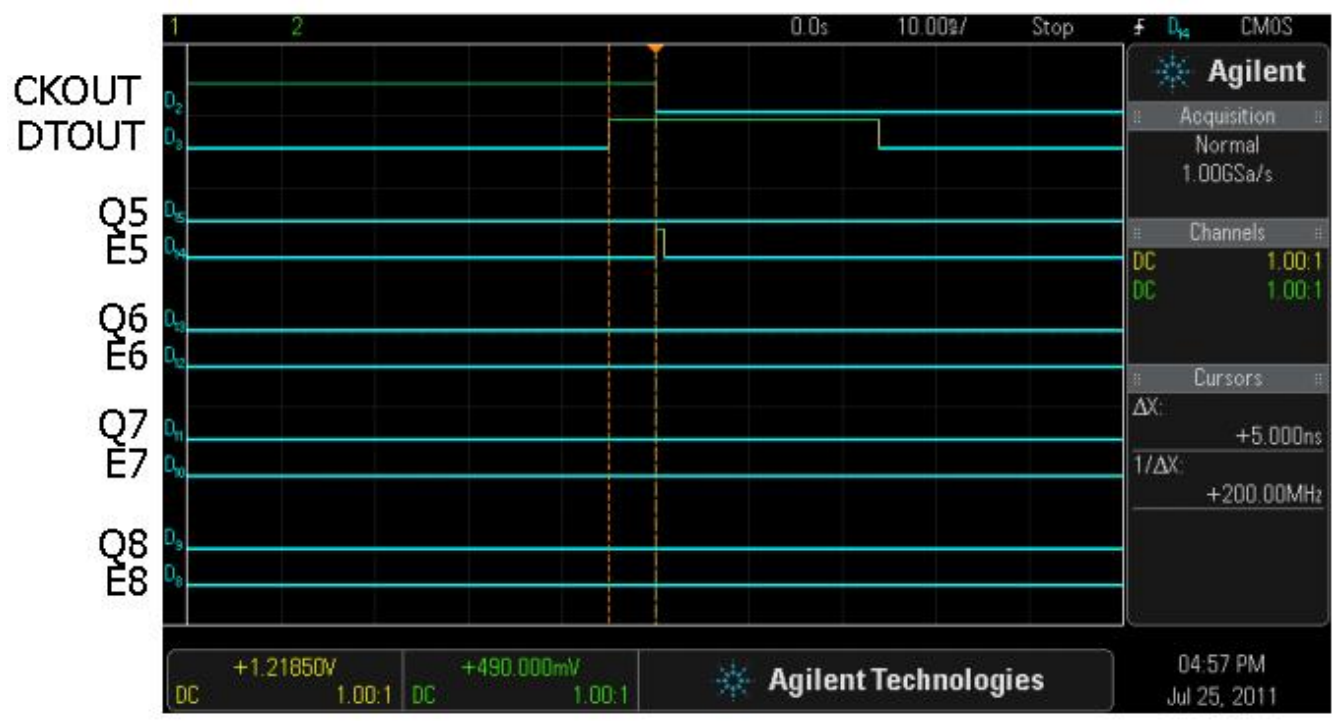

Fig. 4.7.4 Response Time Test

Future chip tests should focus on characterizing checker response times to various glitch conditions. These tests require precise error pattern generation beyond the capabilities of a regular function generator. The on-chip configurable delay chains are unable to deliver the precision required for robust testing. Further tests should utilize Automatic Test Pattern Generation (ATPG) methods to characterize the performance of each stability checker design.

Of 10 tested chips, only Chip 7 displayed a faulty checker. Fig. 4.7.5 shows SD6 of Chip 7 producing erroneous $\mathrm{Q}$ and $\mathrm{E}$ signals. The error was caused by a disconnected bonding wire on the SD6 checker output pads. The disconnected wire resulted from accidental physical contact during visual inspection. This could be prevented by covering the exposed die cavity with clear insulative tape. A photograph of the damage is shown in Fig. 4.7.6. 


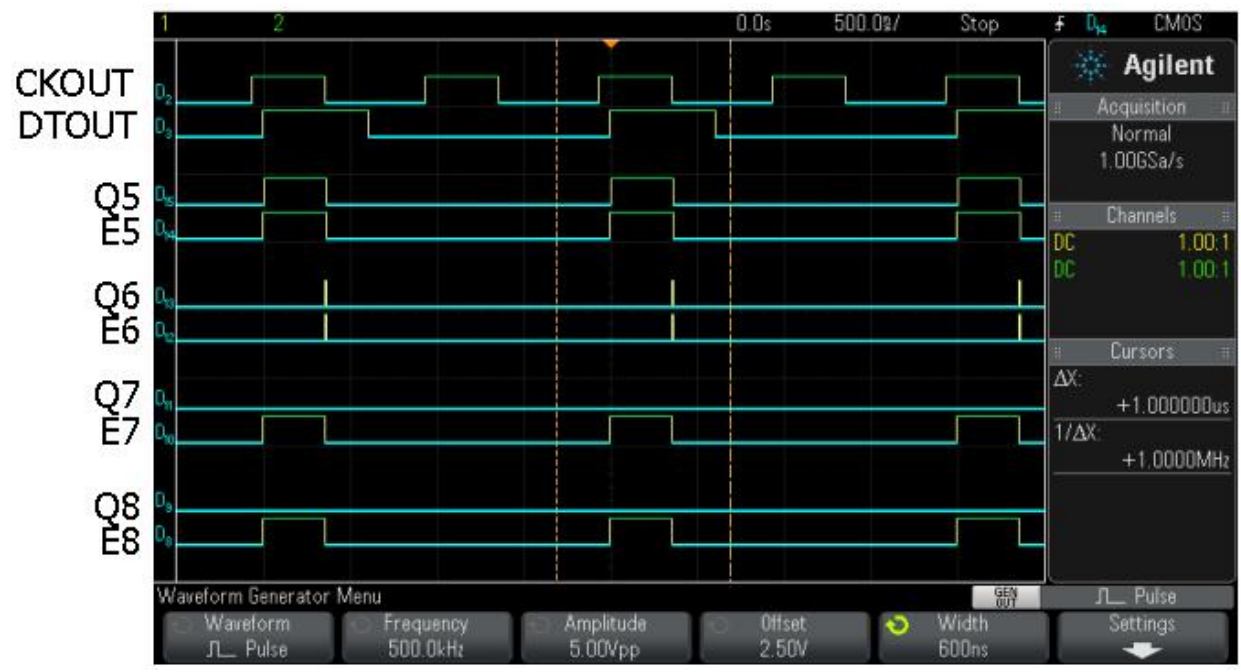

Fig. 4.7.5 Faulty Checker SD6 on Chip 7

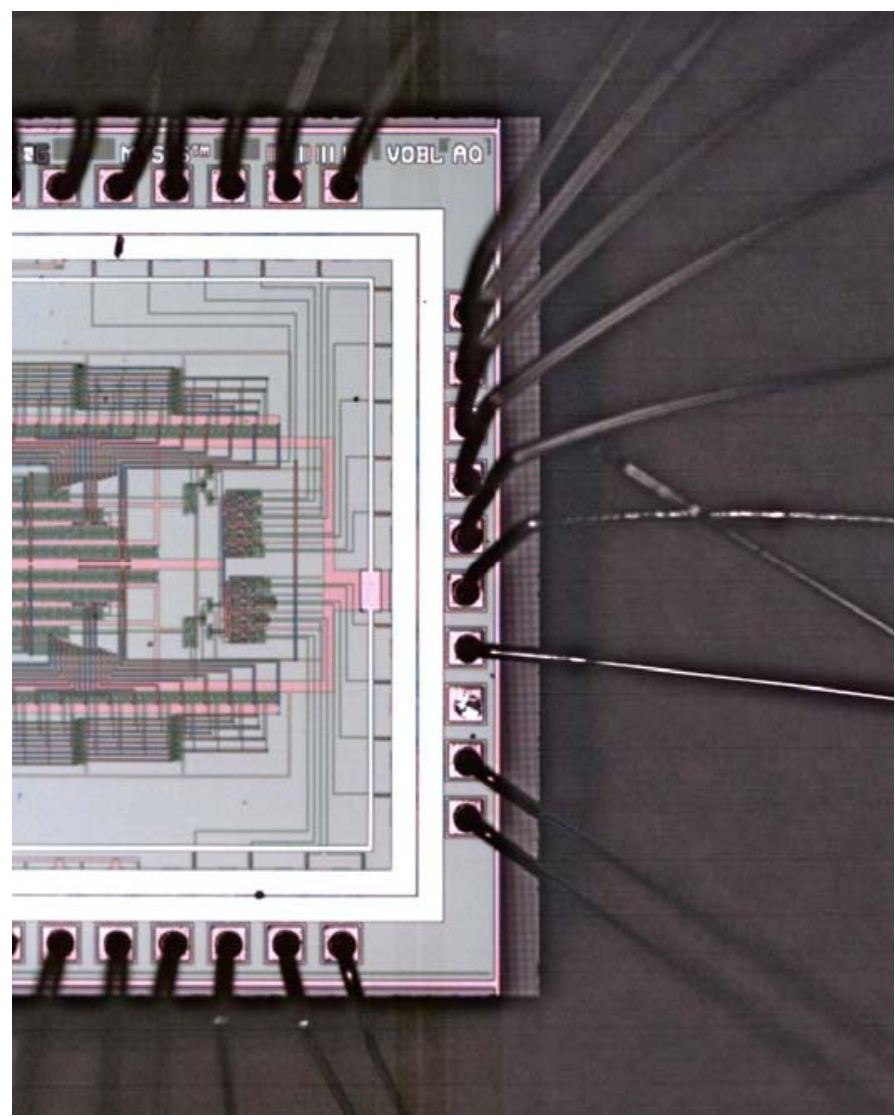

Fig. 4.7.6 Disconnected Wire on Chip 7 


\section{CHAPTER 5}

\section{Design Template}

\subsection{Design Tools}

The chip design project implemented for this study makes a viable template for potential student projects in the EE 431 course. This study has verified the tool chain for chip fabrication via the MEP and has also produced a base library of CMOS logic components for more complex designs.

The design process for this project was done entirely using the Electric VLSI Design tool [12]. It is an open-source Electronic Design Automation (EDA) tool originally written in $\mathrm{C}$ and later translated to Java. Electric supports many levels of circuit design, among which are CMOS, bipolar, schematics, and hardware description languages (HDL). It includes all essential tools for custom IC design including Design Rule Checking (DRC), Electrical Rule Checking (ERC), Network Consistency Checking/Layout versus Schematic (LVS) and simulation interfaces.

Most CAD tools utilize a connectivity approach in schematic design and a separate geometry approach in layout design. That is, the connectivity of components in schematics, which contains node connection information, is not linked to the geometric shapes designed in layout. As a result, conventional CAD tools require an additional node extraction process after a circuit layout has been drawn, in order for it to be verified against the schematics in LVS. This separation provides more flexibility in layout design. 
In contrast, Electric incorporates connectivity information in all phases of design, including layout. One of the main advantages of connectivity-based IC layout [33] is the simplification of the design iteration process. Typically, a layout has to be DRC clean before running it through LVS since the extractor cannot run if the design rules are wrong. If LVS errors are found, the layout has to be corrected and made DRC clean again. In Electric, with layout containing connectivity information, the designer needs only to verify LVS first and can then fix DRC errors without having to risk losing an LVS match. However, this scheme limits layout design flexibility.

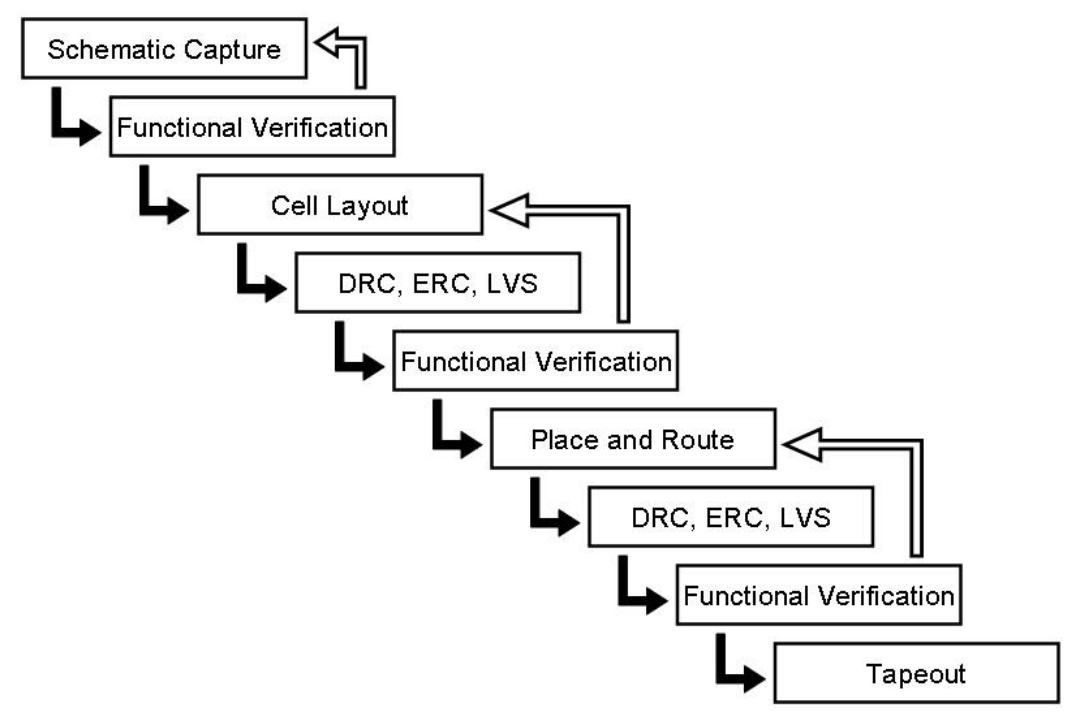

Fig. 5.1.1 Full Custom Design Flow

Electric is available in both binary and source versions. It comes pre-configured with MOSIS scalable CMOS design rules and requires minimal setup to configure it for the ON Semiconductor $\mathrm{C} 5$ process. Configuration instructions are provided in great detail by Dr. Jacob Baker in the CMOSedu website [34]. In addition to installation and setup instructions, CMOSedu also contains a collection of video tutorials that provide instruction on designing in Electric. 
As mentioned previously, Electric was configured to invoke LTSPICE for both schematic and layout simulations. The full custom design flow adopted for this project is illustrated in Fig. 5.1.1. Using a cell-based, bottom-up approach, each level of abstraction is verified for functionality in LTSPICE simulation and with layout checking tools.

\subsection{Template Project}

All cell layouts in the project library were drawn based on a set of custom-defined rules (Fig. 5.2.1). Cell heights were set at $100 \lambda$, measured from the outer edge of the power rail (N-well) to the outer edge of the ground rail (P-active). Power rails were $12 \lambda$ wide and are separated to the closest transistor by $8 \lambda$. N-well regions (pull-up) are separated by P-well regions (pull-down) by $22 \lambda$ to provide room for routing paths between abutting cells.

The template contains a 40-pin pad ring cell along with the individual pad ring components imported from the Electric library provided by CMOSedu. EE 431 students would be able to easily create custom systems and place them within the pad ring for full chip routing and simulation. Documentation on the pad ring architecture and subcomponents are available at [34]. Fig. 5.2.2 illustrates the pad ring template with a single standard cell inverter within it for a clearer visualization on the relative available chip area. 


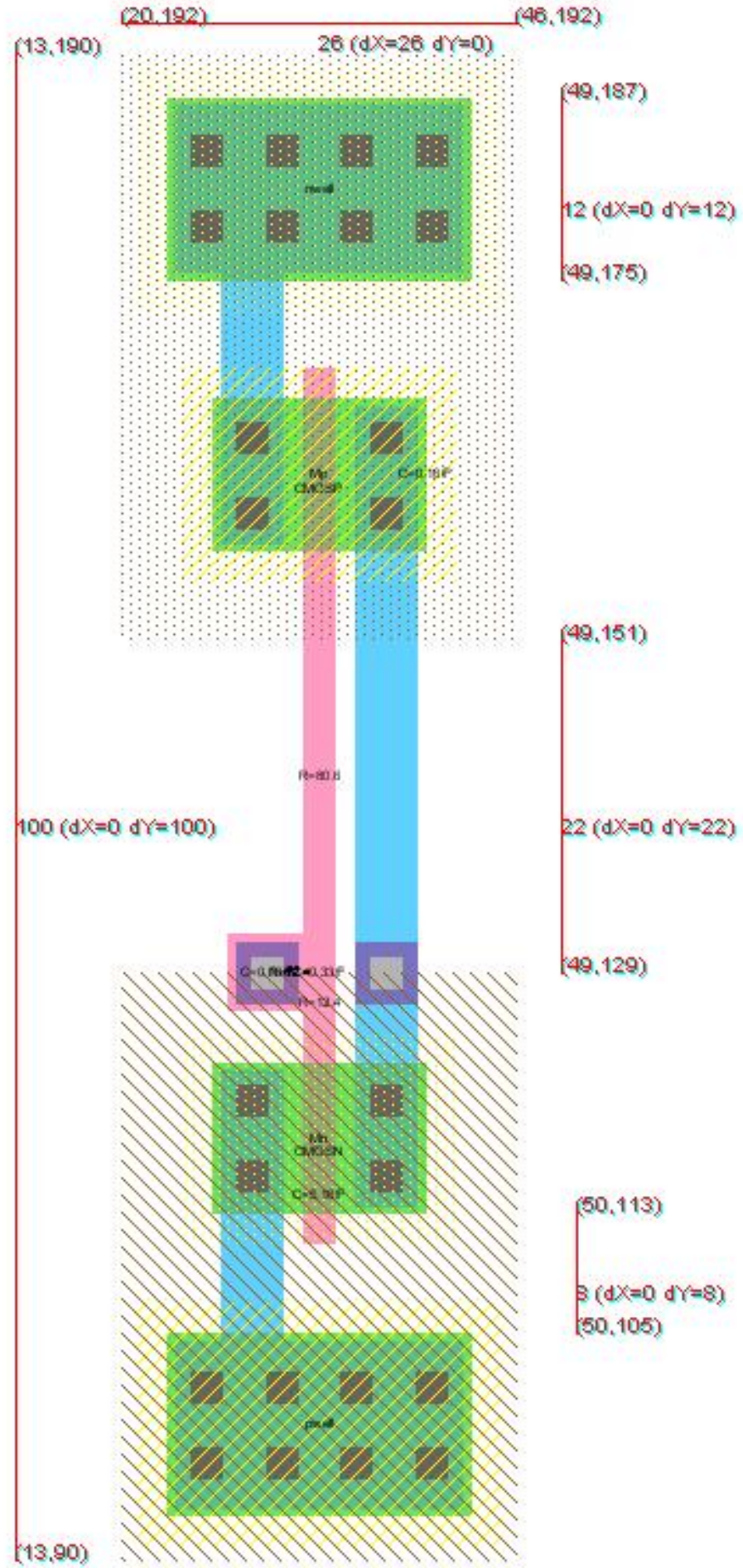

Fig. 5.2.1 Standard Cell Dimensions 


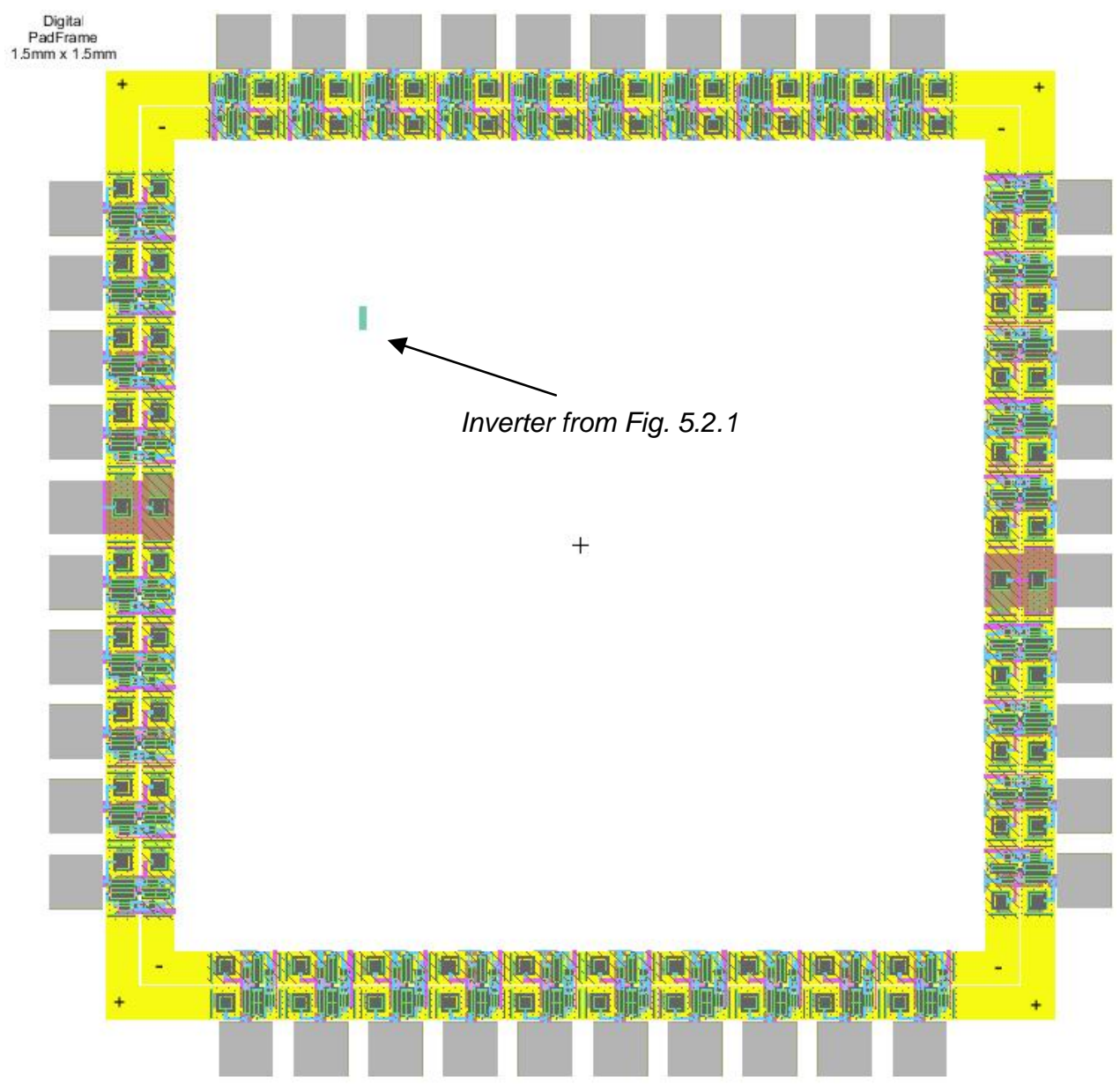

Fig. 5.2.2 Pad Ring Template 


\section{CHAPTER 6}

\section{Conclusions}

This thesis describes the design, manufacturing and testing process of a prototype chip containing stability checkers as part of the MOSIS Research Program, under MEP. The prototype chip consists of several stability checker modules, a configurable delay chain as well as routing logic to provide a testable platform for future device characterization.

Of 40 dice manufactured in the single lot, 10 were packaged in ceramic DIP 40 packages. Preliminary verification on the finished chips successfully demonstrated basic functionality. Actual delay chain measurements deviated estimates by approximately $3 \mathrm{x}$ for resolution and $4 \mathrm{x}$ for delay range. ATPG methods are recommended for further tests and characterization. The reader is referred to [40] on SPICE characterizations of stability checker designs.

Several delays occurred during the manufacturing process. The completed parts, which were initially projected to be completed on March 30, 2011, were returned on July 7, 2011 after two manufacturing issues at the wafer fabrication and chip packaging stages. Manufacturing delays should be taken in account if MEP is incorporated into the EE 431 course for class projects.

This project provides a usable design template for potential EE 431 student projects. The MOSIS service provides sufficient resources and tools to design and fabricate custom ICs. The Electric VLSI Design tool contains essential integrated tools 
for constructing and verifying the CMOS logic circuits used in this project. The SPICE interface included within the tool simplifies schematic and layout verification. The entire chip was designed from a blank template based on a full custom design flow.

Future projects based on this template should consider several issues encountered during chip design. First, the stability checkers were designed without regard for pad ring and chip package frequency response. Stability checker performance could be limited by chip ceramic DIP packaging. Simulation and testing should be carried out on the preexisting parts to determine optimal and maximum pad ring and package signal frequencies.

Unlike the stability checker design described in [10], the current system does not include output latches to capture error signals. The error signals from a set of variable sized stability checkers monitoring a common CUT output can be used to determine the magnitude of the detected delay fault. This improved design requires more rigorous simulation to determine optimal error transistor sizing given frequency and timing constraints.

Integrated testing logic consumes chip area in addition to the targeted CUT. In this case, the one-cold decoder consumes approximately 9x the area compared to the stability checker array. Most of this area was used for signal routing and can be minimized by compacting the cells at the cost of greater routing complexity and probability of design rule errors.

The lessons learned in this project are useful for future MEP projects. The author hopes that findings contributed with this thesis are useful for custom chip fabrication in future Cal Poly projects. 


\section{BIBLIOGRAPHY}

[1] White, M.; Due Vu; Due Nguyen; Ruiz, R.; Yuan Chen; Bernstein, J.B.; , "Product Reliability Trends, Derating Considerations and Failure Mechanisms with Scaled CMOS," Integrated Reliability Workshop Final Report, 2006 IEEE International , pp. 156-159, Oct. 16 2006-Sept. 192006

[2] Srinivasan, J.; Adve, S.V.; Bose, P.; Rivers, J.A.; , "The impact of technology scaling on lifetime reliability," Dependable Systems and Networks, 2004 International Conference on , pp. 177- 186, 28 June-1 July 2004

[3] Black, J.R.; , "Electromigration-A brief survey and some recent results," Electron Devices, IEEE Transactions on, vol. 16, no. 4, pp. 338- 347, Apr 1969

[4] Pedram, M.; Nazarian, S.; , "Thermal Modeling, Analysis, and Management in VLSI Circuits: Principles and Methods," Proceedings of the IEEE , vol. 94, no. 8, pp. 14871501, Aug. 2006

[5] Haggag, A.; Hess, K.; McMahou, W.; Register, L.F.; , "Impact of scaling on CMOS IC failure rate and design rules for reliability," Computational Electronics, 2000. Book of Abstracts. IWCE Glasgow 2000. 7th International Workshop on , pp. 49-50, 2000

[6] Simon Tam; Ping-Keung Ko; Chenming Hu; , "Lucky-electron model of channel hotelectron injection in MOSFET'S," Electron Devices, IEEE Transactions on , vol.31, no. 9, pp. 1116- 1125, Sep 1984 
[7] Rajesh, M.G.; Vinod, G.; Das, D.; Bhatnagar, P.V.; Pithawa, C.K.; Thaduri, A.; Verma, A.K.; , "A study of failure mechanisms in CMOS \& BJT ICs and their effect on device reliability," Reliability, Safety and Hazard (ICRESH), 2010 2nd International Conference on , pp. 425-430, 14-16 Dec. 2010

[8] Stankovic, T.R.; Stojcev, M.K.; Djordjevic, G.L.; , "Design of self-checking combinational circuits," Telecommunications in Modern Satellite, Cable and Broadcasting Service, 2003. TELSIKS 2003. 6th International Conference on , vol. 2, pp. $763-768$ vol. 2 , 1-3 Oct. 2003

[9] Lala, P.K.; Busaba, F.; Yarlagadda, K.C.; , "An approach for designing self-checking logic using residue codes ," VLSI Test Symposium, 1991. 'Chip-to-System Test Concerns for the 90's', Digest of Papers , pp. 166-171, 15-17 Apr 1991

[10] Franco, P.; McCluskey, E.J.; , "On-line delay testing of digital circuits," VLSI Test Symposium, 1994. Proceedings., 12th IEEE , pp. 167-173, 25-28 Apr 1994

[11] "MOSIS - Integrated Circuit Fabrication Service." Internet: http://www.mosis.com/, July 12, 2011 [July 14, 2011].

[12] "Static Free Software - Download." Internet: http://www.staticfreesoft.com/productsFree.html, July 12, 2011 [July 14, 2011].

[13] "Linear Technology - Design Simulation and Device Models." Internet: http://www.linear.com/designtools/software/, July 12, 2011 [July 14, 2011].

[14] "Europractice Homepage." Internet: http://www.europractice-ic.com/, July 12, 2011 [July 14, 2011]. 
[15] "CMP: Circuit Multi-Projets." Internet: http://cmp.imag.fr/, July 12, 2011 [July 14, 2011].

[16] "R. Jacob (Jake) Baker’s homepage." Internet: http://cmosedu.com/jbaker/jbaker.htm, June 26, 2011 [July 14, 2011].

[17] "E158 CMOS VLSI Design Spring 2007 MIPS Project." Internet: http://www4.hmc.edu:8001/Engineering/158/07/project/index.html, May 1, 2007 [July $14,2011]$.

[18] Franco, P.; McCluskey, E.J.; , "Delay Testing of Digital Circuits by Output Waveform Analysis," Test Conference, 1991, Proceedings., International , pp. 798, 26-30 Oct 1991

[19] Al-Asaad, H; Hayes, John P.; , "Design of Scalable Hardware Test Generators for Online BIST,” Digest of Papers: IEEE International On-Line Testing Workshop , pp. $164-167,1996$

[20] Fujiwara, H.; Yamamoto, A.; , "Parity-scan design to reduce the cost of test application," Computer-Aided Design of Integrated Circuits and Systems, IEEE Transactions on , vol. 12, no. 10, pp. 1604-1611, Oct 1993

[21] Saposhnikov, Vl.V.; Dmitriev, A.; Goessel, M.; Saposhnikov, V.V.; , "Self-dual parity checking-A new method for on-line testing," VLSI Test Symposium, 1996., Proceedings of 14th, pp. 162-168, 28 Apr-1 May 1996 
[22] Jha, N.K.; Wang, S.-J.; , "Design and synthesis of self-checking VLSI circuits and systems," Computer Design: VLSI in Computers and Processors, 1991. ICCD '91. Proceedings, 1991 IEEE International Conference on, pp. 578-581, 14-16 Oct 1991

[23] Favalli, M.; Metra, C.; , "Sensing circuit for on-line detection of delay faults," Very Large Scale Integration (VLSI) Systems, IEEE Transactions on , vol. 4, no. 1, pp. 130133, March 1996

[24] Metra, C.; Favalli, M.; Ricco, B.; , "On-line detection of logic errors due to crosstalk, delay, and transient faults," Test Conference, 1998. Proceedings., International , pp. 524-533, 18-23 Oct 1998

[25] Satish Yada; Bharadwaj Amrutur; Rubin A. Parekhji; , "Modified Stability Checking for On-line Error Detection," VLSI Design, 2007. Held jointly with 6th International Conference on Embedded Systems., 20th International Conference on , pp. 787-792, Jan. 2007

[26] Maymandi-Nejad, M. and Sachdev, M. "A digitally programmable delay element: design and analysis" Very Large Scale Integration (VLSI) Systems, IEEE Transactions on, vol. 11, no. 5, pp. 871-878, Oct. 2003.

[27] Maymandi-Nejad, M.; Sachdev, M.; , "A monotonic digitally controlled delay element," Solid-State Circuits, IEEE Journal of , vol. 40, no. 11, pp. 2212- 2219, Nov. 2005

[28] Geannopoulos, G. and Dai, X., "An adaptive digital deskewing circuit for clock distribution networks," Solid-State Circuits Conference, 1998. Digest of Technical Papers. 1998 IEEE International, pp. 400-401, Feb 1998 
[29] Saint-Laurent, M. and Swaminathan, M., "A digitally adjustable resistor for path delay characterization in high-frequency microprocessors," Mixed-Signal Design, 2001. SSMSD. 2001 Southwest Symposium on, pp. 61-64, 2001

[30] "History of the MOSIS Educational Program." Internet: http://www.mosis.com/products/mep/mep-history.html, July 12, 2011 [July 14, 2011].

[31] "What Is the MOSIS Educational Program?." Internet: http://mosis.com/products/mep/mep-about.html, July 14, 2011 [July 14, 2011].

[32] "ON Semiconductor Rules and Technology Files." Internet: http://mosis.com/on_semi/on_semi_rules_libs.html, July 14, 2011 [July 14, 2011].

[33] "Static Free Software - New Concepts in Electric." Internet: http://www.staticfreesoft.com/electricDifferent.html, July 14, 2011 [July 14, 2011].

[34] "Electric at CMOSedu.com." Internet: http://cmosedu.com/cmos1/electric/electric.htm, March 2, 2011 [July 14, 2011].

[35] "Submit A Design To MOSIS Chart." Internet: http://mosis.com/support/submit/, July 14, 2011 [July 14, 2011].

[36] Weste, N.H.E. and Harris, D.M. CMOS VLSI Design: A Circuits and Systems Perspective. Boston, MA: Pearson/Addison-Wesley, 2005, pp. 405.

[37] “pkg-dip40-char.pdf” Internet: http://www.mosis.com/Technical/Packaging/Ceramic/pkg-dip40-char.pdf, June 1, 1998 [July 14, 2011]. 
[38] “SCMOS Rules" Internet:

http://www.mosis.com/Technical/Designrules/scmos/scmos-main.html\#tech-codes, July 14, 2011 [July 14, 2011].

[39] Masleid R.P. "Configurable delay chain with stacked inverter delay elements" U.S. 7592842, Sept. 22, 2009.

[40] Jacobvitz, A., "On-line Stability Checkers for Sequential Circuit Elements," Senior Project Report, California Polytechnic State University, San Luis Obispo, 2009.

[41] R. J. Baker. CMOS Circuits Design, Layout and Simulation.. Piscataway, NJ: IEEE Press, 2008, pp. 97. 


\section{Appendix A: Endorsement Letter for MOSIS Subsidy}

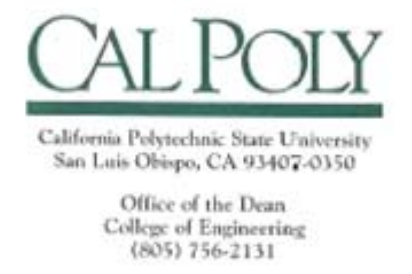

September 28,2010

The MOSIS Service

4676 Admiralty Way

Marina del Rey, CA 90292-6695

To Whom it May Concern:

This letter is in support of Dr. John Oliver's effort to build a custom chip using the services provided by MOSIS. Dr. Oliver does not have outside funding for his experimental chip.

The support MOSIS provides will enable our students to build custom chips as part of the EE 431 VLSI design course.

The services provided to Cal Poly by MOSIS are greatly appreciated. Through the generous support of organizations such yours, our students continually benefit from the hands-on "Learn By Doing" credo that sets Cal Poly engineering students apart from other institutions.

Again, thank you for your generosity.

Sincerely,

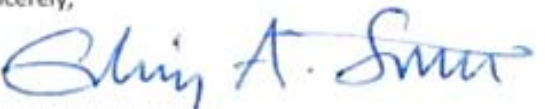

Erling Smith, Ph.D., P|E.

Acting Dean

College of Engineering 


\section{Appendix B: MOSIS Price Quotation}

MOSIS QUOTATION

To: John Oliver

California Polytechnic State University

Electrical Engineering Dept.

One Grand Ave

BIdg 20, Room 200

San Luis Obispo, CA 93407-0355

clee83@calpoly.edu

Issue Purchase Order to: University of Southern Califomia

Tax ID: $85-1642394$

Quote ID: 159375-A

Date: 16 -SEP-2010

Expires: 16-OCT-2010

Payment Terms: See below

MOSIS Account: 4186-MEP-RES/CALPOLY-EE

The packaging price in this quote reflects the discounted prices that MOSIS offers to (approved) MEP-RES projects; it is not applicable to any other projects.

These prices include Federal Express 2 day shipping; delivery is best effort.

Terms and conditions are defined in the customer agreement signed by your organization on 10-DEC-2004.

For more information please contact MOSIS customer support.

Automated Quote Generator

\section{CONFIDENTIAL}

The MOSIS Service

USC Information Sciences Institute 4676 Admiralty Way

Marina del Rey, CA 90292-6695

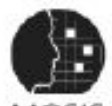

MOSIS
Telephone $(310) 448-9400$

Facsimile (310) 823-5624 support@mosis.com http://www.mosis.com 


\title{
Appendix C: MOSIS ON Semiconductor C5 Characterization Data and SPICE Models
}

\author{
MOSIS WAFER ELECTRICAL TESTS \\ RUN: VOBL \\ TECHNOLOGY: SCNO5 \\ VENDOR: AMIS (ON-SEMI) \\ Run type: SHR

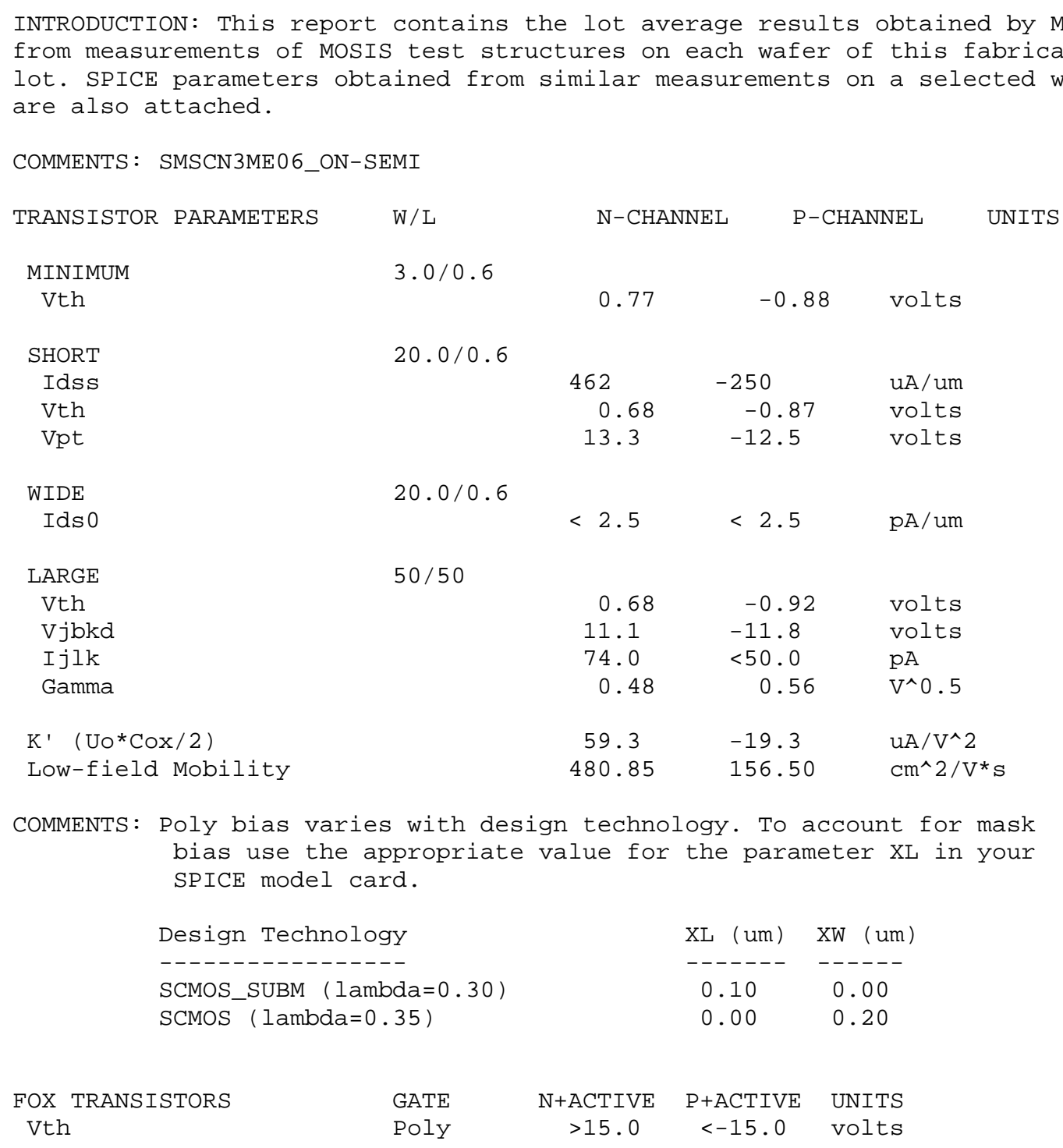

FEATURE SIZE: 0.5 microns 


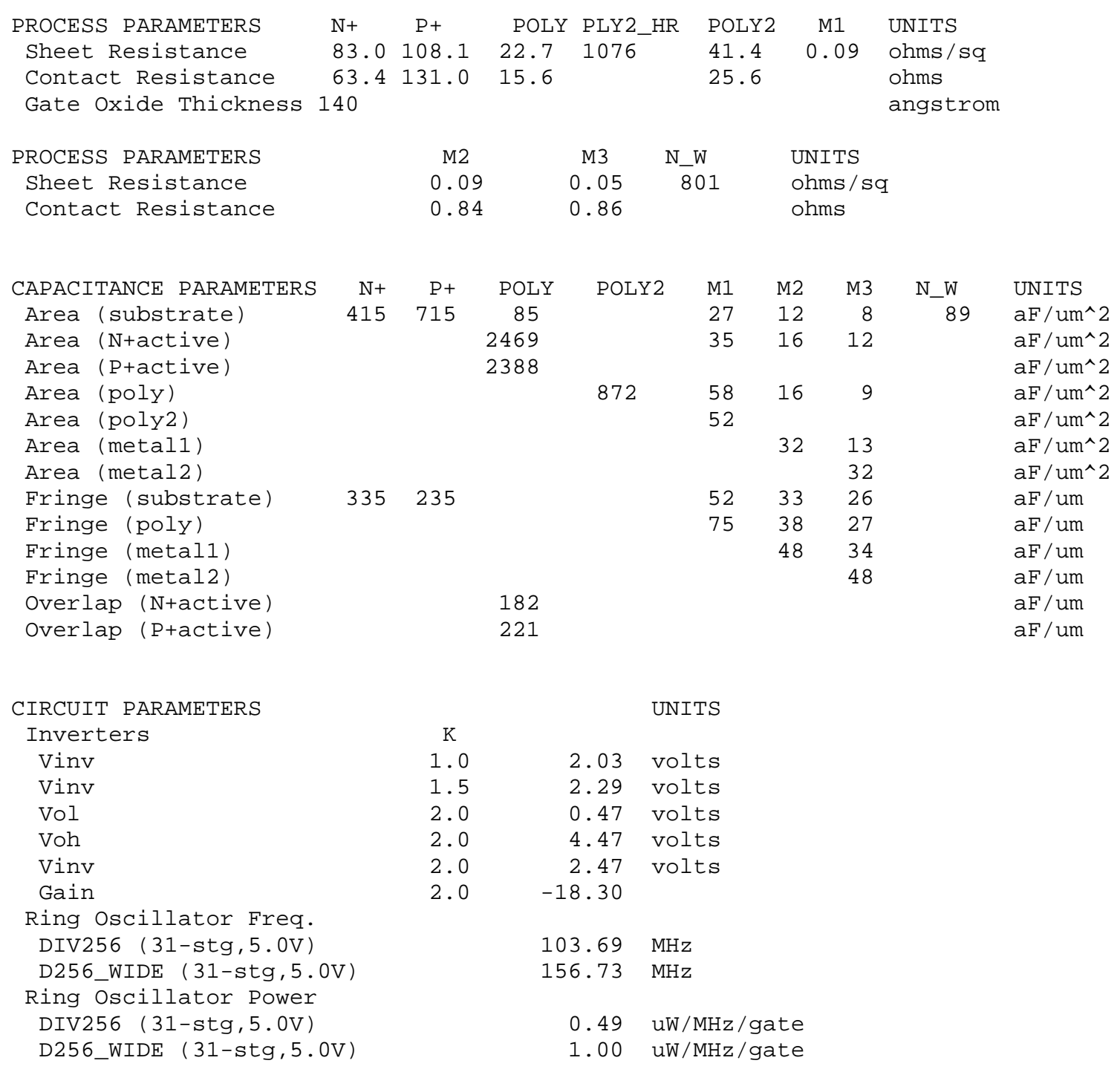

COMMENTS: SUBMICRON 
VOBL SPICE BSIM3 VERSION 3.1 PARAMETERS

SPICE $3 f 5$ Level 8, Star-HSPICE Level 49, UTMOST Level 8

* DATE: Jun $28 / 11$

* LOT: VOBL WAF : 8101

* Temperature_parameters=Default . MODEL CMOSN NMOS (

$\begin{array}{ll}+\mathrm{VERSION} & =3.1 \\ +\mathrm{XJ} & =1.5 \mathrm{E}-7\end{array}$

$+\mathrm{K} 1=0.9066799$

$+\mathrm{K} 3 \mathrm{~B}=-9.0554284$

+ DVTOW $=0$

+ DVTO $=0.7245866$

$+\mathrm{U} \odot=455.9385207$

$+\mathrm{UC}=8.217834 \mathrm{E}-12$

+ AGS $=0.1328908$

+ KETA $=-4.157626 \mathrm{E}-3$

$+\mathrm{RDSW}=1.124424 \mathrm{E} 3$

$+W R=1$

$+\mathrm{XL}=1 \mathrm{E}-7$

+ DWB $\quad=2.52186 \mathrm{E}-8$

+ CIT $=0$

$+\mathrm{CDSCB}=0$

+ DSUB $=0.0506449$

+ PDIBLC2 $=1.190431 \mathrm{E}-3$

+ PSCBE1 $=1.659137 \mathrm{E} 10$

+ DELTA $=0.01$

+ PRT $=0$

$+\mathrm{KT} 1 \mathrm{~L}=0$

$+\mathrm{UB1}=-7.61 \mathrm{E}-18$

$+W L=0$

$+W W N \quad=1$

+ LLN $\quad=1$

+ LWL $\quad=0$

+ CGDO $=1.82 \mathrm{E}-10$

$+\mathrm{CJ} \quad=4.143407 \mathrm{E}-4$

$+\mathrm{CJSW}=3.377962 \mathrm{E}-10$

+ CJSWG $=1.64 \mathrm{E}-10$

$+\mathrm{CF}=0$

+ PK2 $=-0.070569$

*

\begin{tabular}{|c|c|}
\hline TNOM & $=27$ \\
\hline $\mathrm{NCH}$ & $=1.7 \mathrm{E} 17$ \\
\hline K2 & $=-0.1049093$ \\
\hline W० & $=4.37907 \mathrm{E}-8$ \\
\hline DVT1W & $=0$ \\
\hline DVT1 & $=0.3384454$ \\
\hline UA & $=1.028307 \mathrm{E}-13$ \\
\hline VSAT & $=1.994269 \mathrm{E} 5$ \\
\hline B० & $=1.94105 \mathrm{E}-6$ \\
\hline A1 & $=1.63163 \mathrm{E}-4$ \\
\hline PRWG & $=0.1016734$ \\
\hline WINT & $=2.018804 \mathrm{E}-7$ \\
\hline XW & $=0$ \\
\hline VOFF & $=-6.4984 \mathrm{E}-5$ \\
\hline CDSC & $=2.4 \mathrm{E}-4$ \\
\hline ETA○ & $=3.293149 \mathrm{E}-3$ \\
\hline PCLM & $=2.0160615$ \\
\hline PDIBLCB & $=-0.26839$ \\
\hline PSCBE2 & $=3.299403 \mathrm{E}-9$ \\
\hline RSH & $=83$ \\
\hline UTE & $=-1.5$ \\
\hline KT2 & $=0.022$ \\
\hline UC1 & $=-5.6 \mathrm{E}-11$ \\
\hline WLN & $=1$ \\
\hline WWL & $=0$ \\
\hline LW & $=0$ \\
\hline CAPMOD & $=2$ \\
\hline CGSO & $=1.82 \mathrm{E}-10$ \\
\hline PB & $=0.8385926$ \\
\hline PBSW & $=0.8$ \\
\hline PBSWG & $=0.8$ \\
\hline PVTHO & $=-0.0411169$ \\
\hline WKETA & $=-0.0103973$ \\
\hline
\end{tabular}

\begin{tabular}{|c|c|}
\hline LEVEL & $=49$ \\
\hline TOX & $=1.4 \mathrm{E}-8$ \\
\hline VTHO & $=0.6157347$ \\
\hline K3 & $=20.5641789$ \\
\hline NLX & $=1.547679 \mathrm{E}-9$ \\
\hline DVT2W & $=0$ \\
\hline DVT2 & $=-0.5$ \\
\hline UB & $=1.441158 \mathrm{E}-18$ \\
\hline$A \odot$ & $=0.5692029$ \\
\hline B1 & $=5 \mathrm{E}-6$ \\
\hline $\mathrm{A} 2$ & $=0.3$ \\
\hline PRWB & $=7.487028 \mathrm{E}-3$ \\
\hline LINT & $=8.000982 \mathrm{E}-8$ \\
\hline DWG & $=-3.515558 \mathrm{E}-9$ \\
\hline NFACTOR & $=1.0533122$ \\
\hline CDSCD & $=0$ \\
\hline ETAB & $=-1.445104 \mathrm{E}-3$ \\
\hline PDIBLC1 & $=4.138667 \mathrm{E}-4$ \\
\hline DROUT & $=1.600548 \mathrm{E}-4$ \\
\hline PVAG & $=0$ \\
\hline MOBMOD & $=1$ \\
\hline KT1 & $=-0.11$ \\
\hline UA1 & $=4.31 E-9$ \\
\hline AT & $=3.3 E 4$ \\
\hline WW & $=0$ \\
\hline LL & $=0$ \\
\hline LWN & $=1$ \\
\hline XPART & $=0.5$ \\
\hline CGBO & $=1 \mathrm{E}-9$ \\
\hline MJ & $=0.4274223$ \\
\hline MJSW & $=0.2056208$ \\
\hline MJSWG & $=0.2056208$ \\
\hline PRDSW & $=257.844173$ \\
\hline LKETA & $=-2.677742 \mathrm{E}-3$ \\
\hline
\end{tabular}




\begin{tabular}{|c|c|}
\hline $\begin{array}{l}\text {. MODEL CI } \\
+ \text { +VERSION }\end{array}$ & $\begin{array}{l}\text { MOSP PMOS } \\
=31\end{array}$ \\
\hline$+\mathrm{XJ}$ & $=1.5 \mathrm{E}-7$ \\
\hline$+\mathrm{K} 1$ & $=0.553472$ \\
\hline$+\mathrm{K} 3 \mathrm{~B}$ & $=1.9253903$ \\
\hline +DVTOW & $=0$ \\
\hline +DVTO & $=0.514863$ \\
\hline$+\mathrm{U} \odot$ & $=201.3603195$ \\
\hline$+U C$ & $=-1 \mathrm{E}-10$ \\
\hline$+\mathrm{AGS}$ & $=0.1149535$ \\
\hline +KETA & $=-4.865785 \mathrm{E}-3$ \\
\hline +RDSW & $=3 \mathrm{E} 3$ \\
\hline$+W R$ & $=1$ \\
\hline$+X L$ & $=1 \mathrm{E}-7$ \\
\hline$+\mathrm{DWB}$ & $=-1 \cdot 249313 \mathrm{E}-8$ \\
\hline+ CIT & $=0$ \\
\hline$+\mathrm{CDSCB}$ & $=0$ \\
\hline +DSUB & $=0.463836$ \\
\hline +PDIBLC2 & $=3.119375 \mathrm{E}-3$ \\
\hline +PSCBE1 & $=1 \mathrm{E} 8$ \\
\hline+ DELTA & $=0.01$ \\
\hline +PRT & $=0$ \\
\hline +KT1L & $=0$ \\
\hline +UB1 & $=-7.61 \mathrm{E}-18$ \\
\hline$+W L$ & $=0$ \\
\hline$+W W N$ & $=1$ \\
\hline +LLN & $=1$ \\
\hline$+L W L$ & $=0$ \\
\hline$+\mathrm{CGDO}$ & $=2.21 \mathrm{E}-10$ \\
\hline$+\mathrm{CJ}$ & $=7.158953 \mathrm{E}-4$ \\
\hline +CJSW & $=2.387118 \mathrm{E}-10$ \\
\hline$+C J S W G$ & $=6.4 \mathrm{E}-11$ \\
\hline$+\mathrm{CF}$ & $=0$ \\
\hline +PK2 & $=3.73981 \mathrm{E}-3$ \\
\hline
\end{tabular}

\begin{tabular}{|c|c|}
\hline TNOM & $=27$ \\
\hline $\mathrm{NCH}$ & $=1.7 \mathrm{E} 17$ \\
\hline K2 & $=7.871921 \mathrm{E}-3$ \\
\hline W० & $=8.102064 \mathrm{E}-7$ \\
\hline DVT1W & $=0$ \\
\hline DVT1 & $=0.2591276$ \\
\hline UA & $=2.408572 \mathrm{E}-9$ \\
\hline VSAT & $=8.054026 \mathrm{E} 4$ \\
\hline $\mathrm{B} \odot$ & $=7.002197 \mathrm{E}-7$ \\
\hline A1 & $=3.43526 \mathrm{E}-4$ \\
\hline PRWG & $=-\odot .0260546$ \\
\hline WINT & $=2.205007 \mathrm{E}-7$ \\
\hline XW & $=0$ \\
\hline VOFF & $=-0.082897$ \\
\hline CDSC & $=2.4 \mathrm{E}-4$ \\
\hline ETAO & $=3.421086 \mathrm{E}-4$ \\
\hline PCLM & $=2.3991271$ \\
\hline PDIBLCB & $=-8.697031 \mathrm{E}-3$ \\
\hline PSCBE2 & $=3.386423 \mathrm{E}-9$ \\
\hline RSH & $=108.1$ \\
\hline UTE & $=-1.5$ \\
\hline KT2 & $=0.022$ \\
\hline UC1 & $=-5.6 \mathrm{E}-11$ \\
\hline WLN & $=1$ \\
\hline WWL & $=0$ \\
\hline LW & $=0$ \\
\hline CAPMOD & $=2$ \\
\hline CGSO & $=2.21 \mathrm{E}-10$ \\
\hline PB & $=0.8709343$ \\
\hline PBSW & $=0.8$ \\
\hline PBSWG & $=0.8$ \\
\hline PVTHO & $=5.98016 \mathrm{E}-3$ \\
\hline WKETA & $=0.0107068$ \\
\hline
\end{tabular}

\begin{tabular}{|c|c|}
\hline LEVEL & $=49$ \\
\hline TOX & $=1.4 \mathrm{E}-8$ \\
\hline VTHO & $=-0.9152268$ \\
\hline K3 & $=0$ \\
\hline NLX & $=6.679093 \mathrm{E}-9$ \\
\hline DVT2W & $=0$ \\
\hline DVT2 & $=-0.3$ \\
\hline UB & $=1 \mathrm{E}-21$ \\
\hline$A \odot$ & $=0.6910763$ \\
\hline B1 & $=0$ \\
\hline A2 & $=0.6978261$ \\
\hline PRWB & $=-0.051516$ \\
\hline LINT & $=1.277302 \mathrm{E}-7$ \\
\hline DWG & $=7.754013 \mathrm{E}-11$ \\
\hline NFACTOR & $=0.7608126$ \\
\hline CDSCD & $=0$ \\
\hline ETAB & $=-0.0129742$ \\
\hline PDIBLC1 & $=0.0364452$ \\
\hline DROUT & $=0.2269675$ \\
\hline PVAG & $=0.0150014$ \\
\hline MOBMOD & $=1$ \\
\hline KT1 & $=-0.11$ \\
\hline UA1 & $=4.31 \mathrm{E}-9$ \\
\hline AT & $=3.3 E 4$ \\
\hline WW & $=0$ \\
\hline LL & $=0$ \\
\hline LWN & $=1$ \\
\hline XPART & $=0.5$ \\
\hline CGBO & $=1 \mathrm{E}-9$ \\
\hline MJ & $=0.486754$ \\
\hline MJSW & $=0.2078274$ \\
\hline MJSWG & $=0.2079833$ \\
\hline PRDSW & $=14.8598424$ \\
\hline LKETA & $=-0.0138209$ \\
\hline
\end{tabular}




\section{Appendix D: Full Chip Routing Layers}

\section{Layer 1 - Diffusion and Metal 1}

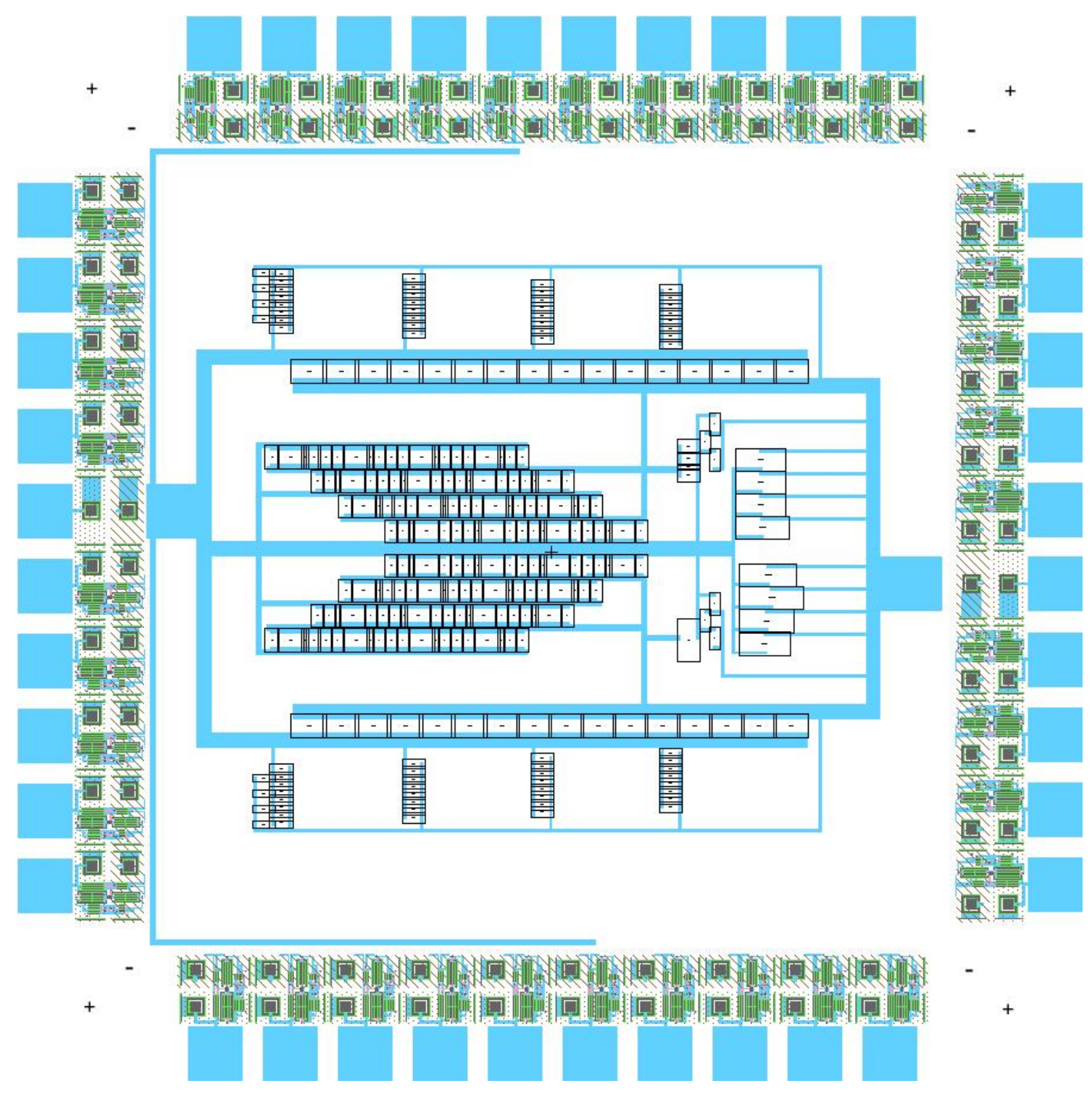




\section{Layer 2 - Layer 1 and Metal 2}

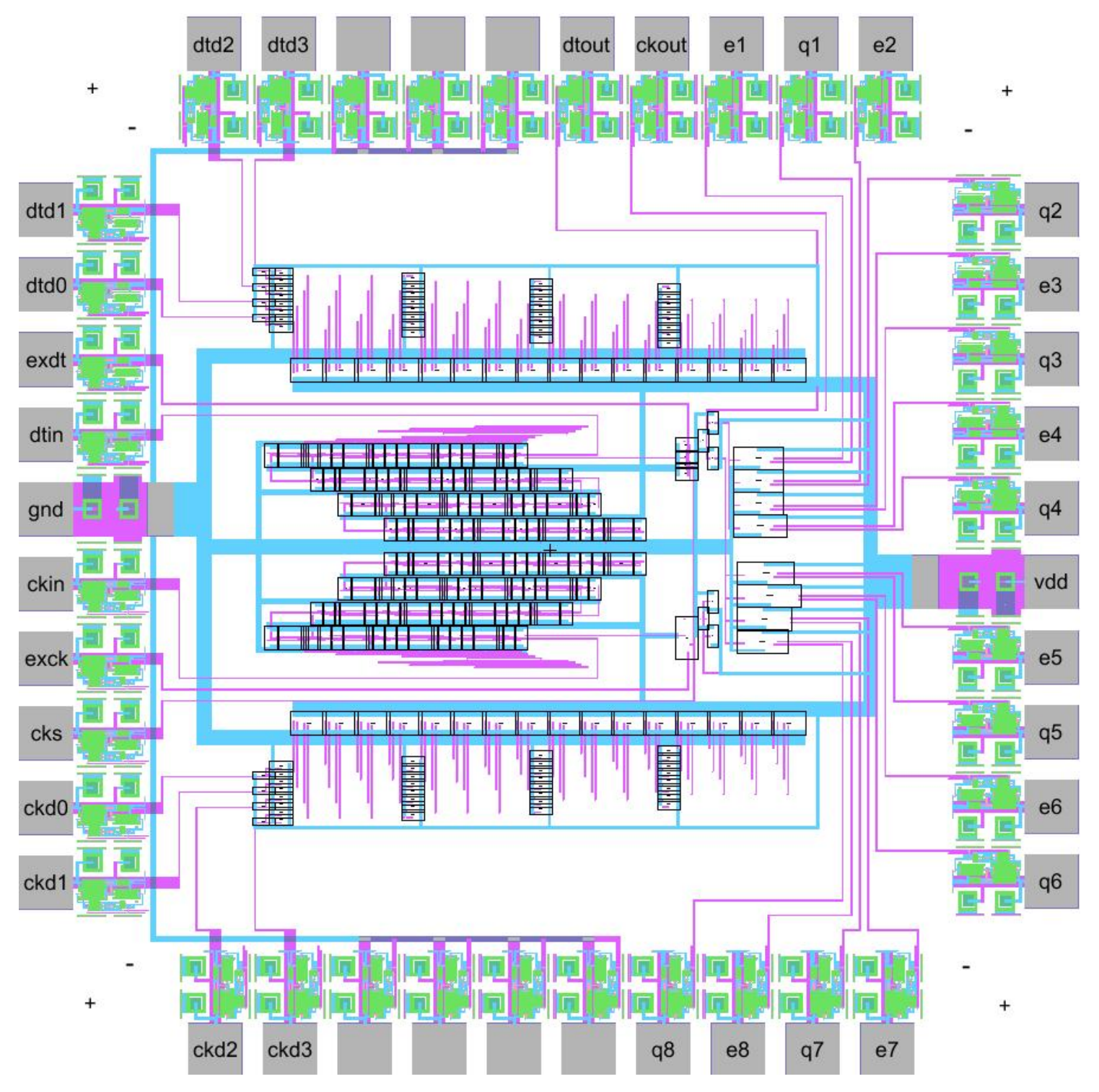




\section{Layer 3 - Layer 2 and Metal 3}

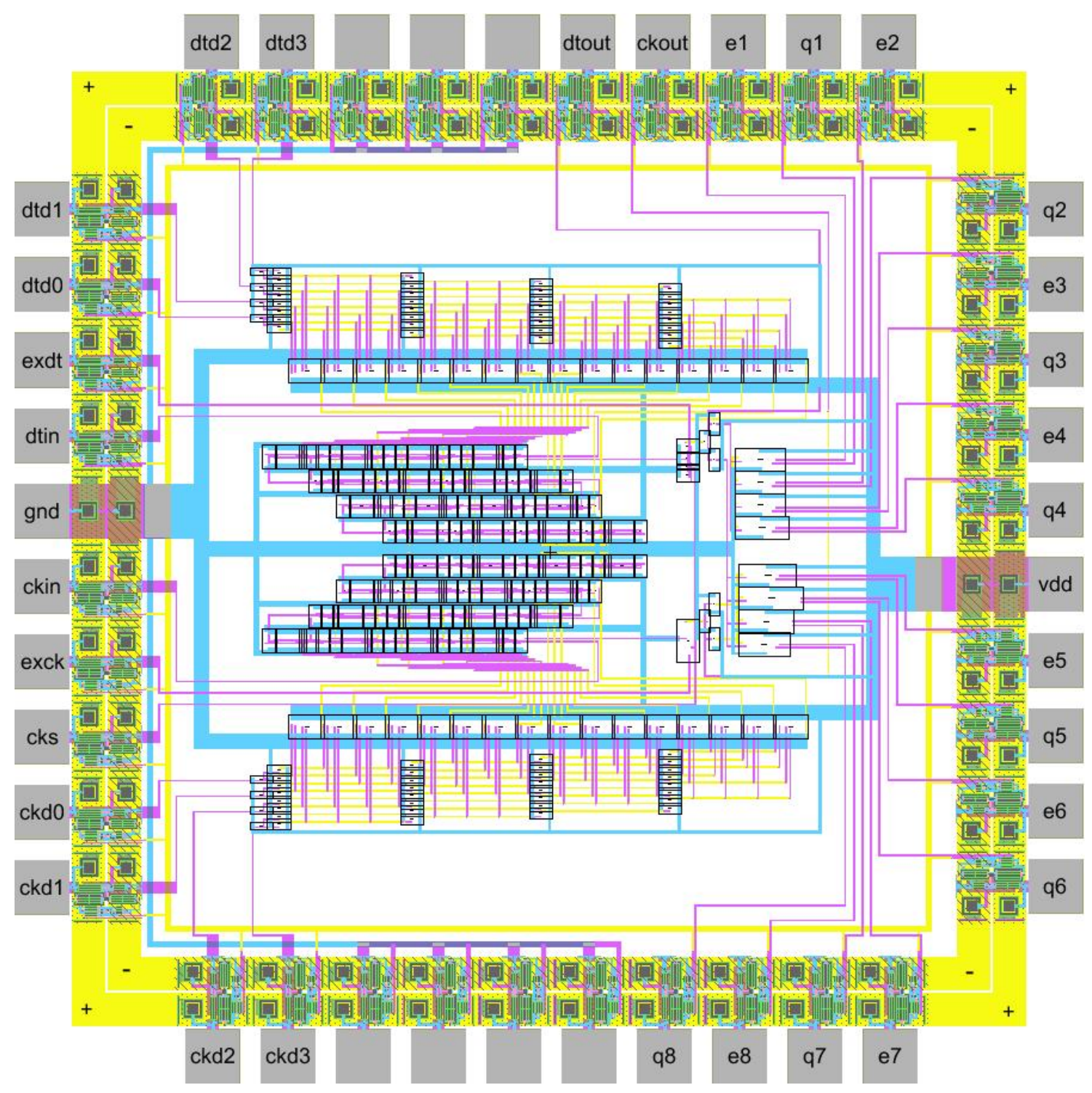




\section{Appendix E: SPICE Code for Post-Silicon Verification}

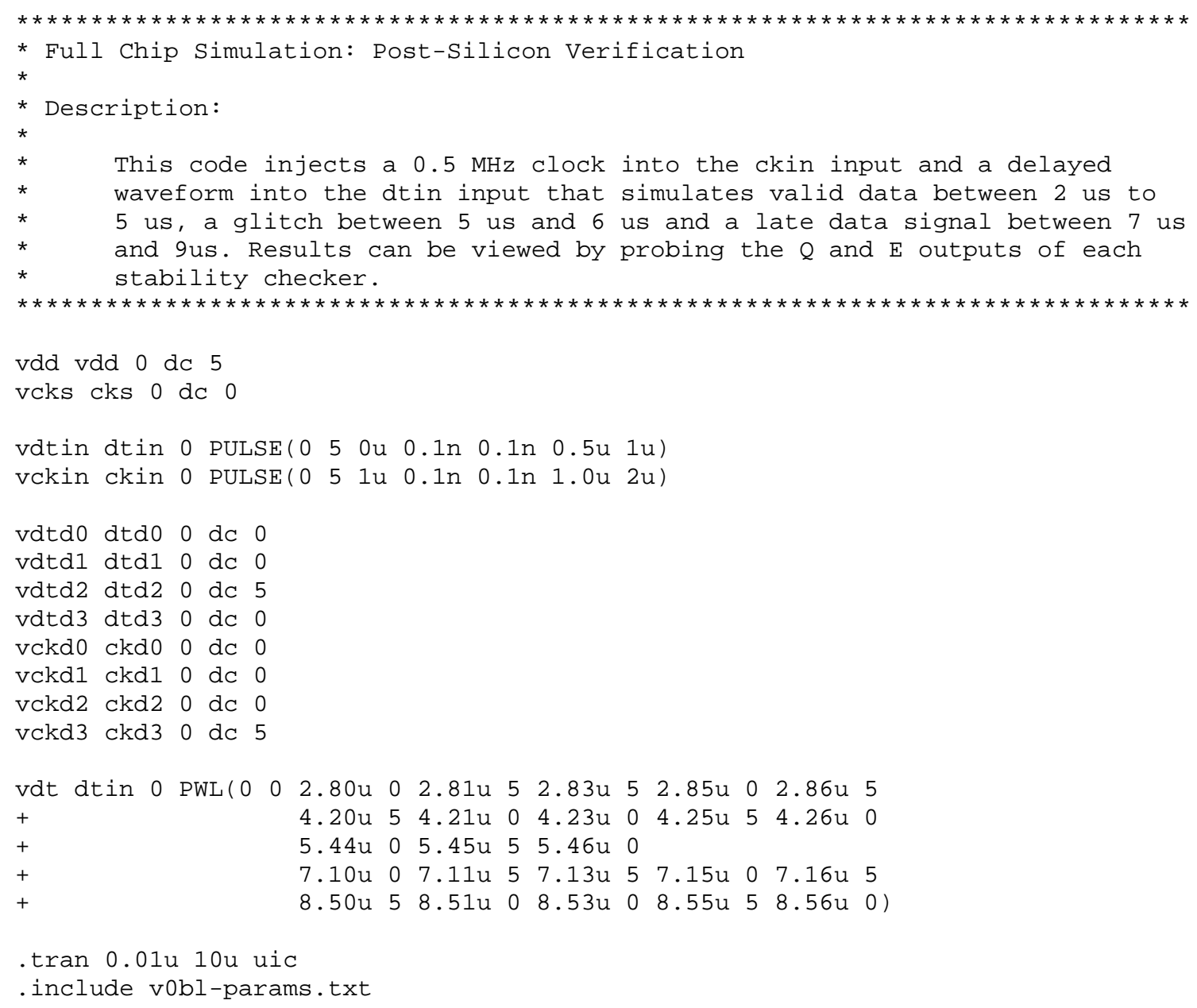




\title{
Appendix F: Manufacturability Review Results
}

\section{Funding authorization pending (Stability Detectors)}

\author{
The MOSIS Service <xmosis@mosis.com> \\ To: clee83@calpoly.edu \\ Manufacturability Review has completed and returned these results: \\ Project Warnings: \\ POLY layer drawn density is $1.3 \%$; minimum required by AMI_CSF \\ is $12.0 \%$. \\ METAL1 layer drawn density is $26.1 \%$; minimum required by \\ AMI_C5F is $30.0 \%$. \\ METAL2 layer drawn density is $17.5 \%$; minimum required by \\ AMI_C5F is $30.0 \%$. \\ Due to the noted low layer densitites, this project may be \\ excluded from the next run. \\ Account 4186 has no current funding authorization. \\ Project Status:
}

Design 84258 status: FUNDING AUTHORIZATION PENDING

Design name: Stability Detectors

E-mail address: clee83@calpoly.edu

Phone number: (805) 455-0690

Technology: SCN3M_SUBM, lambda $=.3$

Fabrication restricted to AMI only.

Fill to be added: No

This project can be fabricated on a AMI_CSF run using C5N or C5N_BULK or C5F or C5T.

Run date requested: $27-$ SEP-2010

Layout format: GDS

Top or root structure is "fullchip".

Layout file: complete; Binary CRC checksum: 2260758084, 2258944

Intended disposition: RESEARCH

Bonding pads: 40

Layout size: $1490 \times 1490$ microns; area: 2.217 sq millimeters

Layers found (and densities): CONTACT, P_WELL, N_WELL, ACTIVE, P_PLUS_SELECT, N_PLUS_SELECT, PŌLY $(1.3 \%)$, METAL1 $(26.1 \%)$, VIA, METAL2 (17.5\%), GLASS, VIA2, METAL3

Requested quantity: 40

Requested packaging: DIP40 [MOSIS generated bonding diagram]

(10 parts), UNPACKAGED (30 parts)

Maximum die size: $2250 \times 2250$

A total of 40 parts are ordered

with 10 to be packaged in DIP40

with 30 to be delivered unpackaged 


\section{Appendix G: Tapeout Confirmation}

\section{VOBLAQ In Fab (Stability Detectors)}

The MOsIS Service <xmosis@mosis.com>

Wed, Jan 5, 2011 at 2:06 PM

To: clee83@calpoly.edu

MOSIS run VOBL containing design 84258 (Stability Detectors)

is now being fabricated. Your Fab-ID is VOBLAQ.

The Fab-ID uniquely identifies your project on its wafer lot

and consists of two parts: the run (VOBL) and the die (AQ).

For status information concerning your project, please visit the URL https://www.mosis.com/db/pubf/proj status?page type=login.

When the fabrication cycle is completed, MOSIS will ship your order

to you via FedEx 2nd Business Day service.

Project Status:

Design 84258 status: IN FAB

Design name: Stability Detectors

E-mail address: clee83@calpoly.edu

Phone number: $(805) 455-0690$

Technology: SCN3M_SUBM, lambda $=.3$

Fabrication restricted to AMI only.

Fill to be added: No

Fabricated on run VOBL (AMI_C5F) as VOBLAQ.

Bonding pads: 40

Layout size: $1490 \times 1490$ microns; area: 2.217 sq millimeters

Layers found (and densities): ACTIVE, CONTACT, GLASS, METAL1 (26.1\%), METAL2 (17.5\%), METAL3, N_PLUS_SELECT, N_WELL, POLY (1.3\%), P_PLUS_SELECT, P_WELL, VIA, VIA2

Requested quantity: 40

Requested packaging: DIP40 [MOSIS generated bonding diagram]

(10 parts), UNPACKAGED (30 parts)

Maximum die size: $2250 \times 2250$

VOBL is in fabrication; parts are expected at MOSIS on

30-MAR-2011 (MOSIS projected).

PO: V0000916

A total of 40 parts are ordered

with 10 to be packaged in DIP40

with 30 to be delivered unpackaged

The charge for project fabrication will be $\$ 230$ per the MOSIS

quote \#159500-A.

plus $\$ 20.13$ in sales tax

The shipping address of record is:

CIO CAMPUS DINING RECEIVING DOCK

Cal Poly State University

One Grand Ave

BLDG 19

San Luis Obispo, CA 93407-0466

Mosis-Reply-Id: 00371384-001-006 


\section{Appendix H: Initial Bonding Diagram}

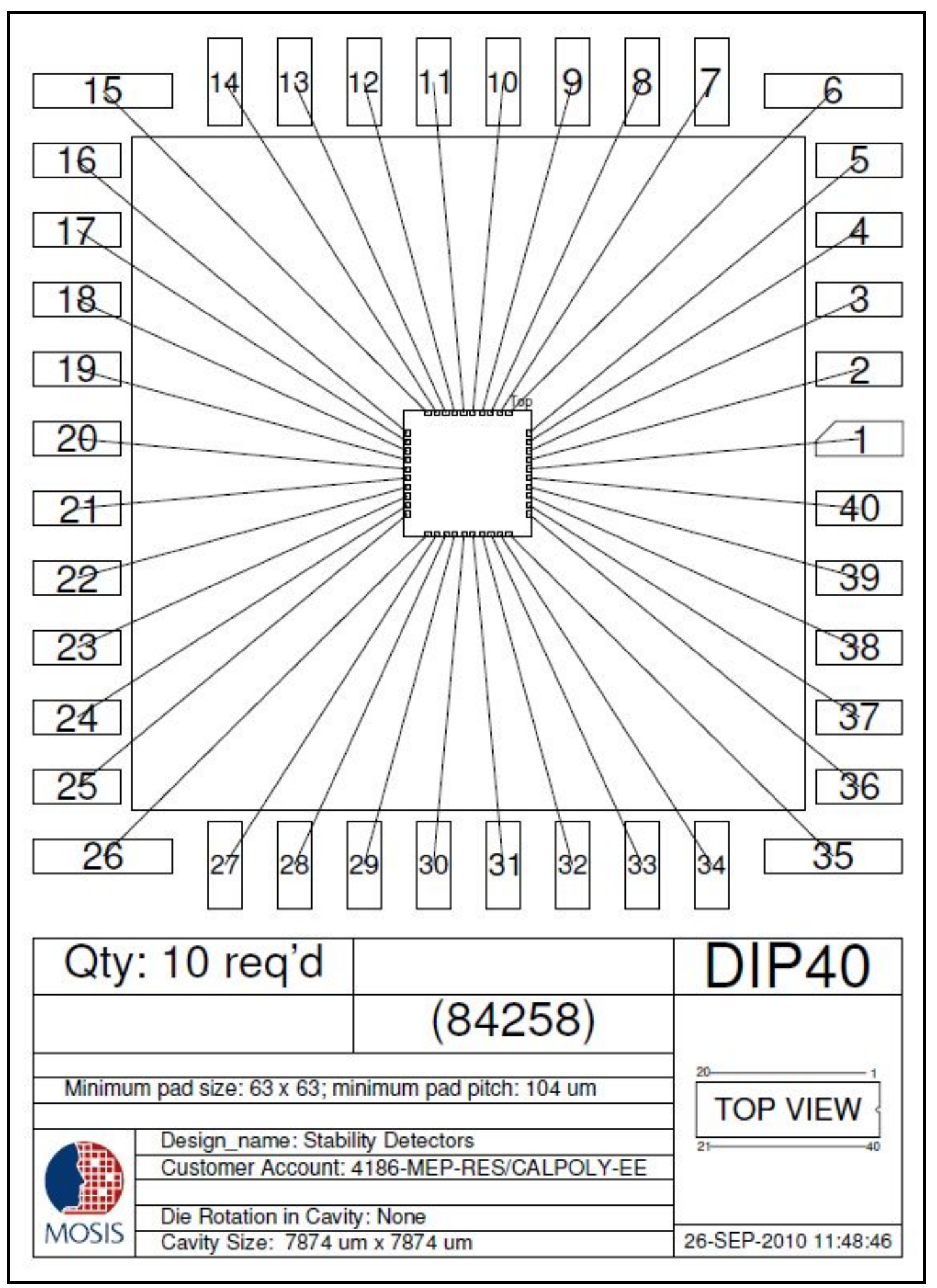




\section{Appendix I: Notification of Fabrication Failure}

\section{VOBL ON-SEMI C5 schedule delay notification}

The MosIS Service<amosis@mosis.com>

Thu, May 5, 2011 at 4:15 PM

Blind-Carbon-Copy

To: noreply-support@mosis.com

Subject: VOBL ON-SEMI C5 schedule delay notification

From: support@mosis.com (MOSIS Customer Support)

Reply-To: support@mosis.com (MOSIS Customer Support)

Date: Thu, 05 May 2011 16:15:01 -0700

Sender: amosis@mosis.com

Dear Designers,

We regret that the foundry has reported the initial VOBL ON-SEMI C5

fabrication lot has failed due to high VIA2 resistance at final test.

Replacement wafers have been started and the foundry is expediting

fabrication and initial estimate is the parts will be avallable the

end of July.

You can monitor this status using the following web form.

https: $/ /$ www. mosis.com/db/pubf/cact?page type=login

Please send any questions or comments to suppert@mosis.com or the new MOSIS Support Inquiry System, it's very easy to create

a support account if you do not already have one:

https: //www.mosis.com/db/pubf/cs?page_type=new_account

and once created, please log in using:

https://www.mosis.com/db/pubf/cs?page_type=login

$-\cdot$

MOSIS Support

End of Blind-Carbon-Copy 


\section{Appendix J: Notification of Packaging Anomaly}

\section{VOBL, Packaged parts die corrosion anomaly}

Robert Wormuth <wormuth@mosis.com>

Wed, Jul 6, 2011 at 1:43 PM

Reply-To: Robert Wormuth <wormuth@mosis.com>

Cc: support@isi.edu, wormuth@mosis.com

Dear Designers,

Our packaging vendor has reported a pad metal anomaly that may affect some packaged parts on the VOBL run. Unpackaged dice are not affected and are expected to ship to customers by July 6 .

Auger analysis has been performed on affected parts. No contaminating material was found, and the packager has concluded that the anomaly would not continue to spread on the pads. To test this conclusion, one of the packaging vendor's customers who was affected by the same processing disturbance packaged some parts and performed electrical tests.

All parts passed electrically. This customer then performed destructive bond pull tests, and all parts met MIL-STD- 883 specifications. In addition, this customer performed 96 hours of burn-in at $125 \mathrm{C}$ and electrically tested the parts again. All parts still passed.

Visual inspection of the units confirmed that there was no change in the appearance of the pads.

Our packaging vendor's customer concluded that all parts with this anomaly are acceptable in their critical application.

Our packaging vendor is taking corrective action to ensure that this will not happen again.

MOSIS has shipped or is in the process of shipping packaged parts with this anomaly. Can you please review and let us know if you have any concerns with any of the packaged parts that you receive? At your request MOSIS will provide replacement packaged parts with dice that were not affected by the processing disturbance described above.

MOSIS requests that you send your reply via our customer support interface at http://support.mosis.com/

This web-based system allows MOSIS customers and the general public to submit inquiries to MOSIS 24 hours a day for automatic distribution to an appropriate MOSIS staff member.

Thank you,

The MOSIS Service 


\section{Appendix K: Final Bonding Diagram}

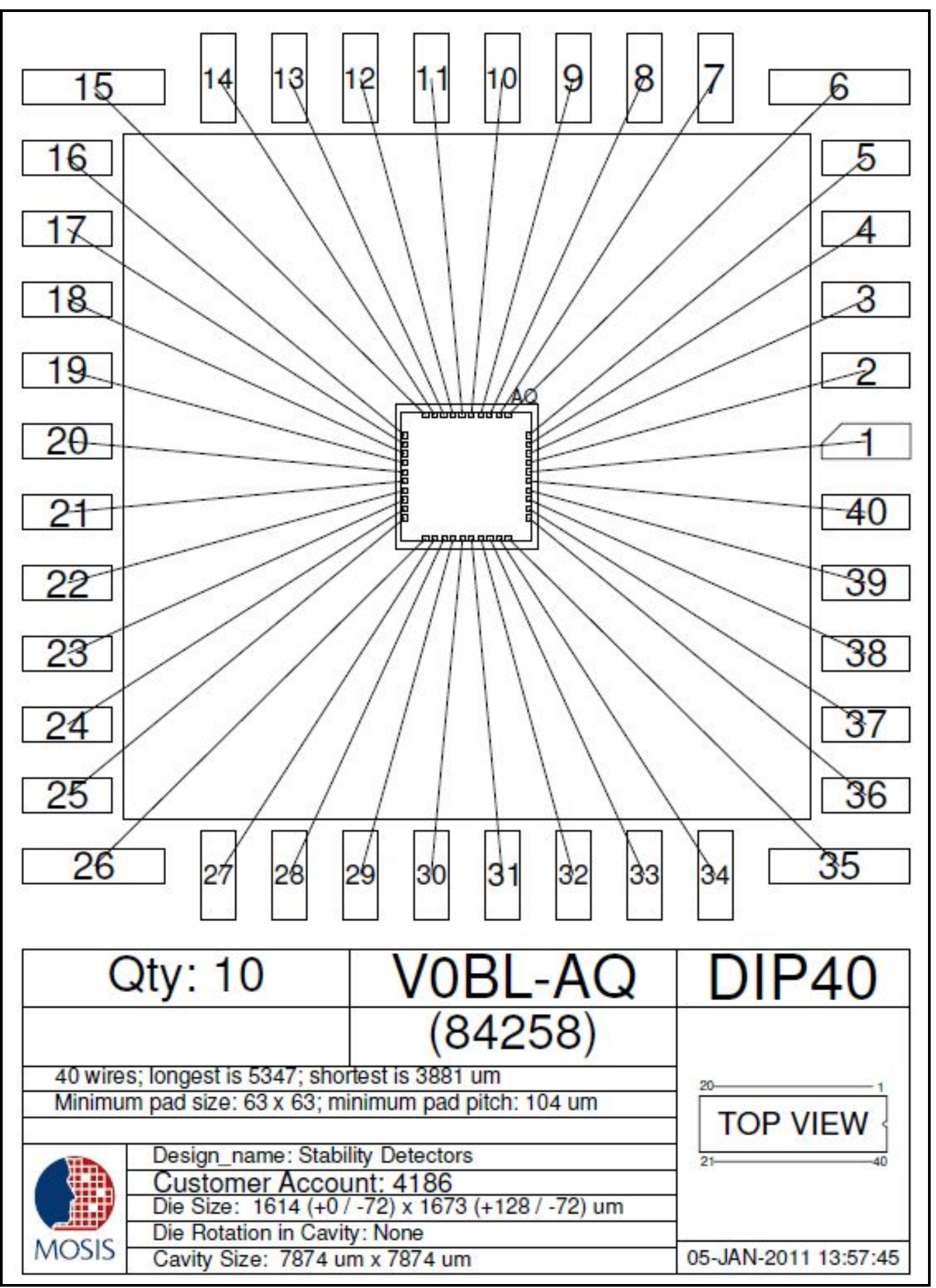




\section{Appendix L: Die Structure Measurements}

\section{Horizontal Dice Measurements}

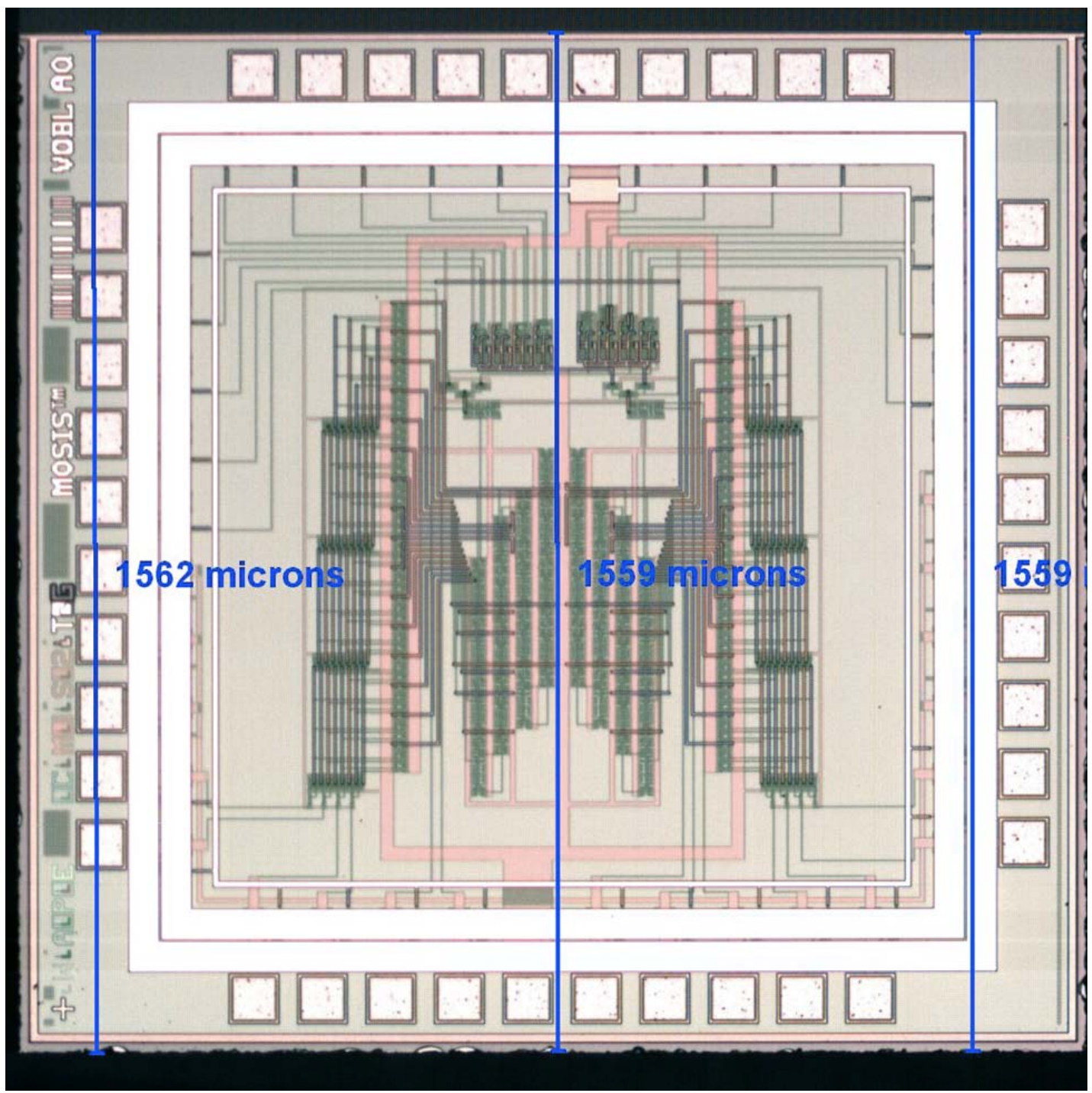




\section{Vertical Dice Measurements}

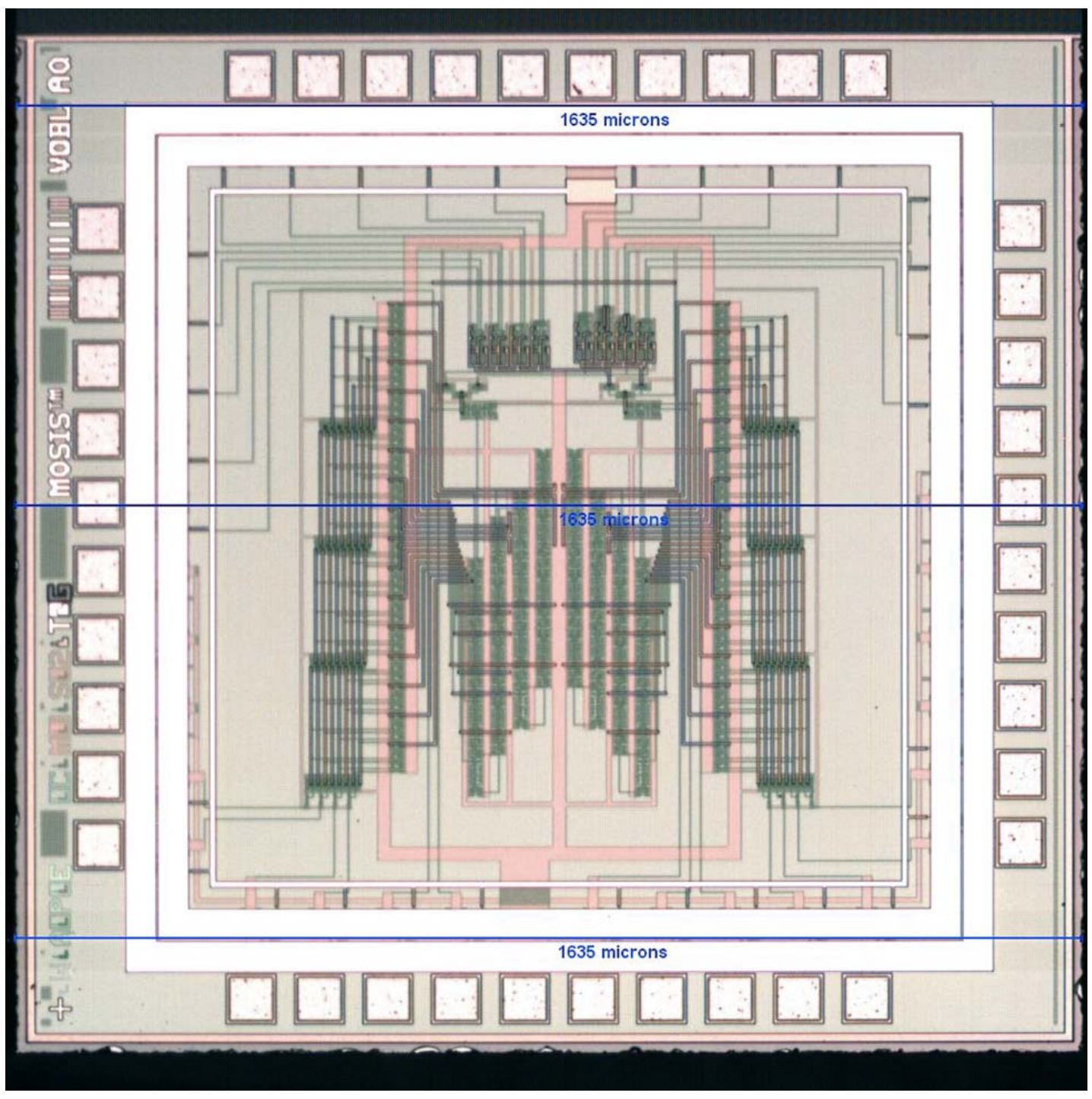


Measurements for Delay Chain and Decoder Modules

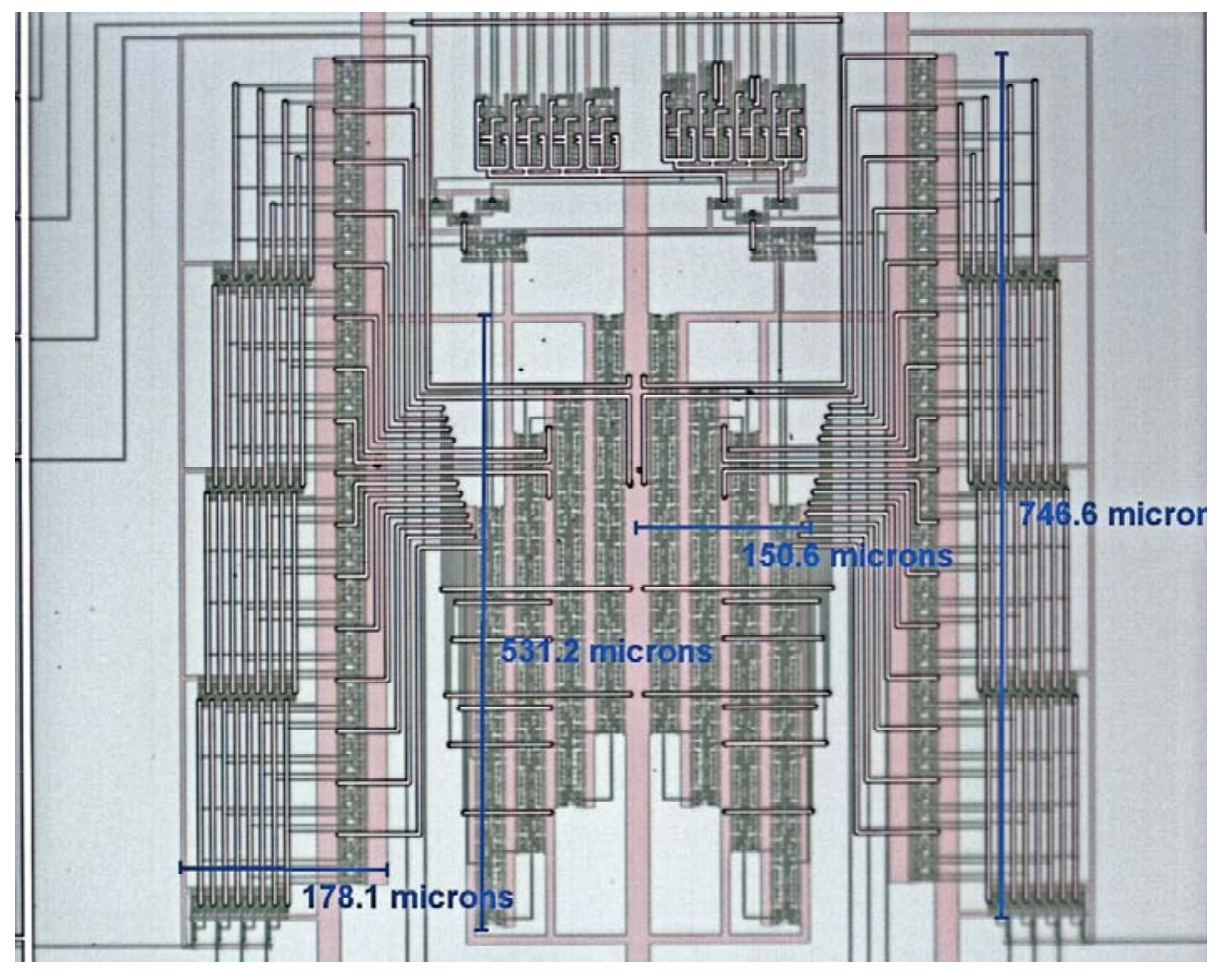

Measurements for Cell Heights

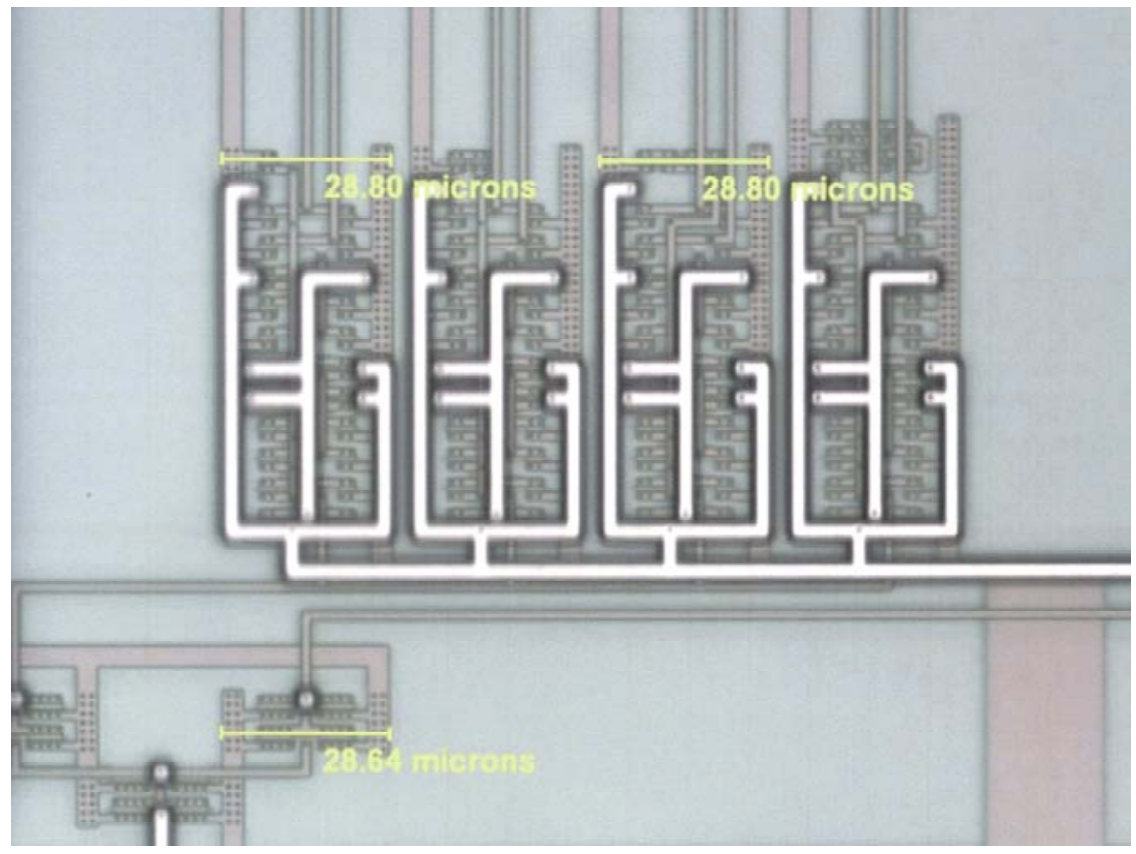




\section{Appendix M: Chip Pinout}

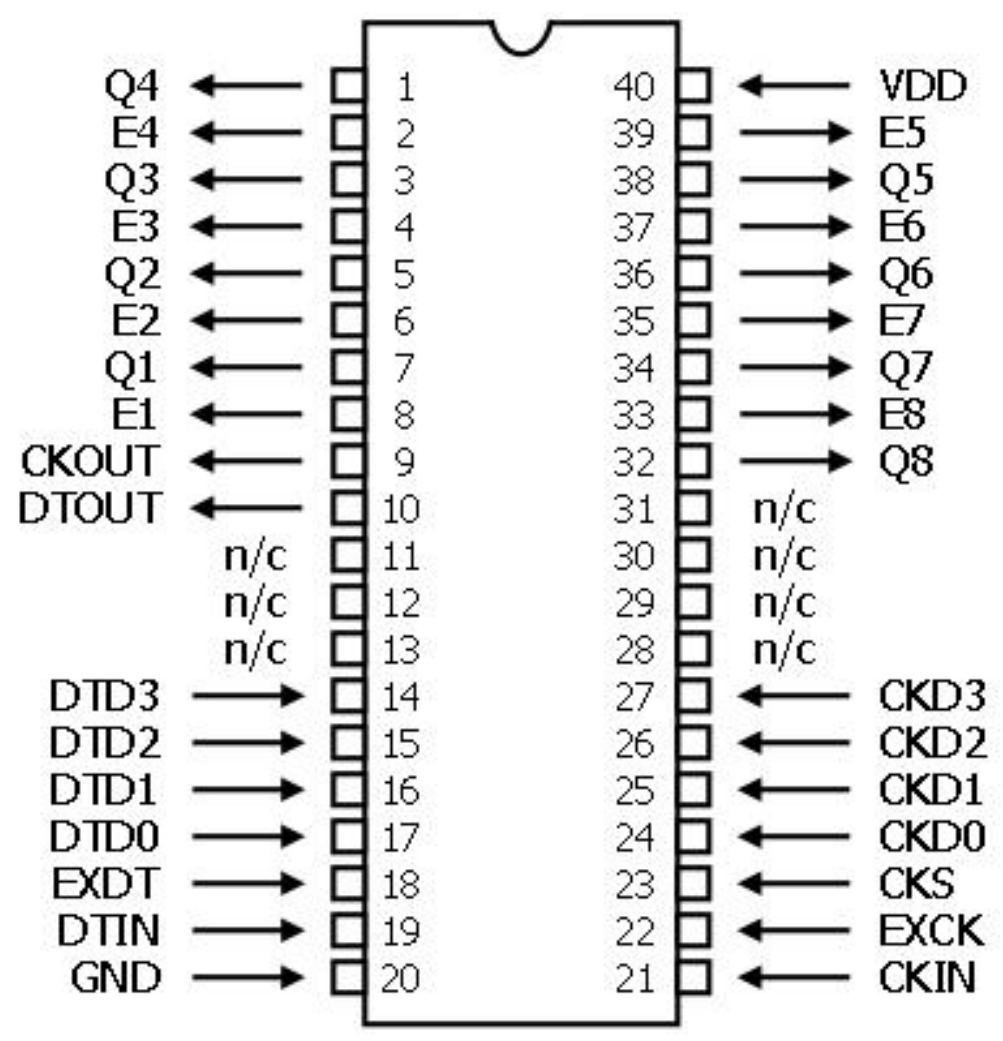




\section{Appendix N: SPICE Codes for Post-Silicon Verification}

\section{N.1 Configurable Delay Chain}

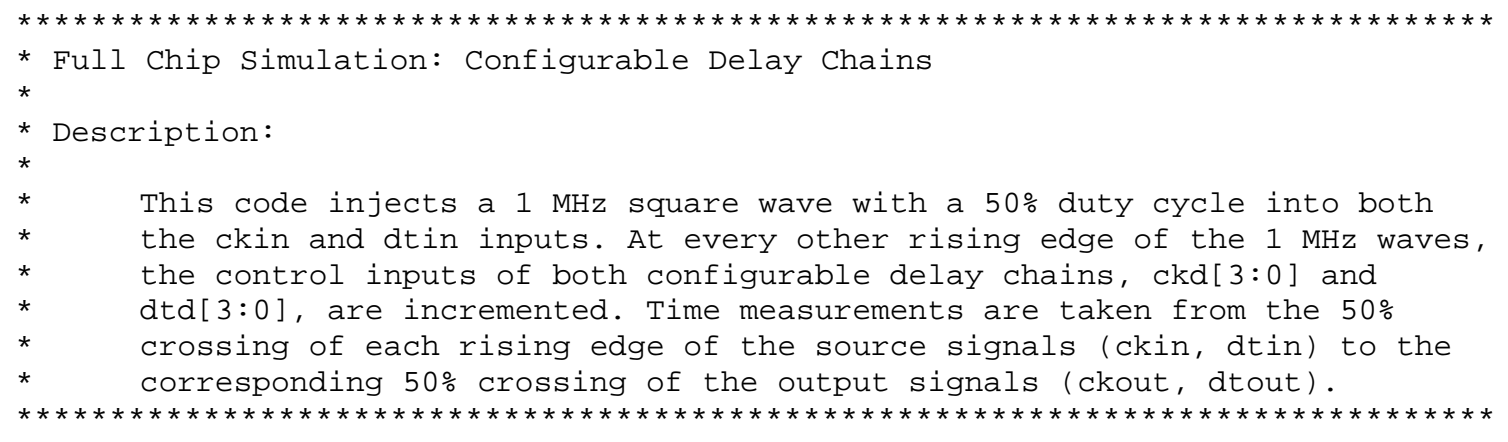

vdd vdd $\odot$ dc 5

vcks cks $\odot$ dc $\odot$

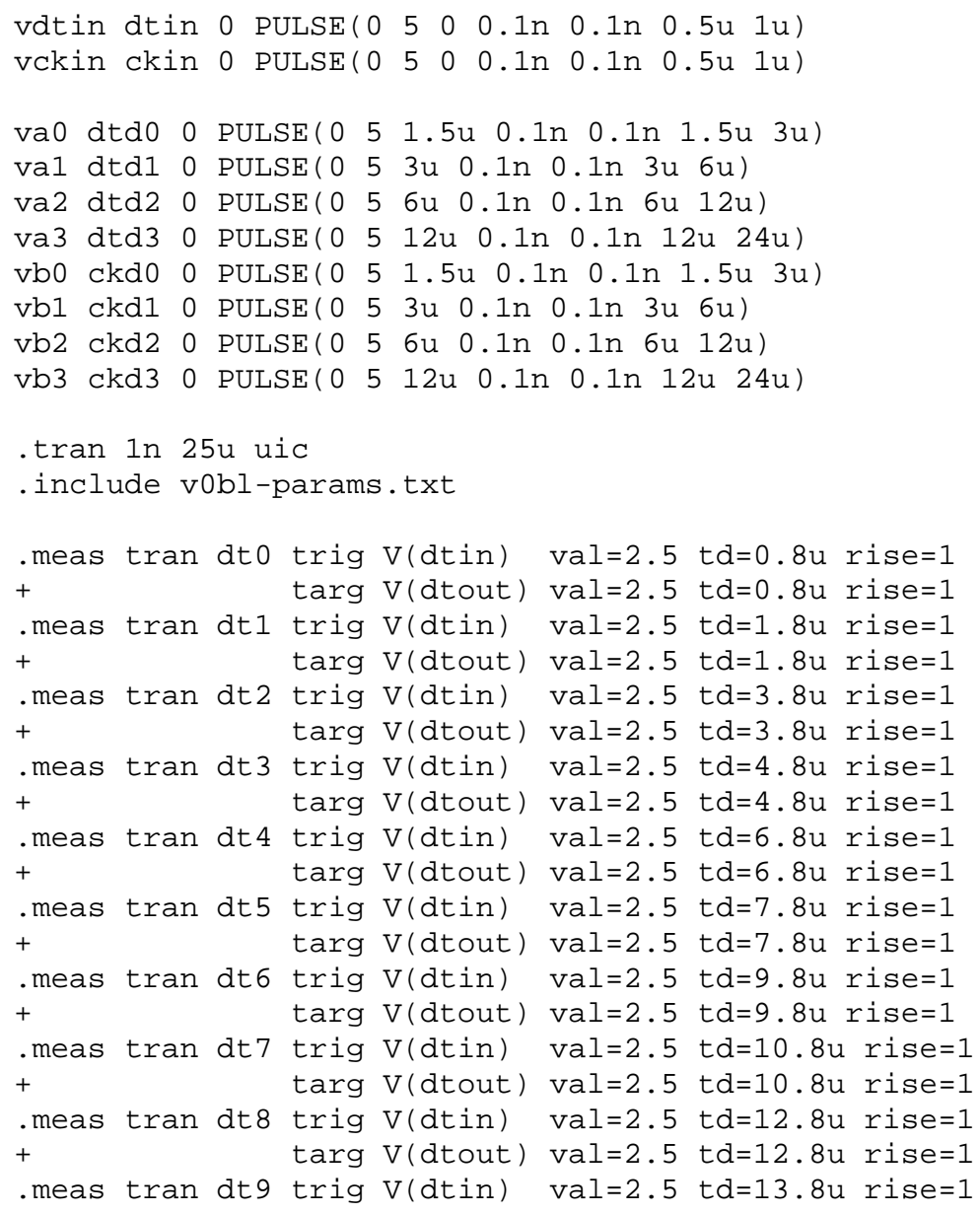




\begin{tabular}{|c|c|c|c|c|}
\hline & & & $\operatorname{targ} V($ dtout $)$ & val $=2.5$ td=13.8u ris \\
\hline meas & tran & $\mathrm{dt} 10$ & $\operatorname{trig} V(d t i n)$ & val $=2.5$ td $=15.8 u$ rise $=1$ \\
\hline & & & $\operatorname{targ} \mathrm{V}(\mathrm{dtout})$ & $t d=15.8 u$ rise $=1$ \\
\hline meas & tran & dt11 & trig $V(d t i n)$ & $t d=16.8 u$ \\
\hline & & & $\operatorname{targ} V($ dtout $)$ & $t d=16.8 u$ \\
\hline meas & tran & $\mathrm{dt} 12$ & rig V(dtin) & $\mathrm{val}=2.5 \mathrm{td}=18.8 \mathrm{ur}$ \\
\hline & & & $\operatorname{targ} V($ dtout $)$ & $\operatorname{val}=2.5 \mathrm{td}=18.8 \mathrm{u}$ rise \\
\hline meas & $\operatorname{tran}$ & dt13 & trig $V(d t i n)$ & $\mathrm{val}=2.5 \mathrm{td}=19.8 \mathrm{u} \mathrm{ri}$ \\
\hline & & & $\operatorname{targ} V($ dtout $)$ & val $=2.5$ td=19.8u ris \\
\hline meas & tran & dt14 & trig $V(d t i n)$ & $\operatorname{val}=2.5 \mathrm{td}=21.8 \mathrm{u} \mathrm{ri}$ \\
\hline & & & $\operatorname{targ} V($ dtout $)$ & val $=2.5 \mathrm{td}=21.8 \mathrm{u}$ ris \\
\hline meas & tran & dt15 & trig V(dtin) & $\mathrm{val}=2.5 \mathrm{td}=22.8 \mathrm{u}$ rise $=1$ \\
\hline & & & $\operatorname{targ} V($ dtout $)$ & $2.5 \mathrm{td}=22.8 \mathrm{u}$ ris \\
\hline meas & tran & ckO & tr & $.8 u$ rise $=1$ \\
\hline & & & $\operatorname{targ} \mathrm{V}(\mathrm{c}$ & val $=2.5 \mathrm{td}=0.8 \mathrm{u}$ rise $=1$ \\
\hline meas & $\operatorname{tran}$ & ck1 & $\operatorname{trig} \mathrm{V}(\mathrm{c}$ & $2.5 \mathrm{td}=1.8 \mathrm{u} r \mathrm{i}$ \\
\hline+ & & & targ & $=1.8 u$ \\
\hline . meas & tran & ck2 & $\operatorname{trig} \mathrm{V}(\mathrm{c}$ & $=3.8 \mathrm{u}$ ris \\
\hline+ & & & $\operatorname{targ} \mathrm{V}(\mathrm{c}$ & val $=2.5 \mathrm{td}=3.8 \mathrm{u}$ ris \\
\hline meas & $\operatorname{tran}$ & ck3 & $\operatorname{trig} \mathrm{V}(\mathrm{c}$ & $\mathrm{val}=2.5 \mathrm{td}=4.8 \mathrm{u} \mathrm{ri}$ \\
\hline- & & & ta & $\operatorname{val}=2.5 \mathrm{td}=$ \\
\hline meas & tran & ck4 & trig & $2.5 \mathrm{tc}$ \\
\hline+ & & & targ & $.8 u$ ri \\
\hline meas & tran & ck5 & trig & val=2.5 td=7.8u ris \\
\hline+ & & & $\operatorname{targ} \mathrm{V}(\mathrm{c}$ & $\mathrm{val}=2.5 \mathrm{td}=7.8 \mathrm{u}$ \\
\hline meas & tran & ck6 & trig Vi & $\mathrm{val}=2.5 \mathrm{tc}$ \\
\hline+ & & & targ & $.8 u r i$ \\
\hline meas & $\operatorname{tran}$ & ck7 & trig & $0.8 \mathrm{u} \mathrm{ri}$ \\
\hline+ & & & targ & $\operatorname{val}=2.5 \mathrm{td}$ \\
\hline meas & tran & ck8 & trig $\mathrm{V}(\mathrm{c}$ & $\mathrm{val}=2.5 \mathrm{td}=12.8 \mathrm{u}$ \\
\hline+ & & & $\operatorname{targ} \mathrm{V}(\mathrm{c}$ & $\operatorname{val}=2.5 \mathrm{td}$ \\
\hline . meas & $\operatorname{tran}$ & ck9 & trig $\mathrm{V}(\mathrm{c}$ & $13.8 u$ rise $=1$ \\
\hline+ & & & $\operatorname{targ} \mathrm{V}(\mathrm{cl}$ & $3.8 \mathrm{u}$ rise $=1$ \\
\hline me & $\operatorname{tran}$ & ck10 & trig $V(c$ & $=15.8 u$ rise $=1$ \\
\hline & & & $\operatorname{targ} \mathrm{V}($ & $t d=15.8 u$ rise $=1$ \\
\hline meas & tran & ck11 & rig Vi & $\mathrm{td}=16.8 \mathrm{u}$ \\
\hline+ & & & $\operatorname{targ} \mathrm{V}(\mathrm{c}$ & rise $=1$ \\
\hline . meas & $\operatorname{tran}$ & ck12 & $\operatorname{trig} \mathrm{V}(\mathrm{c}$ & $\mathrm{td}=18.8 \mathrm{u}$ rise $=1$ \\
\hline+ & & & $\operatorname{targ} \mathrm{V}(\mathrm{c}$ & td $=18.8 u$ rise $=1$ \\
\hline . me & tran & ck13 & trig $v(c$ & td $=19.8 u$ rise $=1$ \\
\hline+ & & & $\operatorname{targ} v(c$ & $t d=19.8 u$ \\
\hline & $\operatorname{tran}$ & ck14 & $\operatorname{trig} \mathrm{V}(\mathrm{c}$ & $t d=21.8 u$ \\
\hline+ & & & $\operatorname{targ} \mathrm{V}$ (ckout) & $t d=21.8 u$ \\
\hline$\cdot \mathrm{m}$ & $\operatorname{tran}$ & kc15 & $\operatorname{trig} \mathrm{V}(\mathrm{ckin})$ & $t d=22.8 u$ \\
\hline & & & $\operatorname{targ} V(c$ & $\mathrm{val}=2.5 \mathrm{td}=22.8 \mathrm{u}$ rise $=1$ \\
\hline
\end{tabular}




\section{N.2 Stability Checker Glitch Detection}

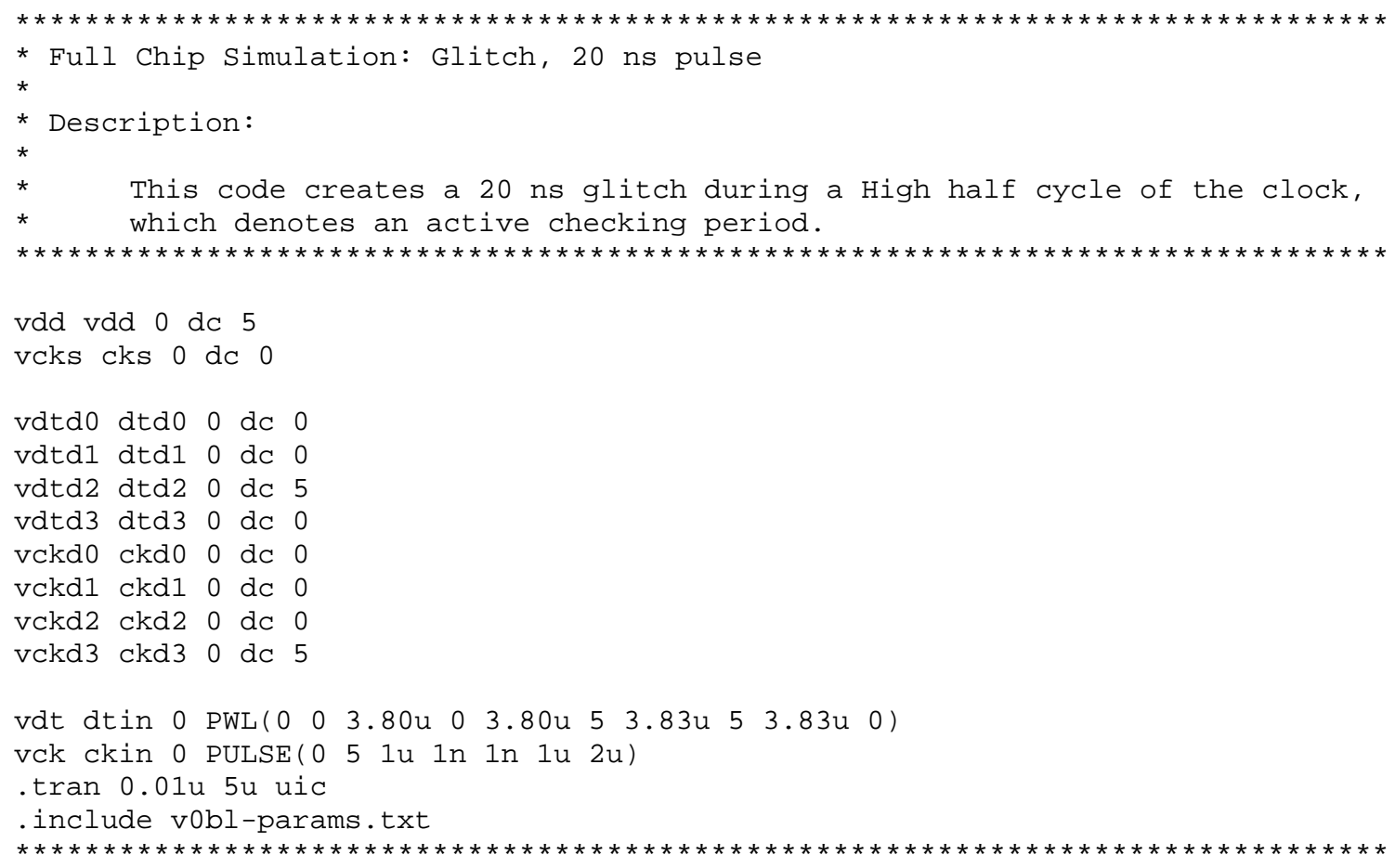

\section{N.3 Stability Checker Late Signal Detection}

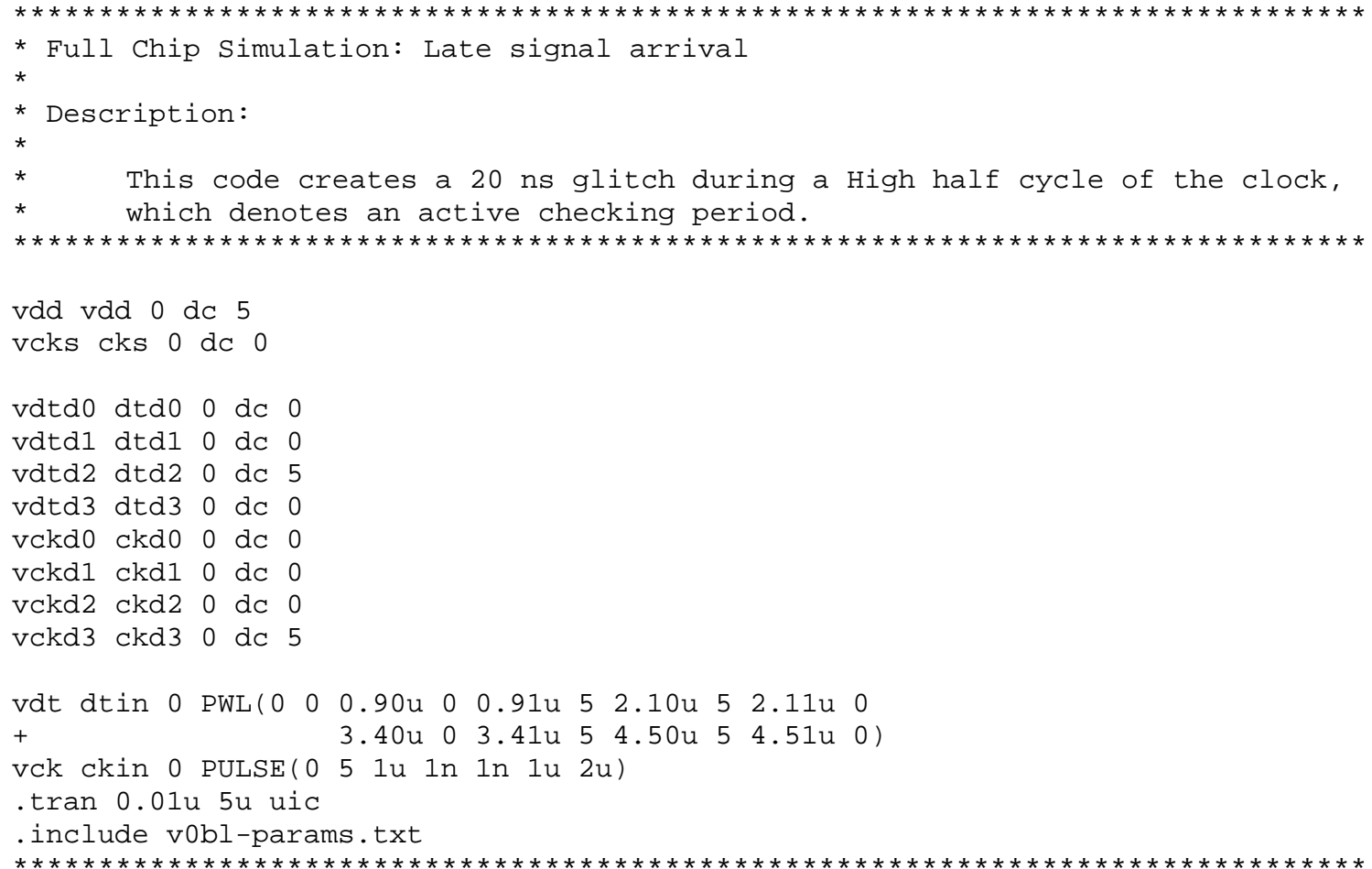




\section{Appendix O: MOSIS Educational Program}

This section describes the MOSIS accounts types, educational programs, account application procedures and differences between the MEP Research and Instructional Accounts

\section{O.1 Accounts for Academic Institutions}

The three types of accounts available for academic customers are:

- Commercial Account

- MEP Instructional Program Account

- MEP Research Program Account

All accounts have a common application process and have to be requested by a faculty member. MOSIS does not charge a registration fee to open an MEP account.

\section{O.1.1 Commercial Account}

Commercial accounts differ from the other two academic accounts in that the cost of fabrication is entirely paid for by the customer. In contrast, other academic accounts qualify for partial or full grants and fabrication subsidies. The primary contact for a commercial account has to be either a professor or class instructor. 


\section{O.1.2 MEP Account}

The MOSIS service has been funding educational programs since 1986, jointly by the National Science Foundation (NSF) and the Defense Advanced Projects Research Agency (DARPA) [30]. In 2000, MOSIS initiated the MOSIS Educational Program and has since been the sole provider of funds for academic projects. MOSIS no longer receives funds from any government agencies but continues to support academic projects out of its revenue from commercial operations. The goal of the MEP is to "aid educational institutions in the development of instructional and research programs in integrated circuits within their engineering departments”. MEP program partners include:

- ON Semiconductor

- IBM Microelectronics

- Cadence Design Systems (Cadence University Program)

- Mentor Graphics (Higher Education Program)

The MEP consists of two programs, an instructional and research component. The Instructional Program was established to provide a means for organized classes in IC design to experience the entire process of design, fabrication and testing. On the other hand, the Research Program caters to unfunded research conducted by graduate students and professors who "needed help developing critical mass in their areas of research in order to attract funding for future research".

Instructional Program accounts provide receive free fabrications for student designs in organized classes associated with accredited universities. The classes have to be graded, have a curriculum and finishes at the end of the semester. ON Semiconductor, as one of the sponsoring vendors, subsidizes two fabrication runs per academic year for 
the C5 $0.5 \mu \mathrm{m}$ process. The application process for a MOSIS Instructional Account includes a commercial agreement, description of the course, background of the teaching professor and a vendor document access application. This application also serves as a request for a grant from MOSIS to cover fabrication costs for the course.

Research Program accounts were initially sponsored by MOSIS. However, due to the growing number of research projects over the years and several cases of abuse [31], MOSIS limits fabrication subsidy to the cost of one chip per university campus. The subsidized chip should have an area not exceeding 16 square millimeters. Additional details about the MOSIS Research Program are described in the next section.

\section{O.2 MEP Research Program}

As described earlier, Research Accounts are meant for projects that result in some form of academic publication. Academic customers in the Research Program are expected to cover not only the cost of chip fabrication (excluding 1 subsidized chip), but also the entire cost of packaging. There are no restrictions on the number of chips fabricated or area used. The MOSIS subsidy was approved with an endorsement letter from the university, included in Appendix A.

Fabrication of the free chip is provided on a "space available basis" on multiproject wafers (MPW). As it is, MOSIS does not guarantee fabrication on the schedule selected by the customer but instead relies on production capacity at the partner foundries as well as other project orders on the shared MPW. 
The entire process of initiating a Research Account up to tapeout can be broken down into 4 phases:

- Setting up a MOSIS Customer Account

- Accessing Vendor Documents

- Submitting a Purchase Order to MOSIS

- Submitting a Design to MOSIS (Tapeout)

\section{O.2.1 Setting Up A MOSIS Customer Account}

The application process for a Research Account is similar to that of a Commercial Account. The 2 items in the application package are:

- Commercial Account Application - registers a primary contact, who has to be a class professor or instructor

- MOSIS Customer Agreement - acknowledgement by customer on MOSIS' policies regarding the fabrication process e.g. MOSIS does not guarantee that fabricated parts will be functional, fabrication schedule, etc.

\section{O.2.2 Accessing Vendor Documents}

The next step is to obtain process technology-specific information for circuit and layout design. Partnering vendors sub-license MOSIS to distribute proprietary documents containing design rules, process specifications and SPICE parameters. These documents are useful as design references and for circuit simulation. 
The application to access these proprietary documents differs from one vendor to another. For accounts associated with ON Semiconductor, the customer is required to submit 3 other documents in addition to the initial application described in 5.3.1. These documents require an accompanying signatory from the university's legal department to acknowledge the authority of the professor to enter into legal agreement. The main aspects in those documents are briefly summarized as follows [32]:

- Confidentiality Agreement (CDA)

o Customer agrees not to use any of the proprietary information other than for the purpose of the business relationship (relating to circuit design for manufacturing) with MOSIS

o Customer agrees to not divulge any proprietary information to any third parties.

- Design Kit License Agreement (DKLA)

o MOSIS grants customer a non-transferable license to use the provided technical data in the design kit

o Customer assumes the risk that modification in the technical data not performed at the direction of MOSIS or approved third party may result in designs that are incompatible with targeted manufacturing process 
- Academic Account Document Access Application

o Contains details on account information, fabrication plan, account liaison non-disclosure agreement (NDA), list of students and other faculty that have signed individual NDAs

o Mini-Proposal describing project details including requested fabrication process, chip dimensions, fabrication and packaging plans and test plans.

\section{O.2.3 Submitting a Purchase Order to MOSIS}

The purchase order is issued to a vendor to authorize the expenditure of funds for goods and services. It covers all process expenses (fabrication and packaging), shipping costs and tax. Purchase orders are invoiced $80 \%$ at tapeout and $20 \%$ when completed parts are shipped back to the customer. The amount invoiced at tapeout covers a data preparation fee and phototooling charge for IC fabrication while the amount invoiced after shipping covers packaging costs. The total manufacturing costs for this project was \$2920. The price quotation is included in Appendix B.

\section{O.2.4 Submitting a Design to MOSIS}

Customers are provided with an online account on the MOSIS website after the initial application process is approved. Aside from storing contact details and legal information, the online customer account is used to create projects for fabrication, and also to download vendor specific documents. MOSIS provides a flow chart (Fig. O.2.1) that guides customers through the design stages through to tapeout. The process starts 
with a new project request and ends with taping out verified design files via FTP for a manufacturability review.

Submit A Design To MOSIS Chart

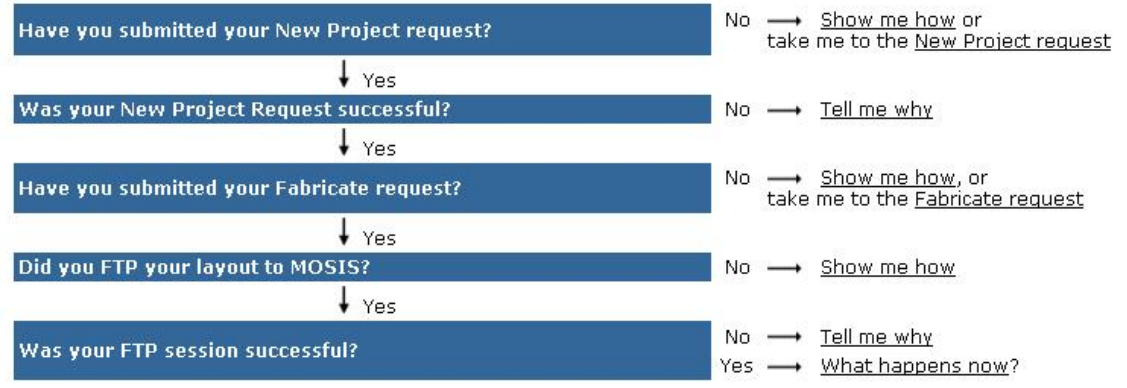

\begin{tabular}{|c|c|c|}
\hline Do you need to update information about your project? & Yes & $\overrightarrow{\text { take }}$ me to the Undate Request Form \\
\hline Do you need to send new layout? & Yes & 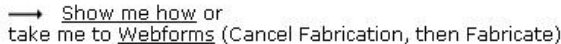 \\
\hline Can I practice submitting a design? & Yes & $\longrightarrow$ Tell me more \\
\hline
\end{tabular}

Fig. O.2.1 MOSIS Design Submission Flow[35] 